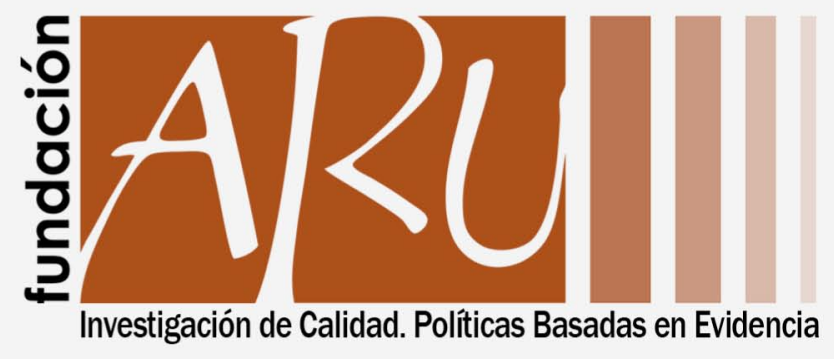

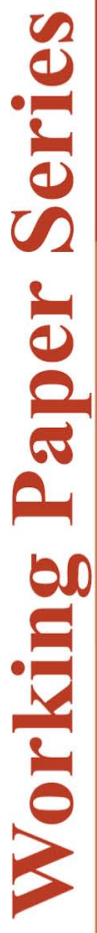

Efectos intencionados y no intencionados de transferencias monetarias no condicionadas a los adultos mayores: El caso de la Renta Dignidad

Werner L. Hernani-Limarino

Gary Mena 


\title{
Efectos intencionados y no intencionados de transferencias monetarias no condicionadas a los adultos mayores: El caso de la Renta Dignidad
}

\author{
Intended and unintended effects of unconditional cash \\ transfers: The case of Bolivia's Renta Dignidad
}

\author{
Werner Hernani-Limarino \\ Gary Mena \\ Marzo 2015 \\ Resumen $^{1}$
}

Este documento presenta una evaluación de impacto cuasi-experimental de los efectos intencionados y no intencionados de la Renta Dignidad, una pensión universal no contributiva de vejez en Bolivia, sobre variables de bienestar, ahorro, e inversión del hogar y resultados del mercado laboral de beneficiarios directos e indirectos. Aprovechamos un cambio en política exógeno que reduce la edad para ser beneficiario de 65 a 60 años para identificar los efectos causales del programa. Nuestra estrategia de identificación se base en los modelos de Diferencias-en-Diferencias y Cambios-en-Cambios para calcular no solo el efecto promedio sino también en cuantiles específicos. Encontramos que la pensión no contributiva tiene complejos efectos intencionados y no intencionados en diferentes resultados individuales y del hogar sobre diferentes tipos de individuos y hogares. En el caso de las mujeres encontramos que el programa ha incrementado en promedio el ingreso no laboral de sus hogares, lo que ha reducido su oferta laboral e ingreso laboral, lo que a su vez ha disminuido el ingreso laboral de sus hogares. En el caso de los hombres, la transferencia ha incrementado significativamente el ingreso no laboral de sus hogares, pero el incremento en el el ingreso del hogar per-cápita no es significativo.

Palabras clave: Evaluación de impacto, transferencias monetarias no condicionadas, sistema de protección social no contributiva, programas para adultos mayores

\begin{abstract}
This document presents a quasi-experimental impact evaluation of Renta Dignidad, a universal non-contributory pension to old-age population in Bolivia, on intended and unintended outcomes including: household's welfare, investments and savings, and labor market outcomes of direct and indirect beneficiaries. To identify the causal effects of the

\footnotetext{
${ }^{1}$ Los hallazgos y conclusiones presentadas en este documento son de los autores y no necesariamente representan los puntos de vista de la Fundación ARU ni de ninguna otra institución a la que se encuentren afiliados. Quisiéramos agradecer a los participantes del seminario desarrollado en Brasilia-Brasil en Septiembre de 2014 llamando "Social Protection, Entrepreneurship and Labour Market Activation" por los valiosos comentarios.
} 
program we take advantage of an exogenous policy change that reduces the eligibility criteria from 65 to 60 years in December of 2007. Our identifaction strategy relies on both, the simple Difference-in-Difference and the more complex Changes-in-Changes approach to calculate not only average, but also quantile treatment effects. We find that non-contributory household transfers have complex intended and unintended effects on different individual and household outcomes for different types of individuals and households. In the case of women, we find that non-contributory pensions have, on average, increased their households' non-labor income, which has decreased their labor supply and labor earnings, which in turn has decreased households' labor income. In the case of men, the transfer has increased their households' non-labor income and increased their households' per-capita income, but the latter is not significant.

Keywords: Impact evaluation, unconditional cash transfers, non contributive social protection system, elderly programs

Clasificación/Classification (JEL): C21, H2, H5, I3 


\section{Introducción}

Las pensiones no contributivas son usualmente justificadas como medio para proveer asistencia social a quienes no tienen acceso a un esquema de pensión contributiva. En el caso de Bolivia, estos son números grandes. La figura 1 presenta la proporción de la población en edad de trabajar que, al momento de ser entrevistada, se encuentra en el sector formal asalariado, o sea, uno que paga por seguridad social de corto (salud) y largo plazo (pensiones); y la proporción de la población adulta mayor que tiene acceso a una pensión contributiva. El porcentaje de hombres en el sector formal y que por ende contribuye a una AFP (esquema de pensión contributiva) durante 1999-2013 es menor al 17\% y en el caso de las mujeres es menor al 10\%. Incluso durante 2006-2013, un periodo que se caracteriza por una mayor actividad económica y una serie de reformas, el porcentaje de hombres afiliados a una pensión contributiva es $18.5 \%$ y el porcentaje de mujeres es $11.1 \%$. Más aun, es posible que el porcentaje de personas que van a tener acceso a una pensión contributiva al llegar a la tercera edad sea menor, a causa de que las personas transitan de ida y vuelta desde y hacia la informalidad o trabajos por cuenta propia (ver Hernani-Limarino y Eid, 2012). De hecho, en 2013 sólo $11.44 \%$ de los hombres y $8.03 \%$ de las mujeres en el cohorte de edad entre 60 y 65 años recibe una pensión contributiva (jubilación).

Esta situación no es diferente en muchos otros países de Latinoamérica y el Caribe, incluyendo Honduras, República Dominicana, El Salvador, Guatemala, Paraguay y Nicaragua, que cuentan con una cobertura de una pensión contributiva por debajo del $20 \%$, e incluso en Colombia y Perú, los niveles sólo llegan al 23\% y 25\%, respectivamente (Rofman y Oliveri, 2011). La baja cobertura de pensiones contributivas allanó el camino para la introducción de esquemas de pensiones no contributivas, como el Beneficio de Prestação Continuada en Brasil, 70 y Más en México, Pensión Básica Solidaria (ex PASIS) en Chile y las descritas en este documento para Bolivia.

Sin embargo, como nuestro título sugiere, la introducción de las pensiones no contributivas no sólo tendría efectos intencionados como el aumento de la renta disponible de los individuos y los hogares y por lo tanto aumentar su consumo e incluso sus inversiones en capital físico (por ejemplo, la mejora de su vivienda o compra de bienes duraderos) y en su capital humano propio o de sus compañeros de casa (salud y educación). Según la teoría, en función del monto de la transferencia no contributiva también puede reducirse la oferta de trabajo -por el efecto ingreso de una transferencia no condicional del gobierno, por el retiro prematuro de la fuerza de trabajo, cambios hacia sectores de empleo que no pueden proporcionar las pensiones contributivas o simplemente por la reducción de las horas de trabajo. Es ante la posibilidad de que surjan estos efectos secundarios que no solamente analizamos los impactos de la pensión no contributiva sobre el ingreso y consumo, sino también sobre las inversiones y el ahorro del hogar y sobre indicadores del mercado laboral.

En el caso de Bolivia, este no es el primer trabajo en investigar los efectos de las pensiones no contributivas en general y de la Renta Dignidad en particular. Martínez (2004) utiliza datos de encuestas de hogares de 1999 a 2002 y un diseño de regresión discontinua para concluir que las pensiones no contributivas tuvieron efectos positivos y estadísticamente significativos sobre el consumo, sobre todo el consumo de alimentos y las inversiones productivas. Yanez (2008) estudia el efecto de las pensiones no contributivas en inversiones en capital humano de los niños y encuentra que las mujeres son más eficaces en la promoción de inversiones en capital humano. Escobar et al. (2013) presentan la evaluación de impacto oficial del gobierno 
de la Renta Dignidad, basada en un diseño de regresión discontinua y en una muestra de 1,258 hogares en el intervalo de edad [55,65] obtenida a través de la Encuesta a Hogares con Personas Adultas Mayores (EPAM). Ellos encuentran que la Renta Dignidad no sólo aumenta el ingreso y el consumo de los hogares per-cápita, sino que también reduce la incidencia de la pobreza entre 11 y 14 puntos porcentuales alrededor del corte (60 años). También encuentran que estos efectos pueden atribuirse más a las zonas urbanas que a las rurales, por ejemplo, el impacto estimado sobre la reducción de la pobreza alrededor del corte fue de 16 a 19 puntos porcentuales en las áreas urbanas (estadísticamente significativo al nivel del 95\% de confianza), mientras que la magnitud varía entre 2 y 5 puntos porcentuales en las zonas rurales.

Claure y Hernani-Limarino (2014) utilizan los mismos datos y métodos para replicar la evaluación oficial y llevar a cabo algunas pruebas de falsificación. Ellos argumentan que la estrategia de estimación adecuada no es un diseño de regresión discontinua clara sino una difusa. Sus resultados muestran efectos sobre el ingreso y el consumo de los hogares percápita que son negativos y estadísticamente significativos con un nivel de confianza del $99 \%$ en las áreas rurales. Más aun, encuentran que tanto la dirección como el nivel de significancia de las estimaciones del tratamiento dependen mucho de la inclusión de un conjunto particular de controles. Por otra parte, las pruebas de falsificación revelan que hay saltos significativas en otros indicadores a lo largo de la distribución de edad. Esto sugiere la existencia de problemas, ya sea en la calidad de los datos o con el tamaño de la muestra, para aplicar una estrategia de regresión discontinua2. Es importante tener en cuenta que cualquier enfoque de discontinuidad no sólo se basa en la comparación y el poder de la muestra en torno al punto de corte, sino también en el supuesto de que el grupo de control no espera cruzar el punto de corte, o que por lo menos esta expectativa no afecta su comportamiento. Es decir, la población que no recibe la pensión no contributiva pero pronto la recibirá no se comporta como si ya la recibiera debido a la expectativa.

En este trabajo seguimos un conjunto diferente y, con suerte, más plausible de supuestos, al menos desde nuestro punto de vista. En lugar de confiar en la comparabilidad y el poder de la muestra en torno al corte de edad, como en el enfoque de regresión discontinua, y la comparabilidad de los grupos de tratamiento y de control condicionada en observables, como en toda identificación bajo el enfoque de ausencia de factores de confusión, aprovechamos el cambio exógeno de política que reduce el criterio de elegibilidad de la transferencia de 65 a 60 años. Utilizamos tanto el enfoque simple de Diferencias-en-Diferencias así como el enfoque más complejo de Cambios-en-Cambios (Athey e Imbens, 2006) para calcular los efectos no sólo promedio, sino también los efectos de tratamiento en cuantiles de la distribución del cohorte que fue afectado por el cambio de política, es decir la población $[60,65)$, comparándola con el cohorte más cercano que fue excluido del esquema no contributivo de pensiones, es decir la población con edad en el intervalo [55,60). Para mejorar el poder de nuestras estimaciones combinamos la muestra de tres años de encuestas de sección cruzadas antes y después del cambio de política, es decir, las encuestas de hogares de

2 Hernani-Limarino y Villarroel (próximo) tratan de resolver los problemas de calidad y de una muestra pequeña de la EPAM con el uso de un conjunto de encuestas de hogares oficiales armonizadas, bajo una estrategia de identificación que combina el diseño de discontinuidad con ausencia de factores de confusión. 
2005, 2006 y 2007 para el período ex-ante y las encuestas de hogares de 2008, 2009 y 2011 para el período ex-post.

Analizamos un conjunto comprehensivo de efectos intencionados y no intencionados, incluyendo (1) el bienestar de los hogares, medido por los ingresos no laborales, laborales y el ingreso del hogar per-cápita y el consumo del hogar per-cápita, y el consumo alimentario y no alimentario; (2) las inversiones del hogar en vivienda, en adquisición de bienes duraderos, en educación, en salud y el nivel y tasas de ahorro de los hogares; (3) los efectos sobre el mercado de trabajo en los beneficiarios directos del programa; y (4) los efectos en el mercado laboral de los beneficiarios indirectos del programa, es decir, población de jóvenes y adultos residiendo con un anciano que es beneficiario del programa. También intentamos no sólo identificar el efecto en la muestra completa, sino también diferenciar los efectos sobre los resultados de mujeres y hombres. Al igual que en las evaluaciones anteriores, se debe tener cuidado al interpretar los resultados, ya que no son representativos de toda la población de edad avanzada, sino del cohorte analizado. En nuestro caso el cohorte $[60,65)$ es el afectado por el cambio de política. En el caso de los diseños de discontinuidad los resultados son válidos sólo alrededor de la edad que define los criterios de elegibilidad.

El resto del documento se organiza de la siguiente manera: en la sección 2 presentamos los arreglos institucionales de las pensiones no contributivas de vejez en Bolivia. En la sección 3 describimos los datos y métodos que usamos para identificar los efectos causales de interés de la Renta Dignidad. En la siguiente sección (4) analizamos los impactos del programa sobre indicadores del hogar e individuales sobre los beneficiarios directos, futuros e indirectos. Presentamos nuestras conclusiones y las implicaciones de política en la sección final.

\section{Pensiones de vejez no contributivas en Bolivia}

En Bolivia hubieron tres generaciones de pensiones no contributivas desde 1996: el BONOSOL, el BOLIVIDA y la Renta Dignidad3. El Bono Solidario, también llamado BONOSOL, fue establecido en 1996 después del proceso de privatización de las empresas públicas. E1 BONOSOL era una renta anual de Bs. 1,300 a las personas mayores de 65 años de edad. Su principal objetivo fue de transferir los beneficios esperados del proceso de capitalización a todos los ciudadanos bolivianos que vivían en el territorio nacional, aunque no era un esquema universal ya que solo los mayores de edad al 31 de diciembre de 1995 tenían el derecho a recibirlo. Inicialmente el BONOSOL debería haber durado hasta 2001, sin embargo los resultados del proceso de capitalización no fueron los esperados y el programa no fue sostenible porque el número de beneficiarios fue subestimado.

Después de las elecciones de 1997, el nuevo gobierno suspendió los pagos del BONOSOL y en 1998 lo reemplazaron por el BOLIVIDA. Para asegurar la sostenibilidad del nuevo esquema, solamente los mayores de 50 años al 31 de diciembre de 1995 tenían el derecho de recibir la transferencia. El BOLIVIDA redujo la pensión a Bs. 395 por año y los pagos de las gestiones de 1998 y 1999 se realizaron recién en 2001. El gobierno incrementó el valor del BOLVIDA a Bs. 420 para los pagos de los años de 2000 y 2001, los cuales se efectuaron

3 Además de la transferencia monetaria, cada esquema de pensiones otorga gastos funerarios. 
recién en 2002. Ello implica que los adultos mayores recibieron solo $60.8 \%$ del valor original del BONOSOL en 2001 y $64.6 \%$ en 2002.

El partido original que creó el BONOSOL ganó las elecciones de 2002 y restauró su nombre original. Esta vez los que tenían 21 años al 31 de diciembre de 1995 tenían el derecho de recibirlo una vez que cumplan 65 años. El gobierno incrementó la transferencia hasta Bs. 1,800 ( $\approx$ USD 248). Los pagos del BONOSOL estuvieron fijados hasta diciembre de 2007. Después de esa fecha la autoridad pertinente debería redefinir el valor de la transferencia cada cinco años, basado en los recursos disponibles y la mortalidad de los beneficiarios.

En 2007 el actual gobierno reemplazó el BONOSOL con la Renta Dignidad, una pensión de vejez no contributiva universal4. A diferencia de los esquemas previos, los beneficiarios pueden recibir pagos de forma mensual, trimestral o anual5; dependiendo de sus necesidades y preferencias. Este nuevo esquema incrementó la transferencia hasta Bs. 2,400 para aquellas personas que no tienen derecho a una pensión contributiva y para quienes sí tienen acceso la transferencia es de Bs. 1,800 (75\%). Para asegurar el financiamiento se estableció una proporción fija del Impuesto Directo a los Hidrocarburos del 30\% en adición a los dividendos de las empresas públicas capitalizadas. El cambio más importante relacionado a nuestro estudio es la reducción en la edad que se requiere para recibir la transferencia de 65 a 60 años. Es ésta reducción en el corte de edad la variación exógena que utilizamos para identificar los efectos causales de la Renta Dignidad (tabla 1).

\section{Datos y métodos}

En esta sección presentamos no solamente los datos que utilizamos, sino también todas las fuentes de datos que podrían ser usadas para estudiar los efectos de la Renta Dignidad; justificando nuestra elección de las encuestas de hogares. También documentamos nuestra estrategia de identificación y describimos brevemente los procedimientos de estimación e inferencia que utilizamos.

\subsection{Fuentes de información disponibles}

Potencialmente hay tres fuentes de información que podrían usarse para analizar los impactos de la Renta Dignidad (tabla 2). Estas son:

Panel trimestral rotativo 2-2-2 de empleo o ETE.- Esta encuesta fue concebida para proveer información estadística sobre la oferta laboral y sus características, además de llenar el vacío entre las encuestas de hogares que son recolectadas anualmente. La encuesta se enfocó en el área urbana principalmente y es uno de los únicos casos de datos de panel en el país, ya

4 La constitución actual (2009) establece como derecho de todos los bolivianos mayores de edad que "[...] el estado proveerá una pensión de vejez, bajo el marco integral de un sistema de seguridad social." haciendo las pensiones no contributivas una obligación constitucional del estado.

5 Los pagos de la Renta Dignidad pueden acumularse por un periodo de hasta un año, de otra manera se pierde la transferencia de ese año. 
que las encuestas previas son datos de sección cruzada. Esta encuesta podría usarse para evaluar el impacto de la Renta Dignidad, en áreas urbanas, en indicadores del mercado laboral pero no seríamos capaces de estudiar las inversiones del hogar o el consumo. Se suponía que el panel dure desde 2009 hasta 2012 y que recoja la información de 8,532 hogares, pero el nivel de desgaste fue alto y la base de datos final no es pública.

La Encuesta de hogares con Personas Adultas Mayores (EPAM).- La EPAM es una encuesta de hogares que se llevó a cabo en 2011 y provee la información para la evaluación de impacto oficial, la cual se base en un diseño de regresión discontinua. La encuesta contiene información sobre las características demográficas, algunos indicadores del mercado laboral, el ingreso y consumo del hogar. Aunque la encuesta se enfoca principalmente en los adultos mayores y su estado socio-económico, los indicadores de consumo y del mercado laboral que se pueden construir no son tan completos como los de las encuestas de hogares. Para ilustrar el problema, la sección del mercado laboral no contiene información para generar una variable sobre el estado de formalidad de los trabajadores y la sección de consumo no es lo suficientemente comprehensiva como para asegurar la calidad del indicador de consumo.

Un detalle muy importante de la encuesta es que fue diseñada para ser comparable con la Encuesta Continua de Hogares 2003-2004 en términos del marco muestral. Si este fue el caso, entonces el área urbana habría sido sobre-muestreado como en la ECH 2003-2004, porque dicha encuesta se enfoca principalmente en recoger información de presupuestos familiares urbanos que sirvan para cambiar el año base del índice de precios al consumidor. Finalmente, como la encuesta se llevó a cabo solamente en 2011, las metodologías disponibles se reducen a aplicar un diseño de regresión discontinua, una aproximación ya usada en otros trabajos. 6

La serie armonizada de encuestas de hogares 1999-2013.- La principal fuente de microdatos en Bolivia son los datos de sección cruzada de encuestas de hogares del periodo 19992013. Éstas encuestas proveen información detallada sobre ingreso, consumo, mercado laboral, logros educativos, vivienda y características demográficas. Aunque el propósito principal de las encuestas de hogares no es para llevar a cabo evaluaciones de impacto, la tabla 2 muestra que sus tamaños de muestra en los intervalos de edad de interés son comparables a los de la EPAM, una encuesta específicamente diseñada para la evaluación de impacto de la Renta Dignidad usando un diseño de regresión discontinua. Mas aún, las encuestas de hogares que se han llevado a cabo son más ricas en términos de información desagregada.

Es por esto que decidimos usar la serie armonizada de encuestas de hogares para estimar los efectos intencionados y no intencionados de la Renta Dignidad sobre: i) el ingreso, ii) el consumo, iii) ahorros, iv) inversión en capital humano (educación y salud), v) adquisición de bienes durables, vi) inversiones en la vivienda; vii) participación en el mercado labora, vii) sector de empleo, ix) horas trabajadas y x) salario y remuneración (en los apéndices se encuentran las definiciones de las variables de interés).

6 Véase Escobar, Martínez y Mendizábal (2013) y Claure y Hernani-Limarino (2014). 


\subsection{Estrategia de identificación}

Para identificar los efectos causales de la Renta Dignidad usamos el modelo simple de Diferencias-en-Diferencias (DID) y su generalización conocida como Cambios-en-Cambios (CIC) propuesto por Athey e Imbens (2006). A continuación revisamos los supuestos básicos de identificación y analizamos si pueden aplicarse a nuestro problema.

\subsubsection{Set-up}

Empecemos con el modelo DID para el caso simple de dos periodos y dos grupos7. Sean los individuos indexados por el subíndice $i$ que pertenecen a uno de dos grupos $G_{i} \in\{0,1\}$, el grupo de control $G_{i}=0$ o al grupo de tratamiento $G_{i}=1$, y que son observados en dos periodos de tiempo $T_{i} \in\{0,1\} \quad$, el periodo ex-ante $T_{i}=0$, donde ninguno de los grupos son tratados, y el periodo ex-post $T_{i}=1$, en el cual el grupo de tratamiento recibe el tratamiento. Por lo tanto, podemos definir el indicador de tratamiento como sigue:

$$
I_{I}= \begin{cases}1 & \text { si } G_{I}=1 ; T_{i}=1 \\ 0 & \text { caso contrario }\end{cases}
$$

Bajo el supuesto de ausencia de factores de confusión compararíamos los cohortes de tratamiento ex-ante y ex-post, i.e. $(G=1, T=0)$ y $(G=1, T=1)$, o los cohortes de control y tratamiento ex-post $(G=1, T=1)$ y $(G=0, T=1)$. El enfoque de DID estándar nos sugiere en cambio comparar los grupos de control y tratamiento ex-post pero ajustando por las diferencias que observamos en la situación ex-ante. Sea el error aleatorio epsilon independiente de ambos $G$ y $T$, entonces el efecto de tratamiento se obtiene de acuerdo a:

$$
Y_{i}=\beta_{0}+\beta_{1} G_{i}+\beta_{2} T_{i}+\tau^{D I D_{I}+\varepsilon_{i}}
$$

lo que lleva a:

$$
\tau^{D I D}=[E[Y \mid G=1, T=1]-E[Y \mid G=1, T=0]]
$$

donde $\tau^{D I D}$ es el efecto del tratamiento promedio sobre los tratados medido con la metodología DID. Este modelo puede extenderse fácilmente para incluir un conjunto de covariables $X$, ya que es una función lineal.

Aunque este es un método válido de identificación, los problemas habituales que pueden resultar en estimadores inconsistentes incluyen: dependencia de la forma funcional i.e. los resultados pueden ser diferentes dependiendo de si se usa un modelo en niveles o logaritmos;

7 Véase Angrist and Pischke (2008), Lechner (2010), and Imbens and Wooldridge (2009), entre otros, para una discusión más en detalle de este tipo de estimación. 
heterogeneidad en el efecto del tratamiento; y la imposibilidad de estimar el efecto para el grupo que no fue tratado.

Una alternativa más general al modelo DID es la metodología CIC propuesta por Athey e Imbens $(2002,2006)$. El modelo base para variables continuas se basa en cuatro supuestos:

1. El resultado con tratamiento depende del periodo $T$ y un componente individual no observado $U$. Formalmente:

$$
Y(1)=h(U, T)
$$

2. La distribución de $U$ no varía en el tiempo dentro de un grupo, pero puede variar entre grupos,

$$
U \perp T \mid G
$$

3. $h(u, t)$ es monótona en $u$

4. el soporte de $U \mid G=1$ es un subconjunto del soporte de $U \mid G=0$

En la aproximación CIC la distribución de no observables del grupo de tratamiento puede ser diferente al del grupo de control en formas arbitrarias, pero se asume que no cambia en el tiempo al interior del grupo. Así, el efecto de tratamiento en un momento dado es el mismo para todos los individuos con el mismo conjunto de no observables $U_{i}=u$, indiferentemente de su grupo. El supuesto de monotonicidad estricta no es restrictivo en el caso de variables continuas, pero si lo sería en el caso de que hubiera puntos masivos en la distribución de $Y(0)_{g t}$. Bajo estos supuestos Athey e Imbens demuestran que es posible identificar la distribución de $Y(0) \mid G=1, T=1$; es decir, la distribución ex-post del grupo de tratamiento sin tratamiento. En particular ellos muestran que,

$$
F_{Y(0), 11}(Y)=F_{Y, 10}\left(F_{Y, 00}^{-1}\left(F_{Y, 01}(Y)\right)\right.
$$

donde $F_{Y, g t}(y)$ denota la función de distribución de $Y_{i}$ dado $G_{i}=g, T_{i}=t \quad$, y $F_{Y(0), 11}(y)$

representa la distribución contrafactual del grupo de tratamiento $T=1$ en la ausencia del tratamiento. El valor esperado del contrafactual del grupo de tratamiento sin tratamiento en el segundo periodo esta dado por:

$$
E\left[Y(0) \mid G_{1}=1, T_{1}=1\right]=E\left[F_{Y, 10}\left(F_{Y, 00}^{-1}\left(F_{Y, 01}(Y)\right)\right]\right.
$$

Entonces, el efecto promedio de tratamiento ${ }^{C I C}$ puede escribirse como:

$$
\begin{gathered}
\tau^{C I C}=E\left[Y(1)_{11}-Y(0)_{11}\right]=E\left[Y(1)_{11}\right]-E\left[k^{C I C}\left(Y_{10}\right)\right] \\
=E\left(Y(1)_{11}\right)-E\left[F_{Y, 10}\left(F_{Y, 00}^{-1}\left(F_{Y, 01}(Y)\right)\right]\right.
\end{gathered}
$$


La figura 2 nos muestra de forma más intuitiva en qué consiste la metodología CIC. Tomemos a una persona en el periodo ex-post con un resultado $y$ el cual se ubica en el cuantil $q$. Para construir la distribución del grupo de tratamiento en el segundo periodo en ausencia del tratamiento se siguen los siguientes pasos: primero buscamos a alguien con el mismo valor del resultado $y$ en el grupo de control en el periodo ex-ante y encontramos su cuantil $q$ '. Luego buscamos en la distribución ex-post del grupo de control el valor del resultado $y^{\prime}$ en el cuantil $q^{\prime}$, lo que nos da $\Delta^{C I C}$. Entonces, junto al cuantil original $q$ el valor contrafactual de la distribución es $y+\Delta C I C$. Podemos repetir este procedimiento para cada punto del soporte del grupo de tratamiento; por lo tanto, la distribución contrafactual del resultado estaría dado por $Y_{11}^{N}=F_{01}^{-1}\left(F_{00}\left(Y_{10}\right)\right)$.

Si el supuesto del soporte no se satisface, entonces la distribución contrafactual estaría aun identificada dentro del soporte de $Y_{01}$. Incluso si este no fuera el caso, Athey e Imbens muestran que es posible calcular el efecto del tratamiento en el cuantil $q$ de la distribución de $F_{Y, 10}$ de acuerdo a:

$$
\tau_{q}^{C I C}=F_{Y(1)_{11}}^{-1}(q)-F_{Y(0)_{11}}^{-1}(q)=F_{Y(1)_{11}}^{-1}(q)-F_{Y, 01}^{-1}\left(F_{Y, 00}\left(F_{Y, 10}^{-1}(q)\right)\right)
$$

Este resultado es muy útil si consideramos que el programa puede haber tenido un impacto en la parte baja de la distribución de ingreso, por ejemplo.

El modelo CIC tiene otras extensiones y puede aplicarse también a variables discretas (binarias) como la participación en el mercado de trabajo y obtenerse intervalos del efecto tratamiento promedio. En este caso, se asume que $h(u, t)$ es monótona débil en $u$ y bajo el supuesto de independencia condicional $U \perp G \mid Y, T$ es posible obtener una estimación puntual. El supuesto de independencia condicional en el caso de variables discretas es necesario para asegurar que el nivel de resultados puede compararse entre grupos y de que el cuantil de los resultados sea comparable en el tiempo. También es posible incluir covariables bajo esta metodología y los detalles se encuentran en los apéndices.

\subsubsection{Estimación}

El modelo CIC propuesto por Athey e Imbens considera los casos de variables continuas y discretas. En esta subsección solo consideramos el caso de variables continuas y dejamos el tratamiento de las variables discretas para los apéndices.

Se requieren los supuestos (1)-(4) para asegurar que se cumpla.

$$
\tau^{C I C}=E\left[Y(1)_{11}\right]-E\left[Y(0)_{11}\right]=E\left[Y(1)_{11}\right]-E\left[F_{Y, 01}^{-1}\left(F_{Y, 00}\left(Y_{10}\right)\right)\right]
$$

Adicionalmente, hacemos los siguientes supuestos sobre el proceso generador de datos: 
1. $Y_{i}$ es una selección aleatoria de los sub-población con $G_{i}=g$ durante el periodo $t$, condicionado a $T_{i}=t$ y $G_{i}=g$.

2. Para todo $t, g \in[0,1], \alpha_{g t}=P\left(T_{i}=t, G_{i}=g\right)>0$

3. $Y_{g t}$ son continuas con densidades $f_{Y, g t}(y)$ que son continuamente diferenciables con soporte $Y_{g t}=\left[y_{\min , g t} y_{\max , g t}\right]$

4. El soporte del grupo de tratamiento en el periodo ex-ante es un subconjunto del soporte del grupo de control en el periodo ex-ante.

Así, usamos la distribución empírica como estimador de la función de distribución:

$$
\hat{F}_{Y, g t}(y)=\frac{\sum_{i=1}^{N_{g t}} I\left\{Y_{g t, i} \leq y\right\}}{N_{g t}}
$$

y el estimador de la función inversa de distribución que usamos es:

$$
\hat{F}_{Y, g t}^{-1}(q)=\inf \left\{y \in \mathrm{Y}_{g t}: \hat{F}_{Y, g t}(y) \geq q\right\}
$$

Como es posible que la condición de soporte no se cumpla, entonces estimamos $\hat{F}_{Y(0), 11}(y)$ de acuerdo a:

$$
\hat{F}_{Y(0), 11}(y)\left\{\begin{array}{cc}
0 & \text { si } y<y_{\text {min }, 01} \\
\hat{F}_{Y, 10}\left(\hat{F}_{Y, 00}^{-1}\left(\hat{F}_{Y, 01}(Y)\right)\right) & \text { si } y_{\text {min }, 01} \leq y<y_{\text {max }, 01} \\
1 & \text { en otro caso }
\end{array}\right.
$$

Por lo tanto, (iError! No se encuentra el origen de la referencia.) puede estimarse (consistentemente) a través de:

$$
\hat{\tau}^{C I C}=\frac{\sum_{i=1}^{N_{11}} Y_{11, i}}{N_{11}}-\frac{\sum_{i=1}^{N_{10}} \hat{F}_{01}^{-1}\left(\hat{F}_{00}\left(Y_{10, i}\right)\right)}{N_{10}}
$$

\subsubsection{Inferencia}

Para estimar la varianza del estimador de CIC definimos:

$$
P_{(Y, Z)}=\frac{1}{f_{Y, 01}\left(F_{Y, 01}^{-1}\left(F_{Y, 00}(z)\right)\right)} *\left(I(y \leq z)-F_{Y, 00}(Z)\right)
$$




$$
\begin{gathered}
p(y)=E\left[P\left(y, Y_{10}\right)\right] \\
Q(y, z)=\frac{1}{f_{Y, 01}\left(F_{Y, 01}^{-1}\left(F_{Y, 00}(z)\right)\right)} *\left(I\left\{F_{Y, 01}(y) \leq F_{Y, 00}(z)\right\}-F_{Y, 00}(z)\right) \\
q(y)=E\left[Q\left(y, Y_{10}\right)\right] \\
r(y)=F_{Y, 01}^{-1}\left(F_{Y, 00}(y)\right)-E\left[F_{Y, 01}^{-1}\left(F_{Y, 00}\left(Y_{10}\right)\right)\right] \\
s(y)=y-Y_{11}
\end{gathered}
$$

con varianzas $V^{p}=E\left[p\left(Y_{00}\right)^{2}\right], V^{q}=E\left[q\left(Y_{01}\right)^{2}\right], V^{r}=E\left[r\left(Y_{10}\right)^{2}\right], V^{s}=E\left[s\left(Y_{11}\right)^{2}\right]$

Entonces la distribución asintótica tiene la forma:

$$
\sqrt{N}\left(\hat{\tau}^{C I C}-\tau^{C I C}\right) \rightarrow N\left(\frac{V^{p}}{\alpha_{00}}+\frac{V^{q}}{\alpha_{01}}+\frac{V^{r}}{\alpha_{10}}+\frac{V^{s}}{\alpha_{11}}\right)
$$

La varianza asintótica es estimada reemplazando las expectativas con el promedio de las muestras y usando la función de distribución empírica (y su inversa) mostradas en 11 (y 12). Las funciones de densidad son estimadas usando un Epanechnikov kernel $^{8}$ de manera que

$$
\hat{f}_{Y, 01}(y)=\frac{1}{h N_{01}} \sum_{i=1}^{N_{01}} K\left(\frac{Y_{01, i}-y}{h}\right)
$$

donde el ancho de banda $h$ es especificado de acuerdo a: $h=\frac{1.06 * s d_{Y, 01}}{\sqrt[5]{N_{01}}}$ y $s d$ es la desviación estándar de la muestra de $Y_{01}$

Así, la varianza asintótica de $\sqrt{N}\left(\hat{\tau}^{C I C}-\tau^{C I C}\right)$ es estimada como:

donde $\hat{\alpha}_{g t}=N_{g t} / N$

En la práctica, obtenemos los errores estándar de los estimados a través de bootstrapping. Usamos 1,000 réplicas y calculamos la diferencia entre los cuantiles 0.975 y 0.025 de las

$$
{ }^{8} K(z)\left\{\begin{array}{cc}
\frac{3\left(1-\frac{1}{5} z^{2}\right)}{4 \sqrt{5}} & \text { si }|z|<\sqrt{5} \\
0 & \text { en otro caso }
\end{array}\right\}
$$


muestras del bootstrap y la dividimos por $2 \times 1.96$, de manera que todos los errores estándar sean comparables.

\subsection{Grupos de tratamiento y control}

Definimos los años 2008, 2009 y 2011 como el periodo de tratamiento (T1) y los años 2005, 2006 y 2007 como el periodo de control (T0). Definimos el grupo de tratamiento (G1) como aquellos cuya edad pertenece al intervalo [60,65). Después de todo, esta población fue excluida de las pensiones no contributivas bajo el BONOSOL y fue incluida bajo el esquema de la Renta Dignidad. Sin embargo, hay dos formas alternativas para definir el grupo de control (G0). Por una parte, podemos comparar la población en el intervalo $[60,65)$ con aquellos que son excluidos de la Renta Dignidad en ambos periodos, por ejemplo, las personas cuya edad se encuentra en el intervalo [55,60). En este caso estaríamos obteniendo el impacto de tratar al cohorte de tratamiento. Por otra parte, podríamos comparar la población en el intervalo $[60,65)$ con aquellos que están incluidos en la Renta Dignidad en ambos periodos, por ejemplo, aquellos cuya edad pertenece al intervalo [65,70). En este caso, estaríamos obteniendo el efecto de dejar de tratar el cohorte de tratamiento. Desafortunadamente, para el experimento, el cohorte $[65,70)$ se benefició de un incremento en el monto de la transferencia, de manera que no puede usarse como grupo de control. En el caso de los hogares, utilizamos la máxima edad de los miembros del hogar para determinar la membresía al grupo de control y tratamiento.

Para estudiar la dimensión de género de los efectos en el caso de resultados individuales separamos la muestra de individuos de acuerdo al sexo. En el caso de indicadores de los hogares, hay más opciones para estudiar la dimensión de género. Por ejemplo, podríamos agrupar los hogares de acuerdo al sexo de la cabeza del hogar. Sin embargo, podría darse que el cónyuge esté en el mismo cohorte y por lo tanto no estaríamos comparando mujeres con mujeres y hombres con hombres. En cambio, decidimos agrupar los hogares de acuerdo a la máxima edad en el hogar para definir su estado de tratamiento y luego clasificamos los hogares de acuerdo al sexo de los miembros del hogar en el intervalo de edad relevante. Finalmente, conservamos solo las observaciones con valores para cada una de las características que usamos como controles y usamos factores de expansión en la estimación. Las tablas 3 y 4 muestran los tamaños de muestra finales para hogar e individuos respectivamente.

\subsection{Futuros beneficiarios}

Los futuros beneficiarios pueden estar sujetos a algunos de los incentivos que los beneficiarios directos enfrentan. Por ejemplo, si no es solamente el ingreso no laboral presente sino también futuro que afecta la oferta laboral efectiva de los individuos, entonces la introducción de pensiones no contributivas podría afectar el consumo presente de ocio de los futuros beneficiarios. Para investigar el impacto de la Renta Dignidad en estas personas, comparamos los resultados del cohorte de persones cuya edad todavía no les vuelve beneficiarios, pero que pronto se convertirán después de la reforma de 2007. Así, comparamos las personas en el intervalo $[55,60)$ de edad, que pronto serán beneficiarios, con aquellos que están relativamente lejos de volverse beneficiarios del programa, i.e. el cohorte de personas cuya edad está en el intervalo [50,55). Para diferenciar el impacto del flujo futuro de beneficios de aquél que surge por el beneficio indirecto de vivir con un beneficiario 
directo, restringimos la muestra solamente a aquellos que no están viviendo con un beneficiario (véase la tabla 5).9

\subsubsection{Beneficiarios indirectos}

Los beneficiarios indirectos del programa son personas que no recibieron la pensión no contributiva, pero que estaban residiendo en un hogar con un adulto mayor que era beneficiario del programa y cuya edad está en el intervalo $[60,64)$. Para diferenciar las externalidades de vivir con un adulto mayor de las externalidades de ser un futuro beneficiario restringimos nuestro análisis a la muestra de personas que estaban lejos de volverse beneficiarios, i.e. aquéllos en el intervalo $[25,45)$ de edad. El grupo de control en este caso está conformado por la población en el intervalo $[25,45)$ de edad y que reside con adultos en el intervalo $[55,60$ ) de edad. (véase la tabla 6). Aunque es posible que la pensión no contributiva tenga beneficios indirectos en otros cohortes de edad más jóvenes, el tramo de edad que escogemos es relativamente estable en términos de que las personas participan activamente en el mercado laboral.

\section{Efectos intencionados y no intencionados de la Renta Dignidad}

\subsection{Ingreso, consumo e inversiones del hogar}

\subsubsection{Ingreso per-cápita del hogar}

La tabla 7 muestra el efecto promedio de la Renta Dignidad bajo la metodología DID sobre el ingreso per-cápita del hogar, el ingreso laboral, el ingreso no laboral y las transferencias privadas hacia el hogar per-cápita. En todos los paneles la primera columna muestra el resultado promedio para el cohorte de control [55,60); la segunda columna presenta el resultado promedio para el cohorte de tratamiento $[60,65)$. En ambas columnas, la primera fila muestra el resultado promedio ex-ante mientras que la segunda fila muestra el resultado promedio ex-post. Finalmente, la tercera columna presenta el efecto promedio del tratamiento bajo la metodología DID, o sea, la diferencia ex-post-ex-ante de la diferencia del tratamientocontrol. Los errores estándar están entre corchetes.

Los resultados muestran que la Renta Dignidad incrementa el ingreso del hogar per-cápita un $17 \%$ al $95 \%$ de confianza. Este efecto es causado claramente por un incremento del ingreso no laboral per-cápita, pero también se observa una caída en el ingreso laboral per-cápita que no es significativo. No observamos cambios en las transferencias entre hogares.

Si bien en el caso donde los beneficiarios son hombres no se observan efectos significativos sobre el ingreso per-cápita, en el caso de los hogares donde solamente hay mujeres

9 En el experimento original 164 controles en el periodo ex-ante vivían con un beneficiario, mientras que 582 individuos en el grupo de tratamiento vivían con un beneficiario. 
beneficiarias la Renta Dignidad incrementa significativamente un 36\% el ingreso per-cápita de sus hogares. En el caso donde hay ambos hombres y mujeres beneficiarios en el hogar, no hay un efecto significativo sobre el ingreso per-cápita pero si se observa una reducción significativa en el ingreso laboral per-cápita, lo que nos indica que los efectos positivos de la Renta Dignidad son contrarrestados por las caídas en el ingreso laboral.

A pesar de la importancia de estos resultados, el promedio no nos sirve para estudiar los efectos que podrían producirse en los sectores más pobres o en los más ricos. La figura 3 muestra las funciones de distribución acumulada empíricas del ingreso per-cápita para los grupos de control en la primera fila y los grupos de tratamiento en la segunda. Podemos observar que a diferencia de lo que sucede en los grupos de control, existen diferencias en la parte baja de las distribuciones de los grupos de tratamiento entre los periodos ex-ante y expost.

Las tablas $8,9,10$ y 11 presentan el efecto tratamiento promedio y en los cuantiles de la metodología CIC sobre el ingreso per-cápita del hogar, el ingreso laboral per-cápita, el ingreso no laboral per-cápita y las transferencias entre hogares per-cápita, respectivamente. Los cuantiles se definen en base a la distribución del resultado ex-ante del grupo de tratamiento. Consideramos tanto los estimados condicionados como no condicionados en covariables.

Como se esperaba, existe un incremento significativo en el ingreso no laboral per-cápita entre 246 y $258 \%$ en la muestra de Bolivia. Como muestran los impactos por cuantiles, el efecto de la transferencia se hace más pequeño cuando los hogares tienen ingresos no laborales más grandes. En el caso de las mujeres los efectos sobre el ingreso no laboral son más grandes que en relación a los hombres y en comparación cuando hay hombres y mujeres adultas mayores en el hogar. Este impacto diferencial entre hombres y mujeres se explica porque los hombres tienen un acceso relativamente mayor al sistema de pensiones contributivas y por lo tanto reciben un $75 \%$ de la transferencia solamente.

En el caso del ingreso laboral per-cápita hubieron reducciones significativas a nivel Bolivia entre $30.9 \%$ y $33.3 \%$, lo que en realidad es un resultado inesperado de la Renta Dignidad. Ello implica que el efecto positivo de la Renta Dignidad sobre el ingreso del hogar es contrarrestado en parte por efectos adversos sobre el mercado laboral, los cuales estudiamos más adelante. Un resultado interesante es que se producen reducciones significativas en el ingreso laboral per-cápita de hogares con hombres y mujeres beneficiarios. Es posible que al haber dos beneficiarios de la renta de vejez entonces le sea posible al menos a uno de ellos retirarse del mercado laboral y vivir de la renta.

\subsubsection{Consumo total del hogar per-cápita}

La tabla 12 presenta los estimados de DID para el consumo total per-cápita del hogar, el consumo total per-cápita alimentario y no alimentario. Observamos claramente que no hay efecto alguno sobre cualquiera de estos indicadores de consumo. Ello implica que el incremento en el ingreso debido a la Renta Dignidad no promueve el consumo, lo que contradice los resultados previos a partir de la EPAM.

Para corroborar nuestros resultados, la figura 4 nos muestra las estimaciones de las funciones de distribución acumulada empíricas para el consumo total per-cápita expresado en 
logaritmos. Podemos apreciar que a diferencia de lo que sucede en el caso del ingreso, las diferencias en el grupo de tratamiento y control ex-ante y ex-post no son grandes. La tabla 13 muestra los efectos estimados de CIC para el consumo per-cápita que confirma nuestra hallazgo de que la Renta Dignidad no tuvo un efecto significativo sobre el consumo del hogar. El único caso en el que se observa un incremento significativo es en el caso de hogares con beneficiarias mujeres en el cuantil 20.

Las tablas 14 y 15 confirman que no hay efectos ni sobre el consumo alimentario ni sobre el consumo no alimentario per-cápita de los hogares. Lo más llamativo es que incluso en los cuantiles más bajos de estas distribuciones, particularmente en el caso de alimentos, no hay efectos significativos. Por lo tanto, si los hogares no incrementaron su consumo es posible que hayan destinado el dinero extra a alguna inversión o que simplemente lo hayan ahorrado ya que el programa no hace nada más que transferirles recursos monetarios.

\subsubsection{Inversiones en el hogar}

La tabla 16 muestra los estimados de DID sobre los efectos en inversiones en educación, salud, adquisición de bienes durables e inversiones en la vivienda (reparaciones, ampliaciones, etc.). No observamos efectos de la Renta Dignidad en ninguno de estos tipos de inversión excepto en el caso de inversiones en la vivienda para la muestra de Bolivia. En este caso observamos un incremento de $25 \%$ en las inversiones que realiza el hogar en mejoras de su vivienda con un nivel de confianza de $90 \%$.

Las tablas 17, 18, 19 y 20 presenta las estimaciones de CIC de los efectos de la Renta Dignidad sobre inversiones en educación, salud, adquisición de bienes durables e inversiones en la vivienda, respectivamente. Estos estimados confirman que no hay efectos significativos de la transferencia sobre este tipo de inversiones. En el caso de las inversiones en vivienda observamos un incremento significativo de $20.9 \%$ en la muestra para Bolivia, pero que deja de ser significativo cuando controlamos por covariables.

\subsubsection{Ahorros del hogar}

Ante la evidencia de que los hogares no aumentan su consumo ni su inversión debido a la pensión no contributiva, la única opción restante es que los hogares ahorren la transferencia. La tabla 21 muestra los estimados de DID sobre el nivel de ahorro en Bs. y tres tasas de ahorros diferentes: el ahorro como proporción del ingreso, como proporción del consumo y el ahorro como la diferencia entre los logaritmos del ingreso y del consumo. Los resultados muestran que el ahorro de los hogares beneficiarios se incrementó significativamente. Por ejemplo, en el caso del ahorro como proporción del ingreso el incremento fue de 110 puntos porcentuales y en el caso del ahorro como proporción del consumo el incremento es de 13 puntos porcentuales.

Las muestras de hogares con hombres y hogares con mujeres muestran resultados mixtos en función a la tasa de ahorro analizada. Por ejemplo, en el caso de hogares con beneficiarios hombres no hay un incremento significativo en el ahorro como proporción del ingreso pero si lo hay en el caso del ahorro como proporción del consumo (24 puntos porcentuales). En el caso de las mujeres existe un incremento significativo en el caso del ahorro como proporción del ingreso (169 puntos porcentuales) pero no en el caso del ahorro como proporción del consumo. La tasa de ahorro aproximada a partir de la diferencia entre los logaritmos del 
ingreso y del consumo si muestran incrementos significativos en el caso de hogares con beneficiarios hombres ( 23 puntos porcentuales) y hogares con mujeres beneficiarias (25 puntos porcentuales).

Las tablas 22, 23, 24 y 25 muestran los estimados de CIC sobre el nivel de ahorro, la tasa de ahorro como proporción del ingreso, como proporción del consumo y a partir de la diferencia entre el logaritmo del ingreso y del consumo, respectivamente. Si bien no hay efectos significativos en el nivel de ahorro a nivel de Bolivia, las estimaciones confirman que la tasa de ahorro se incrementó. Más importante aun, los estimados de CIC nos permiten afirmar que tanto los hogares con beneficiarios hombres así como los hogares con beneficiarias mujeres incrementaron su tasa de ahorro significativamente. Por ejemplo, la tasa de ahorro como proporción del ingreso se incremento en 121 puntos porcentuales en la muestra para Bolivia, 214 puntos porcentuales en la muestra de hogares con beneficiarias mujeres solamente y 121 puntos porcentuales en el caso de hogares con beneficiarios hombres solamente. Otro hecho importante es que cuando los hogares tienen un beneficiario hombre y una beneficiaria mujer la tasa de ahorro no se incrementa, lo que se debe a la caída en el ingreso laboral per-cápita de sus hogares. Estos resultados nos permiten afirmar que en promedio los hogares incrementan su ahorro a causa del dinero extra que reciben de la Renta Dignidad.

\subsection{Efectos en indicadores del mercado laboral de beneficiarios directos}

\subsubsection{Nivel y sector de participación}

La tabla 26 muestra los estimadores de DID del efecto promedio de la Renta Dignidad sobre el nivel de participación y sectores de inserción. Nótese que hay diferencias importantes en el nivel y sector de participación entre el cohorte de mayor y el de menor edad. El cohorte más viejo (grupo de tratamiento) no solamente tienen menores niveles de participación en relación al cohorte más joven, sino que también tiene menores niveles de participación en el sector asalariado, ya sea formal o informal. Por otra parte, dadas las diferencias importantes $e x$ post/ex-ante entre los cohortes, los estimadores de DID muestran que la participación en el mercado laboral se ha reducido en 4 puntos porcentuales con un nivel de confianza de $90 \%$ para la muestra de Bolivia. La desagregación por sexo revela que la caída en el nivel de participación puede ser atribuido a la muestra de mujeres, cuya participación cayó en 10 puntos porcentuales. Ahora, si bien la Renta Dignidad afecta negativamente el nivel de participación de las mujeres beneficiarias, no produce efectos en los sectores de participación que sean significativos. La única excepción se produce en el caso de las mujeres en el sector informal asalariado, el cual se reduce en 3 puntos porcentuales al 95\% de confianza.

Las tablas 27 y 28 presentan los estimadores de CIC promedio para el nivel de participación y el sector de participación respectivamente, además de límites superiores e inferiores del efecto. Los resultados confirman que la pensión no contributiva produce una caída significativa en el nivel de participación (entre 6 y 7 puntos porcentuales), el cual es más fuerte en el caso de las mujeres (entre 10 y 12 puntos porcentuales) y no es significativo en el caso de los hombres. La reducción en la participación de las mujeres se debe a reducciones de 2 puntos porcentuales en el sector de trabajo familiar, 3 puntos porcentuales en el sector informal no asalariado, 3 puntos porcentuales en el sector informal asalariado (significativo al $95 \%)$ y 2 puntos porcentuales en el sector formal asalariado. 
Si la reducción se hubiera producido solamente en el sector de empleo familiar (no remunerado) podríamos afirmar con algún grado de confianza que la caída en el nivel de participación se debe a que las mujeres en empleos de mala calidad tienen la posibilidad de retirarse con la pensión no contributiva. No obstante, las caídas se producen en todos los sectores, lo que es señal de un retiro prematuro del mercado de trabajo.

\subsubsection{Intensidad de oferta e ingresos laborales}

Aunque el indicador de participación es interesante por sí mismo, es informativo cuantificar la magnitud del efecto de la pensión no contributiva en la intensidad de oferta y en los salarios e ingresos de los beneficiarios. La tabla 29 muestra los estimadores de DID sobre las horas trabajadas en todos los empleos y en la actividad primaria, en el salario por hora y en los ingresos laborales mensuales, expresadas en logaritmos en cada caso. Encontramos caídas importantes en las horas trabajadas de $18 \%$ en la muestra de Bolivia al $90 \%$ de confianza. En el caso de las mujeres se produce una caída de $40 \%$ en las horas trabajadas que es significativa al 95\%, mientras que en el caso de los hombres no hay efectos significativos. Un gran porcentaje de esta caída se debe principalmente a la reducción de las horas trabajadas en la actividad primaria: $17 \%$ en la muestra de Bolivia y $39 \%$ en la muestra de mujeres.

Estos resultados se confirman a partir de la estimación de CIC para la intensidad de oferta total (tabla 30) y para la actividad primaria ((tabla 31)). Los resultados muestran una caída en la muestra de Bolivia de la intensidad de oferta total entre 22.6 y $24.5 \%$ que son significativas al $90 \%$ de confianza. Nuevamente, la caída más importante en la intensidad de oferta se produce en la muestra de las mujeres (entre 41.8 y 49.5 por ciento al $99 \%$ de confianza) y en el caso de los hombres no hay efectos significativos.

Por otra parte, tanto en la muestra de Bolivia como en la muestra de hombres no se observan efectos significativos en el salario por hora a partir de los estimadores de DID. Sin embargo si se produce una caída significativa de $67 \%$ en el salario por hora en la muestra de mujeres. Combinados con la disminución en la intensidad de oferta, el ingreso laboral mensual de las mujeres cae $75 \%$ a un nivel de confianza de $95 \%$. Estos resultados son corroborados por los estimadores de CIC, que indican una caída entre 68.9 y $71.4 \%$ en el salario por hora de las mujeres y entre 74.2 y $81.2 \%$ en su ingreso laboral mensual.

\subsection{Efectos en indicadores del mercado laboral de futuros beneficiarios}

\subsubsection{Nivel y sector de participación}

Las tablas 34 muestran los estimadores de DID para la participación y sector de empleo de los futuros beneficiarios y los respectivos estimados de CIC se encuentran en las tablas 35 y 36 . Los resultados muestran incrementos de 3 puntos porcentuales pero que son significativos solamente con el enfoque de DID y a un nivel de $90 \%$, por lo que los resultados no son robustos. Hay resultados mixtos en el caso de los sectores de empleo ya que hay efectos negativos en el sector de empleo familiar (4 puntos porcentuales a partir de DID y CIC) y en el sector informal asalariado (1 punto porcentual con DID y CIC), significativos en el primer caso; pero efectos positivos en el sector informal no asalariado (6 puntos porcentuales con 
DID y CIC, significativos al 95\%) y formal asalariado (2 puntos porcentuales con DID y CIC, no significativos).

La desagregación por sexo muestra que la inserción en el sector de trabajo familiar no remunerado de las mujeres se reduce en 6 puntos porcentuales (DID y CIC) y que la inserción de las mujeres en el sector formal asalariado de los futuros beneficiarios aumenta entre 4 puntos porcentuales (DID) y 3 puntos porcentuales (CIC). En el caso de los hombres solo se observa un incremento significativo en la inserción en el sector informal no asalariado de 6 puntos porcentuales (DID y CIC) significativo al $90 \%$.

\subsubsection{Intensidad de oferta e ingresos laborales}

La tabla 37 muestra los estimados de DID para las horas trabajadas en todas las actividades, las horas trabajadas en la actividad primaria, el salario por hora y el ingreso laboral mensual. Los respectivos estimados de CIC se encuentran en las tablas 38, 39, 40 y 41.

En el caso de las horas trabajadas en total no hay efectos significativos en la muestra de Bolivia, sin embargo si hay efectos positivos entre 16\% (DID) y $14.2 \%$ (CIC) en las horas trabajadas al mes en la actividad primaria. Las estimaciones de DID y CIC muestran incrementos significativos en el salario por hora en la muestra de Bolivia de $64 \%$ en el caso de DID y entre 55\% y $65 \%$ en el caso de CIC. A razón de estos incrementos en el salario por hora, el ingreso laboral mensual en la muestra de Bolivia crece $61 \%$ usando DID al $99 \%$ de confianza y entre 52 y $63 \%$ usando estimadores de CIC al $95 \%$ de confianza.

Los estimadores de DID desagregados por sexo muestran que hubieron incrementos significativos en el salario por hora (71\%) y el ingreso laboral mensual (78\%) de las futuras mujeres beneficiarias. Estos efectos son corroborados por los estimadores de CIC, que muestran un incremento entre 64 y $66 \%$ en el salario y $73 \%$ en el ingreso laboral mensual de las mujeres. En el caso de los hombres los estimadores de DID muestran efectos positivos significativos tanto en la intensidad de oferta como en los salarios. Sin embargo estos mismos resultados no son significativos usando estimadores de CIC aun después de controlar por covariables, por lo que los resultados no son robustos.

\subsection{Efectos en indicadores del mercado laboral de beneficiarios indirectos}

La tabla 42 muestra las estimaciones de DID de los efectos sobre el nivel y sector de empleo de los beneficiarios indirectos del programa. Si bien se observan caídas en los niveles de participación de los beneficiarios indirectos en el intervalo $[25,44)$ de edad, los efectos no son significativos. Más aun, no hay cambios significativos en los sectores de participación. La desagregación por sexo tampoco muestra cambios significativos en los niveles de participación y sectores de empleo de hombres y mujeres. La ausencia de efectos de la pensión no contributiva sobre beneficiarios indirectos se confirma con los estimados no significativos de CIC que se observan en las tablas 43 y 44 para la participación y sector de empleo respectivamente.

La tabla 45 muestra las estimadores de DID para las horas trabajadas en total, las horas trabajadas en la actividad primaria, el salario por hora y el ingreso laboral mensual. Las 
tablas 46, 47, 48 y 49 muestran los estimadores respectivos de CIC. Los resultados, tanto con DID y CIC, muestran disminuciones en la intensidad de oferta de los beneficiarios indirectos que sin embargo no son significativas. La desagregación por sexo tampoco muestra resultados significativos e incluso muestra una disminución en la oferta laboral de las mujeres indirectamente beneficiadas. Este resultado es interesante porque si bien el nivel de participación en el mercado laboral de los beneficiarios directos disminuye a causa de la pensión no contributiva, su mayor disponibilidad de tiempo no se traduce en que las mujeres aumenten su oferta laboral e igualen la de los hombres. Visto de otra forma, el retiro prematuro de los beneficiarios no es compensado por una mayor oferta laboral de quienes aún se encuentran en el mercado laboral.

\section{Conclusiones e implicaciones de política}

Este documenta presenta los resultados de una evaluación cuasi-experimental de la Renta Dignidad, una pensión universal no contributiva para los adultos mayores implementada en Bolivia. Para identificar los efectos de recibir la pensión aprovechamos una variación exógena de política que reduce el criterio de edad para ser elegible de 65 a 60 años en diciembre de 2007. Aplicando los enfoques de Diferencias-en-Diferencias (DID) y Cambiosen-Cambios (CIC) a una muestra combinada de tres años de datos de sección cruzada antes y después del cambio exógeno en política estimamos no solamente efectos promedio sino también efectos en cuantiles.

Nuestros hallazgos principales se resumen bien por el título de nuestro documento. Al menos en el caso Boliviano, encontramos que las transferencias no contributivas tienen complejos efectos intencionados y no intencionados sobre diferentes indicadores del hogar e individuales para diferentes tipos de individuos y hogares. En el caso de las mujeres, encontramos que las pensiones no contributivas han, en promedio, incrementado el ingreso no laboral de sus hogares, lo que ha reducido su oferta laboral y sus ingresos laborales, lo que por ende ha reducido el ingreso laboral total de sus hogares. De esta manera, ceteris paribus, el efecto total del programa en el ingreso per-cápita del hogar se reduce.

En el caso de los hombres la transferencia no contributiva incrementó el ingreso no laboral total de sus hogares, en menor porcentaje en relación a las mujeres, pero no hubieron efectos significativos sobre su oferta laboral y el efecto positivo sobre el ingreso per-cápita del hogar no es significativo. Este resultado se explica en parte por un mayor acceso de los hombres al sistema de pensiones contributivas en relación a las mujeres, convirtiéndolas más dependientes de la transferencia.

Nuestros resultados también sugieren que los recursos adicionales no fueron ni consumidos ni invertidos en educación, salud, vivienda o la adquisición de bienes durables. Los recursos adicionales en cambio fueron ahorrados por los hogares, un comportamiento que es consistente con el perfil de ahorro del cohorte estudiado.

El principal mensaje de política que podríamos extraer de estos resultados es que las transferencias no contributivas tienen no solamente efectos intencionados sino también efectos no intencionados sobre los beneficiarios directos y futuros. Por lo tanto, es muy importante calibrar el tamaño de la transferencia para maximizar las consecuencias intencionadas y minimizar las no intencionadas. Incrementar la cantidad de recursos disponibles a las personas puede no traducirse en un incremento uno-a-uno en el ingreso del 
hogar si es que hay efectos ingreso que reducen la oferta laboral de los beneficiarios efectivos; y, en el caso de la población adulta mayor, si les dan el incentivo de retirarse prematuramente del mercado laboral con un ingreso seguro. Ciertamente es importante evaluar las ganancias potenciales asociadas con el incremento en el ocio, nivel de satisfacción vs. el costo monetario asociado no solamente con las pérdidas en producción y costos directos del programa. En el caso particular de Bolivia y dada la transición demográfica hacia una población más adulta, se debe considerar una estrategia de focalización en el futuro cercano.

\section{Referencias}

[1] J. Angrist and J.-S. Pischke, Mostly Harmless Econometrics: An Empiricist's Companion. 2008.

[2] S. Athey and G. Imbens, "Supplementary Materials for: 'Identification and Inference in Nonlinear Difference-in-Differences Models"," 2006.

[3] S. Athey and G. Imbens, "Identification and Inference in Nonlinear Difference-inDifferences Models.” 2002.

[4] S. Athey and G. Imbens, "Identification and Inference in Nonlinear Difference-inDifferences Models," Econometrica, vol. 74, no. 2, pp. 431-497, 2006.

[5] M. Bertrand, E. Duflo, and S. Mullainathan, "How Much Should We Trust Differencesin-Differences Estimates?," vol. 119, no. 1, pp. 249-275, 2004. 
[6] C. Chaisemartin, "Fuzzy Differences in Differences." 2012.

[7] C. Chaisemartin and X. D'Haultfoeuille, "Fuzzy Changes-in-Changes.” 2014.

[8] G. Imbens and J. Wooldridge, "Difference-in-Differences Estimation,” 2007.

[9] G. Imbens and J. Wooldridge, "Recent Developments in the Econometrics of Program Evaluation." 2008.

[10] M. Lechner, "The Estimation of Causal Effects by Difference-in-Difference Methods," Foundations and Trends in Econometrics, vol. 4, no. 3, pp. 165-224, 2010.

[11] O. Ropponen, Applications of treatment effects models and semiparametric estimation. 2011.

[12] S. Martinez, "Pensions, poverty and household investments in Bolivia." 2004.

[13] W. Hernani-Limarino and P. Villarroel, "Effects of Renta Dignidad on labour market outcomes of the elderly." Forthcoming.

[14] W. Hernani-Limarino and M. Claure, "Reassessing the effects of Renta Dignidad using EPAM." 2014.

[15] M. Yanez-Pagans, "Culture and Human Capital Investments : Evidence of an Unconditional Cash Transfer Program in Bolivia," 2008.

[15] W. Hernani-Limarino and A. Eid, "Entrepreneurship and Economic Mobility: A Case of Study of Bolivia." 2012.

[16] F. Escobar, S. Martínez, and J. Mendizábal, "El impacto de la Renta Dignidad: Política de Redistribucion del Ingreso, Consumo y Reduccion de la Pobreza en Hogares con Personas Adultas Mayores.” 2013.

[17] R. Rofman and M. Oliveri, La cobertura de los sistemas previsionales en América Latina: conceptos e indicadores, vol. 7 of Serie de Documentos de Trabajo sobre Politicas Sociales. 2011. 
Figura 1: Evolución de la participación en el sector formal y acceso a pensiones contributivas por sexo

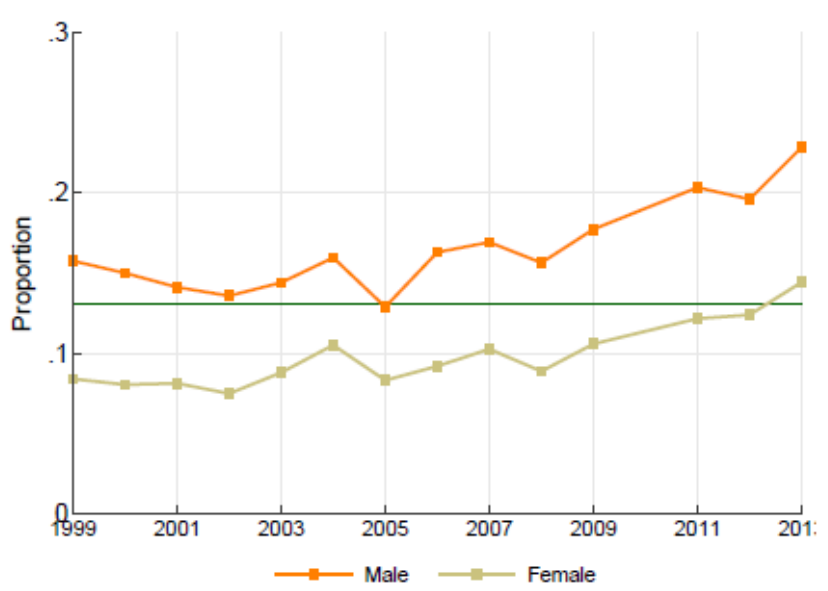

(a) Empleo formal

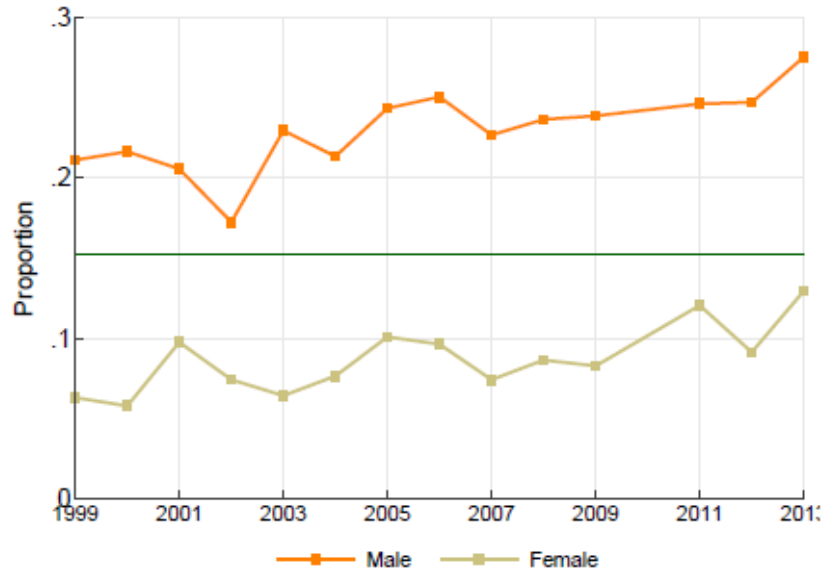

(b) Acceso a pensión contributiva 
Fuente: Estimación de los autores basada en la serie armonizada por Fundación ARU de encuestas de hogares (INE).

Notas: Definimos a las personas entre 25 y 65 años como la población en edad de trabajar. Un trabajador se encuentra en un empleo formal si está afiliado al sistema de seguridad social de largo plazo, e.g., contribuye a AFP. El acceso a pensión contributiva se mide sobre la población mayor a 65 años.

Tabla 1: Esquemas de pensiones no contributivas en Bolivia

\begin{tabular}{|c|c|c|c|c|c|c|}
\hline & $\begin{array}{c}1997 \\
\text { BONOSOL }\end{array}$ & \multicolumn{3}{|c|}{ BOLIVIDA } & $\begin{array}{l}2003-2007 \\
\text { BONOSOL }\end{array}$ & Renta Dignidad \\
\hline Beneficiarios & 65 o más & \multicolumn{3}{|c|}{650 más } & 65 o más & 60 o más \\
\hline $\begin{array}{l}\text { con pensión contributiva } \\
\text { sin pensión contributiva }\end{array}$ & Bs. 1,300 & Suspendido & Bs. $790^{1}$ & Bs. $840^{2}$ & Bs. 1,800 & $\begin{array}{ll}\text { Bs. } 150(1,800) & \text { Bs. } 200(2,400) \\
\text { Bs. } 200(2,400) & \text { Bs. } 250(3,000)\end{array}$ \\
\hline Frecuencia & Anual & \multicolumn{3}{|c|}{ Anual } & Anual & Mensual (anual) \\
\hline
\end{tabular}

\footnotetext{
${ }^{1}$ Dos pagos de Bs. 395 correspondientes a los años 1998 y 1999.

2 Dos pagos de Bs. 420 correspondientes a los años 2000 y 2001.
}

Fuente: Elaboración de los autores.

Tabla 2: Fuentes de información disponible para estudiar la Renta Dignidad 


\begin{tabular}{|c|c|c|c|c|}
\hline & EH '05-'11 & EH 2011 & EPAM 2011 & ETE '09-'10 \\
\hline Muestra de hogares & 29,157 & 8,851 & 2,748 & $? ?$ \\
\hline $\max ($ edad $) \in[55,60)$ & 2,483 & 780 & 670 & $? ?$ \\
\hline $\max ($ edad $) \in[60,65)$ & 2,108 & 666 & 620 & $? ?$ \\
\hline $\max ($ edad $) \in[65,70)$ & 1,865 & 572 & 547 & $? ?$ \\
\hline \multicolumn{5}{|c|}{ Indicadores de bienestar del hogar } \\
\hline Ingreso & $\mathrm{X}$ & $\mathrm{X}$ & $\mathrm{X}$ & $\mathrm{X}$ \\
\hline Consumo & $\mathrm{X}$ & $\mathrm{X}$ & $\mathrm{X}$ & n.a. \\
\hline \multicolumn{5}{|c|}{ Indicadores de ahorro e inversión } \\
\hline Ahorro & $\mathrm{X}$ & $\mathrm{X}$ & $\mathrm{X}$ & n.a. \\
\hline Gasto en educación & $\mathrm{X}$ & $\mathrm{X}$ & $\mathrm{X}$ & n.a. \\
\hline Gasto en salud & $\mathrm{X}$ & $\mathrm{X}$ & $?$ & n.a. \\
\hline Gasto en durables & $\mathrm{X}$ & $\mathrm{X}$ & n.a. & n.a. \\
\hline Inversiones en la vivienda & $\mathrm{X}$ & $\mathrm{X}$ & n.a. & n.a. \\
\hline Muestra total de individuos & 114,726 & 33,821 & 9,158 & $? ?$ \\
\hline edad $\in[55,60)$ & 3,889 & 1,242 & 1,109 & $? ?$ \\
\hline edad $\in[60,65)$ & 3,132 & 982 & 925 & $? ?$ \\
\hline edad $\in[65,70)$ & 2,581 & 779 & 772 & ?? \\
\hline \multicolumn{5}{|l|}{ Oferta en el mercado laboral } \\
\hline Participación & $\mathrm{X}$ & $\mathrm{X}$ & $\mathrm{X}$ & $\mathrm{X}$ \\
\hline Horas trabajadas & $\mathrm{X}$ & $\mathrm{X}$ & n.a. & $\mathrm{X}$ \\
\hline \multicolumn{5}{|l|}{ Sector de empleo } \\
\hline Trabajo familiar & $\mathrm{X}$ & $\mathrm{X}$ & $\mathrm{X}$ & $\mathrm{X}$ \\
\hline Cuenta propia & $\mathrm{X}$ & $\mathrm{X}$ & $\mathrm{X}$ & $\mathrm{X}$ \\
\hline Informal asalariado & $\mathrm{X}$ & $\mathrm{X}$ & $?$ & $\mathrm{X}$ \\
\hline Formal asalariado & $\mathrm{X}$ & $\mathrm{X}$ & n.a. & $\mathrm{X}$ \\
\hline
\end{tabular}

Fuente: Elaboración de los autores.

Notas: "X"= disponible, "?"= incompleto, "n.a."= no disponible, "??"= desconocido. EH=Encuesta de Hogares; EPAM=Encuesta a hogares con Personas Adultas Mayores; ETE= Encuesta Trimestral de Empleo.

Figura 2: Transformaciones 


\section{Group 0 Distributions}

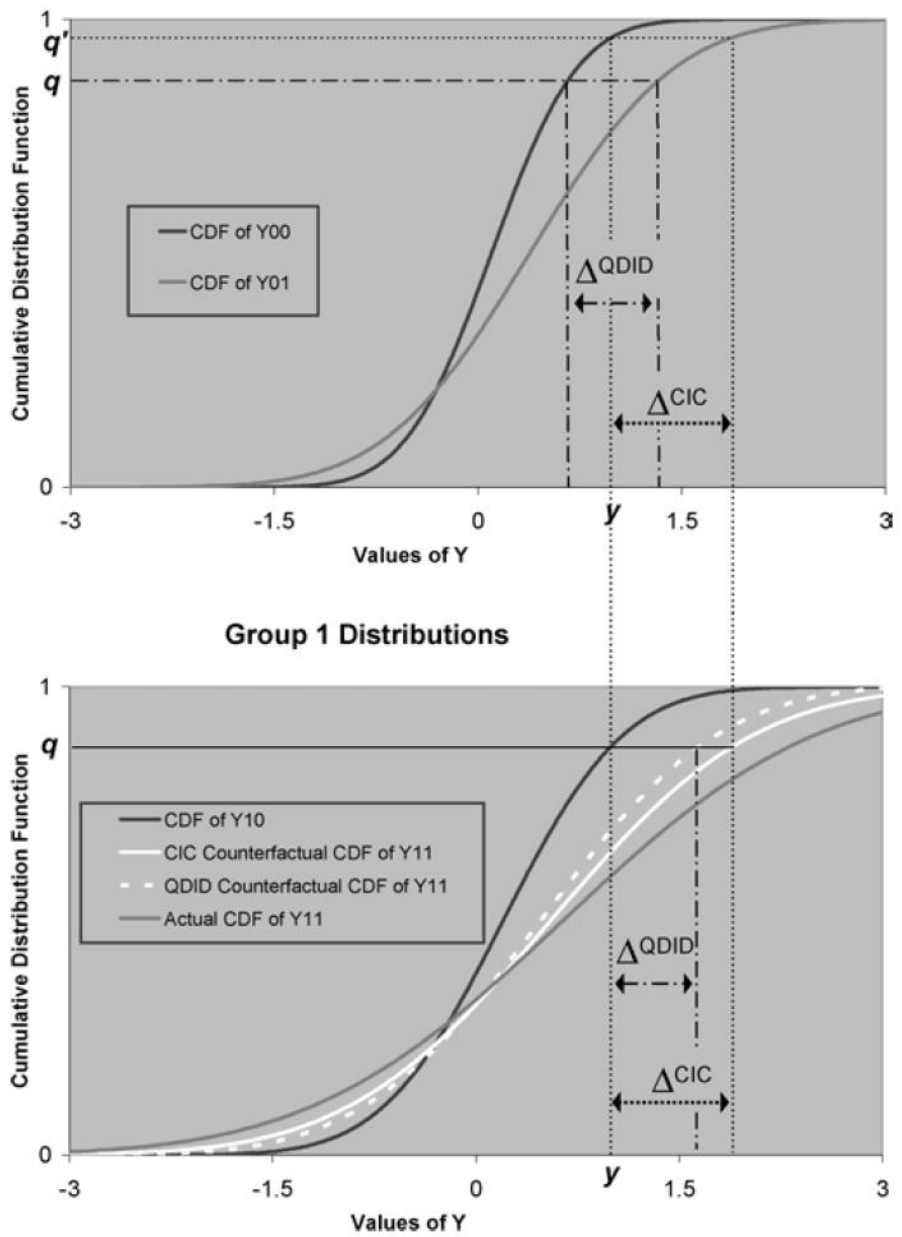

Fuente: Extraído de Athey e Imbens (2006).

Tabla 3: Tamaño de muestra: hogares 


\begin{tabular}{|c|c|c|c|c|}
\hline & \multicolumn{2}{|c|}{$\mathrm{G} 0=\operatorname{edad}_{\text {hhmaxage }} \in[55,60)$} & \multicolumn{2}{|c|}{ G1 $=$ edad $_{\text {hhmaxage }} \in[60,65)$} \\
\hline \multirow{4}{*}{$\mathrm{T} 0=2005-2007$} & Bolivia & $=953$ & Bolivia & $=791$ \\
\hline & solo hombres G0 & $=434$ & solo hombre adulto mayor & $=370$ \\
\hline & solo mujeres G0 & $=336$ & solo mujer adulto mayor & $=286$ \\
\hline & hombre y mujer & $=183$ & hombre y mujer & $=135$ \\
\hline & Bolivia & $=1,491$ & Bolivia & $=1,289$ \\
\hline & only G0 men & $=709$ & solo hombre adulto mayor & $=603$ \\
\hline $11=2008-2011$ & only G0 women & $=509$ & solo mujer adulto mayor & $=460$ \\
\hline & hombre y mujer & $=273$ & hombre y mujer & $=226$ \\
\hline
\end{tabular}

Fuente: Estimación de los autores basada en la serie armonizada por Fundación ARU de encuestas de hogares (INE).

Nota: Solo se conservan las observaciones que no tengan valores cero o perdidos en el ingreso, consumo y ahorro. Dejamos de lado las observaciones que no tengan valores observados en todos los controles usados.

Tabla 4: Tamaño de muestra: individuos

\begin{tabular}{|c|c|c|c|c|}
\hline & $\mathrm{G} 0=\mathrm{eda}$ & $i_{i} \in[55,60)$ & $\mathrm{G} 1=\mathrm{edac}$ & ${ }_{i} \in[60,65)$ \\
\hline \multirow{3}{*}{$\mathrm{T} 0=2005-2007$} & Bolivia & $=1,519$ & Bolivia & $=1,217$ \\
\hline & hombre & $=716$ & hombre & $=581$ \\
\hline & mujer & $=803$ & mujer & $=636$ \\
\hline \multirow{3}{*}{$\mathrm{T} 1=2008-2011$} & Bolivia & $=2,364$ & Bolivia & $=1,912$ \\
\hline & hombre & $=1,157$ & hombre & $=944$ \\
\hline & mujer & $=1,207$ & mujer & $=968$ \\
\hline
\end{tabular}

Fuente: Estimación de los autores basada en la serie armonizada por Fundación ARU de encuestas de hogares (INE).

Nota: Dejamos de lado las observaciones que no tengan valores observados en todos los controles usados.

Tabla 5: Tamaño de muestra: futuros beneficiarios 


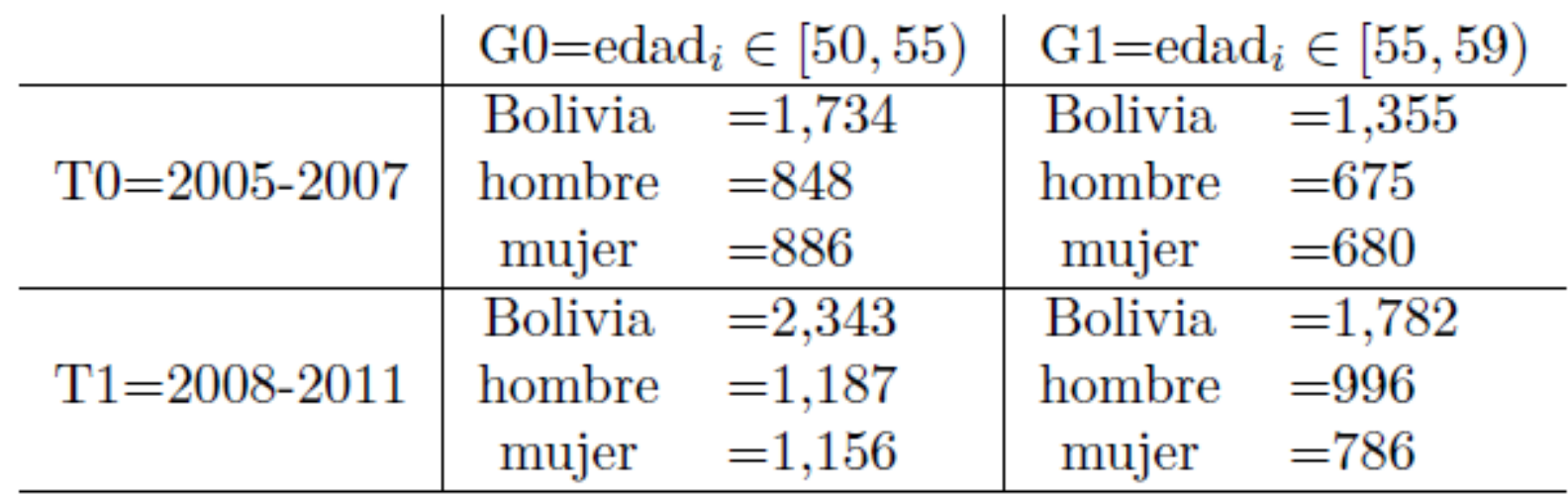

Fuente: Estimación de los autores basada en la serie armonizada por Fundación ARU de encuestas de hogares (INE).

Nota: Dejamos de lado las observaciones que no tengan valores observados en todos los controles usados.

Tabla 6: Tamaño de muestra: beneficiarios indirectos

\begin{tabular}{c|c|cc} 
& $\begin{array}{c}\text { G0=edad } \\
\text { edad }\end{array}$ hhmaxedad $\in[25,45) \mathrm{y}$ & G1=edad & $\in[25,45) \mathrm{y}$ \\
& Bolivia $=577$ & Bolivia $=542$ \\
T0=2005-2007 & hombre $=283$ & hombre $=258$ \\
& mujer $=294$ & mujer $=284$ \\
\hline \multirow{3}{*}{$\mathrm{T} 1=2008-2011$} & Bolivia $=895$ & Bolivia $=777$ \\
& hombre $=470$ & hombre $=374$ \\
& mujer $=425$ & mujer $=403$ \\
\hline
\end{tabular}

Fuente: Estimación de los autores basada en la serie armonizada por Fundación ARU de encuestas de hogares (INE).

Nota: Dejamos de lado las observaciones que no tengan valores observados en todos los controles usados.

Tabla 7: Estimados de Diff-in-Diff de indicadores de ingreso del hogar 


\begin{tabular}{|c|c|c|c|c|c|c|c|c|c|c|c|c|}
\hline & \multicolumn{3}{|c|}{ Bolivia } & \multicolumn{3}{|c|}{ Solo hombres IAI } & \multicolumn{3}{|c|}{ Solo mujeres IAI } & \multicolumn{3}{|c|}{ Mujer y hombre IAI } \\
\hline & {$[55,60)$} & {$[60,65)$} & DID & {$[55,60)$} & {$[60,65)$} & DID & {$[55,60)$} & {$[60,65)$} & DID & {$[55,60)$} & {$[60,65)$} & DID \\
\hline \multicolumn{13}{|c|}{ ingreso per-cápita del hogar (log Bs. 2012 al mes) } \\
\hline \multirow[t]{2}{*}{$\mathrm{T}=0$} & 6.32 & 6.19 & & 6.39 & 6.29 & & 6.32 & 6.09 & & 6.18 & 6.12 & \\
\hline & {$[0.05]$} & {$[0.05]$} & 0.17 & {$[0.07]$} & {$[0.08]$} & 0.14 & {$[0.07]$} & {$[0.09]$} & 0.36 & {$[0.11]$} & {$[0.13]$} & -0.11 \\
\hline \multirow[t]{2}{*}{$\mathrm{T}=1$} & 6.51 & 6.55 & {$[0.09]^{* *}$} & 6.57 & 6.61 & {$[0.13]$} & 6.46 & 6.60 & {$[0.14]^{* * *}$} & 6.45 & 6.28 & {$[0.20]$} \\
\hline & {$[0.03]$} & {$[0.03]$} & & {$[0.05]$} & {$[0.05]$} & & {$[0.06]$} & {$[0.05]$} & & {$[0.08]$} & {$[0.08]$} & \\
\hline \multicolumn{13}{|c|}{ ingreso laboral del hogar per-cápita (log Bs. 2012 al mes) } \\
\hline \multirow[t]{2}{*}{$\mathrm{T}=0$} & 5.75 & 5.29 & & 6.06 & 5.62 & & 5.43 & 4.76 & & 5.62 & 5.51 & \\
\hline & {$[0.07]$} & {$[0.09]$} & -0.22 & {$[0.09]$} & {$[0.11]$} & 0.02 & {$[0.13]$} & {$[0.17]$} & -0.17 & {$[0.16]$} & {$[0.17]$} & -0.96 \\
\hline \multirow[t]{2}{*}{$\mathrm{T}=1$} & 6.18 & 5.50 & [0.14] & 6.30 & 5.88 & [0.19] & 5.97 & 5.14 & {$[0.28]$} & 6.26 & 5.19 & {$[0.31]^{* * *}$} \\
\hline & {$[0.05]$} & {$[0.08]$} & & {$[0.07]$} & {$[0.10]$} & & {$[0.10]$} & {$[0.15]$} & & {$[0.09]$} & {$[0.18]$} & \\
\hline \multicolumn{13}{|c|}{ ingreso no laboral del hogar per-cápita (log Bs. 2012 al mes) } \\
\hline \multirow[t]{2}{*}{$\mathrm{T}=0$} & 1.84 & 2.45 & & 1.16 & 2.16 & & 2.60 & 2.65 & & 2.00 & 2.85 & \\
\hline & {$[0.11]$} & {$[0.13]$} & 2.46 & {$[0.16]$} & {$[0.18]$} & 1.89 & {$[0.19]$} & {$[0.21]$} & 3.04 & {$[0.24]$} & {$[0.28]$} & 2.71 \\
\hline \multirow[t]{2}{*}{$\mathrm{T}=1$} & 1.20 & 4.28 & {$[0.20]^{* * *}$} & 0.98 & 3.86 & {$[0.30]^{* * *}$} & 1.64 & 4.72 & {$[0.34]^{* * *}$} & 0.93 & 4.49 & {$[0.45]^{* * * *}$} \\
\hline & {$[0.09]$} & {$[0.08]$} & & {$[0.12]$} & {$[0.12]$} & & {$[0.15]$} & [0.11] & & {$[0.20]$} & {$[0.17]$} & \\
\hline \multicolumn{13}{|c|}{ ingreso por transferencias privadas hacia el hogar per-cápita (log Bs. 2012 al mes) } \\
\hline \multirow[t]{2}{*}{$\mathrm{T}=0$} & -0.09 & 0.11 & & -0.77 & -0.41 & & 0.72 & 0.68 & & -0.01 & 0.34 & \\
\hline & {$[0.11]$} & {$[0.12]$} & 0.00 & [0.13] & {$[0.16]$} & -0.39 & {$[0.21]$} & {$[0.22]$} & 0.40 & {$[0.23]$} & {$[0.28]$} & 0.17 \\
\hline \multirow[t]{2}{*}{$\mathrm{T}=1$} & -0.39 & -0.18 & {$[0.21]$} & -0.63 & -0.66 & {$[0.27]$} & 0.19 & 0.54 & {$[0.39]$} & -0.88 & -0.37 & {$[0.45]$} \\
\hline & {$[0.09]$} & {$[0.10]$} & & {$[0.12]$} & {$[0.12]$} & & {$[0.16]$} & {$[0.19]$} & & {$[0.18]$} & {$[0.21]$} & \\
\hline
\end{tabular}

Fuente: Estimación de los autores basada en la serie armonizada por Fundación ARU de encuestas de hogares (INE).

Notas: Errores estándar entre corchetes. El error estándar del estimador de DID se estima como $\sqrt{e e_{00}^{2}+e e_{01}^{2}+e e_{10}^{2}+e e_{11}^{2}}$ donde, por ejemplo, $e e_{11}$ es el error estándar del cohorte $[60,65)$ en el periodo $T=1$. IAI=en el intervalo de edad (in age interval). 
Figura 3: Función de distribución acumulada empírica del (log) ingreso per-cápita del hogar

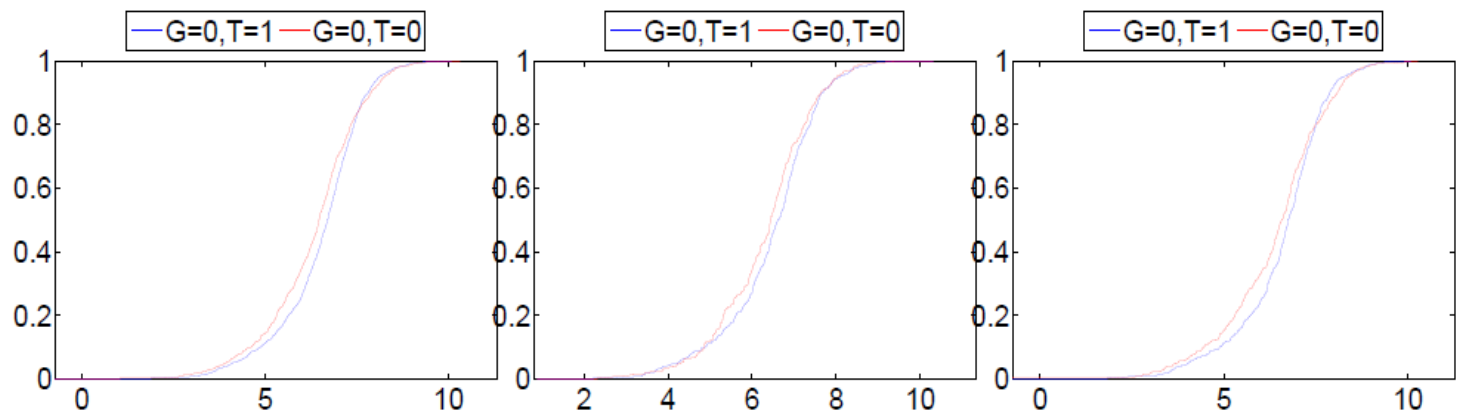

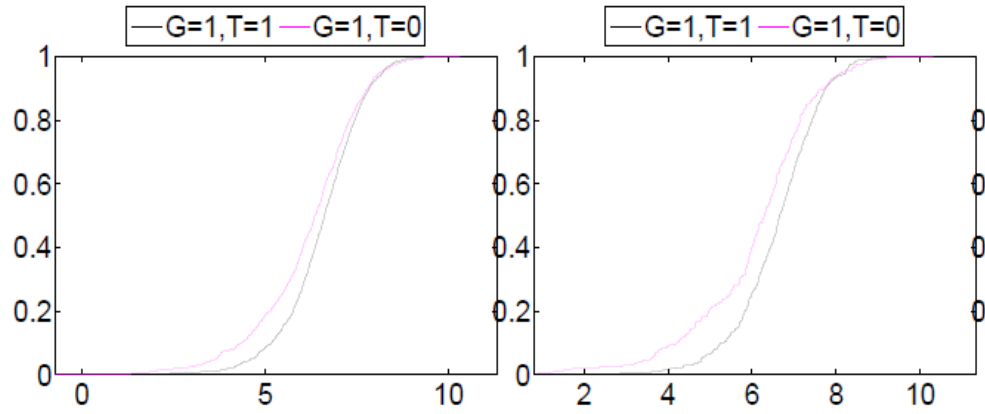

(a) Bolivia

(b) Mujeres

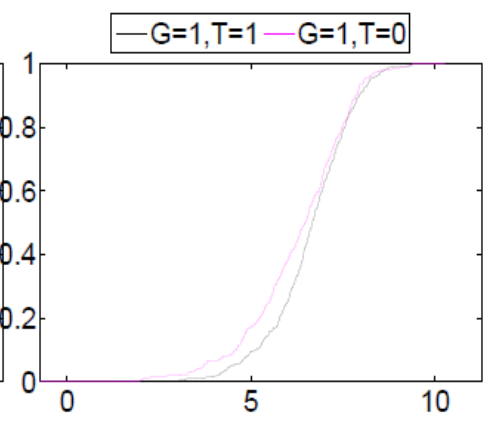

(c) Hombres

Fuente: Estimación de los autores basada en la serie armonizada por Fundación ARU de encuestas de hogares (INE). 
Tabla 8: Efecto sobre el $(\log )$ del ingreso per-cápita del hogar, Changes-in-Changes.

\begin{tabular}{|c|c|c|c|c|c|c|}
\hline & CIC $C_{\text {promedio }}$ & $C I C_{q 0,1}$ & $C I C_{q 0,2}$ & $C I C_{q 0,5}$ & $C I C_{q 0,8}$ & $C I C_{q 0,9}$ \\
\hline \multicolumn{7}{|c|}{ Bolivia } \\
\hline \multirow[t]{2}{*}{$\tau^{C I C}$} & 0.170 & 0.583 & 0.288 & 0.047 & 0.069 & 0.172 \\
\hline & {$[0.081]^{* *}$} & {$[0.229]^{* *}$} & {$[0.172]^{*}$} & {$[0.089]$} & {$[0.082]$} & {$[0.099]^{*}$} \\
\hline \multirow[t]{2}{*}{$\tau_{w / \operatorname{cov}}^{C I C}$} & 0.155 & 0.541 & 0.436 & 0.050 & -0.106 & 0.047 \\
\hline & {$[0.130]$} & {$[0.285]^{*}$} & {$[0.251]^{*}$} & {$[0.153]$} & {$[0.157]$} & {$[0.144]$} \\
\hline \multicolumn{7}{|c|}{ Mujeres } \\
\hline \multirow{2}{*}{$\tau^{C I C}$} & 0.371 & 1.286 & 0.783 & 0.219 & 0.161 & 0.004 \\
\hline & {$[0.138]^{* * *}$} & {$[0.378]^{* * *}$} & {$[0.405]^{*}$} & {$[0.142]$} & {$[0.125]$} & {$[0.212]$} \\
\hline \multirow{2}{*}{$\tau_{w / c o v}^{C I C}$} & 0.476 & 1.392 & 0.970 & 0.298 & 0.037 & 0.171 \\
\hline & {$[0.218]^{* *}$} & {$[0.462]^{* * *}$} & {$[0.433]^{* *}$} & {$[0.242]$} & {$[0.289]$} & [0.259] \\
\hline \multicolumn{7}{|c|}{ Hombres } \\
\hline \multirow[t]{2}{*}{$\tau^{C I C}$} & 0.128 & 0.174 & 0.198 & -0.021 & 0.063 & 0.268 \\
\hline & {$[0.127]$} & {$[0.375]$} & {$[0.268]$} & {$[0.133]$} & {$[0.101]$} & {$[0.136]^{* *}$} \\
\hline \multirow[t]{2}{*}{$\tau_{w / \operatorname{cov}}^{C I C}$} & 0.046 & 0.009 & 0.102 & 0.037 & -0.105 & 0.115 \\
\hline & {$[0.181]$} & {$[0.461]$} & {$[0.347]$} & {$[0.207]$} & {$[0.229]$} & {$[0.210]$} \\
\hline \multicolumn{7}{|c|}{ Mujer y hombre } \\
\hline \multirow[t]{2}{*}{$\tau^{C I C}$} & -0.133 & 0.096 & 0.075 & -0.393 & -0.151 & -0.055 \\
\hline & {$[0.189]$} & {$[0.450]$} & {$[0.337]$} & {$[0.198]^{* *}$} & {$[0.140]$} & {$[0.244]$} \\
\hline \multirow{2}{*}{$\tau_{w / \operatorname{cov}}^{C I C}$} & -0.207 & -0.406 & 0.003 & -0.611 & -0.239 & -0.285 \\
\hline & {$[0.286]$} & {$[0.646]$} & {$[0.430]$} & {$[0.416]$} & {$[0.392]$} & [0.411] \\
\hline
\end{tabular}

Fuente: Estimación de los autores basada en la serie armonizada por Fundación ARU de encuestas de hogares (INE). 
Tabla 9: Efectos sobre el (log) del ingreso laboral per-cápita del hogar, estimador Changes-inChanges

\begin{tabular}{|c|c|c|c|c|c|c|}
\hline & CIC $C_{\text {promedio }}$ & $C I C_{q 0,1}$ & $C I C_{q 0,2}$ & $C I C_{q 0,5}$ & $C I C_{q 0,8}$ & $C I C_{q 0,9}$ \\
\hline Bol & & & & & & \\
\hline$\tau^{C I C}$ & -0.309 & -0.469 & -0.303 & -0.145 & -0.064 & -0.047 \\
\hline & {$[0.145]^{* *}$} & {$[0.699]$} & {$[0.232]$} & {$[0.099]$} & {$[0.080]$} & {$[0.096]$} \\
\hline$\tau_{w / c o v}^{C I C}$ & -0.333 & -0.432 & -0.535 & -0.202 & 0.038 & -0.064 \\
\hline & {$[0.183]^{*}$} & {$[0.884]$} & {$[0.356]$} & {$[0.141]$} & {$[0.148]$} & {$[0.166]$} \\
\hline $\mathrm{Mu}$ & eres & & & & & \\
\hline$\tau^{C I C}$ & -0.290 & -4.837 & -0.154 & -0.146 & -0.019 & -0.077 \\
\hline & {$[0.315]$} & {$[1.580]^{* * *}$} & {$[0.512]$} & {$[0.149]$} & {$[0.137]$} & {$[0.197]$} \\
\hline$\tau_{w / \text { cov }}^{C I C}$ & -0.237 & -2.955 & 0.307 & -0.054 & -0.142 & 0.169 \\
\hline & [0.338] & {$[1.200]^{* *}$} & {$[0.635]$} & {$[0.273]$} & {$[0.214]$} & {$[0.300]$} \\
\hline Hol & bres & & & & & \\
\hline$\tau^{C I C}$ & -0.014 & 0.288 & -0.237 & -0.047 & 0.054 & 0.149 \\
\hline & {$[0.200]$} & {$[0.683]$} & {$[0.321]$} & [0.139] & {$[0.134]$} & {$[0.147]$} \\
\hline$\tau_{w \nu \text { cov }}^{C I C}$ & -0.101 & 0.142 & -0.403 & -0.089 & 0.008 & 0.105 \\
\hline & {$[0.250]$} & {$[0.805]$} & {$[0.424]$} & {$[0.250]$} & {$[0.196]$} & {$[0.204]$} \\
\hline $\mathrm{Mu}$ & er y hombre & & & & & \\
\hline$\tau^{C I C}$ & -0.992 & -2.724 & -1.140 & -0.587 & -0.329 & -0.261 \\
\hline & {$[0.259]^{* * *}$} & {$[1.534]^{*}$} & {$[0.470]^{* *}$} & {$[0.246]^{* *}$} & {$[0.178]^{*}$} & {$[0.243]$} \\
\hline$\tau_{w / c o v}^{C I C}$ & -1.095 & -3.417 & -1.548 & -0.567 & -0.174 & -0.256 \\
\hline & {$[0.313]^{* * *}$} & {$[1.016]^{* * *}$} & {$[0.524]^{* * *}$} & {$[0.338]^{*}$} & {$[0.332]$} & {$[0.339]$} \\
\hline
\end{tabular}

Fuente: Estimación de los autores basada en la serie armonizada por Fundación ARU de encuestas de hogares (INE. 
Tabla 10: Efectos sobre el (log) del ingreso no laboral per-cápita del hogar, estimador Changes-in-Changes

\begin{tabular}{|c|c|c|c|c|c|c|}
\hline & $C I C_{\text {promedio }}$ & $C I C_{q 0,1}$ & $C I C_{q 0,2}$ & $C I C_{q 0,5}$ & $C I C_{q 0,8}$ & $C I C_{q 0,9}$ \\
\hline \multicolumn{7}{|c|}{ Bolivia } \\
\hline \multirow[t]{2}{*}{$\tau^{C I C}$} & 2.580 & 0.000 & 4.556 & 5.772 & 0.600 & 0.393 \\
\hline & {$[0.191]^{* * *}$} & {$[0.000]$} & {$[0.074]^{* * *}$} & {$[0.450]^{* * *}$} & {$[0.233]^{* *}$} & {$[0.192]^{* *}$} \\
\hline \multirow[t]{2}{*}{$\tau_{w / \operatorname{cov}}^{C I C}$} & 2.460 & 0.887 & 4.324 & 4.059 & 0.660 & 0.568 \\
\hline & {$[0.236]^{* * *}$} & {$[0.242]^{* * *}$} & {$[0.186]^{* * *}$} & {$[0.347]^{* * *}$} & {$[0.316]^{* *}$} & {$[0.278]^{* *}$} \\
\hline \multicolumn{7}{|c|}{ Mujeres } \\
\hline \multirow[t]{2}{*}{$\tau^{C I C}$} & 3.057 & 3.709 & 4.855 & 5.879 & 0.914 & 0.781 \\
\hline & {$[0.307]^{* * *}$} & {$[1.148]^{* * *}$} & {$[0.087]^{* * *}$} & {$[0.722]^{* * *}$} & {$[0.325]^{* * *}$} & {$[0.283]^{* * *}$} \\
\hline \multirow[t]{2}{*}{$\tau_{w / \operatorname{cov}}^{C I C}$} & 2.983 & 3.640 & 4.831 & 4.583 & 1.116 & 1.011 \\
\hline & {$[0.361]^{* * *}$} & {$[0.843]^{* * *}$} & {$[0.309]^{* * *}$} & {$[0.480]^{* * *}$} & {$[0.468]^{* *}$} & {$[0.473]^{* *}$} \\
\hline \multicolumn{7}{|c|}{ Hombres } \\
\hline \multirow[t]{2}{*}{$\tau^{C I C}$} & 1.946 & 0.000 & 2.457 & 5.477 & 0.111 & 0.174 \\
\hline & {$[0.309]^{* * *}$} & {$[0.000]$} & {$[1.143]^{* *}$} & {$[1.101]^{* * *}$} & {$[0.355]$} & {$[0.335]$} \\
\hline \multirow{2}{*}{$\tau_{w / \operatorname{cov}}^{C I C}$} & 1.896 & 0.467 & 2.156 & 3.779 & 0.002 & -0.139 \\
\hline & {$[0.353]^{* * *}$} & {$[0.233]^{* *}$} & {$[0.705]^{* * *}$} & {$[0.665]^{* * *}$} & {$[0.552]$} & {$[0.529]$} \\
\hline \multicolumn{7}{|c|}{ Mujer y hombre } \\
\hline \multirow[t]{2}{*}{$\tau^{C I C}$} & 2.925 & 0.000 & 4.858 & 5.998 & 0.149 & 0.503 \\
\hline & {$[0.447]^{* * *}$} & {$[1.173]$} & {$[0.161]^{* * *}$} & {$[0.981]^{* * *}$} & {$[0.621]$} & {$[0.425]$} \\
\hline \multirow{2}{*}{$\tau_{w / \operatorname{cov}}^{C I C}$} & 2.790 & 0.941 & 4.769 & 4.049 & 1.561 & 0.578 \\
\hline & {$[0.533]^{* * *}$} & {$[1.084]$} & {$[0.300]^{* * *}$} & {$[0.769]^{* * *}$} & [1.163] & {$[0.865]$} \\
\hline
\end{tabular}

Fuente: Estimación de los autores basada en la serie armonizada por Fundación ARU de encuestas de hogares (INE). 
Tabla 11: Efectos sobre las (log) transferencias totales hacia el hogar per-cápita, estimador Changes-in-Changes

\begin{tabular}{|c|c|c|c|c|c|c|}
\hline & $C I C_{\text {promedio }}$ & $C I C_{q 0,1}$ & $C I C_{q 0,2}$ & $C I C_{q 0,5}$ & $C I C_{q 0,8}$ & $C I C_{q 0,9}$ \\
\hline Boli & & & & & & \\
\hline$\tau^{C I C}$ & 0.088 & 0.000 & 0.000 & 0.000 & 0.427 & -0.220 \\
\hline & {$[0.215]$} & {$[0.000]$} & {$[0.000]$} & {$[0.000]$} & {$[0.683]$} & {$[0.295]$} \\
\hline$\tau_{w / \text { cov }}^{C I C}$ & 0.031 & 0.008 & -0.062 & -0.127 & 0.160 & 0.119 \\
\hline & {$[0.232]$} & {$[0.068]$} & {$[0.089]$} & {$[0.140]$} & {$[0.784]$} & {$[0.440]$} \\
\hline $\mathrm{Muj}$ & eres & & & & & \\
\hline$\tau^{C I C}$ & 0.633 & 0.000 & 0.000 & 0.000 & 0.508 & 0.258 \\
\hline & {$[0.393]$} & {$[0.000]$} & {$[0.000]$} & {$[0.000]$} & {$[0.692]$} & {$[0.460]$} \\
\hline$\tau_{w / \operatorname{cov}}^{C I C}$ & 0.300 & 0.037 & -0.040 & -0.132 & 0.393 & -0.179 \\
\hline & {$[0.415]$} & {$[0.214]$} & {$[0.241]$} & {$[0.182]$} & {$[0.862]$} & {$[0.453]$} \\
\hline Hon & bres & & & & & \\
\hline$\tau^{C I C}$ & -0.395 & 0.000 & 0.000 & 0.000 & -1.250 & -0.791 \\
\hline & {$[0.276]$} & {$[0.000]$} & {$[0.000]$} & {$[0.000]$} & {$[1.761]$} & [0.491] \\
\hline$\tau_{w / \operatorname{cov} v}^{C I C}$ & -0.358 & 0.052 & -0.077 & -0.112 & -1.112 & -0.586 \\
\hline & {$[0.263]$} & {$[0.089]$} & {$[0.105]$} & {$[0.109]$} & {$[1.386]$} & {$[0.564]$} \\
\hline Muj & er y hombre & & & & & \\
\hline$\tau^{C I C}$ & 0.466 & 0.000 & 0.000 & 0.000 & 5.004 & 0.168 \\
\hline & {$[0.472]$} & {$[0.000]$} & {$[0.000]$} & {$[0.000]$} & {$[1.852]^{* * *}$} & {$[2.068]$} \\
\hline$\tau_{w / \operatorname{cov} v}^{C I C}$ & 0.475 & -0.006 & 0.063 & 0.078 & 4.054 & -0.323 \\
\hline & [0.439] & {$[0.104]$} & {$[0.119]$} & {$[0.201]$} & {$[1.533]^{* * *}$} & {$[1.351]$} \\
\hline
\end{tabular}

Fuente: Estimación de los autores basada en la serie armonizada por Fundación ARU de encuestas de hogares (INE). 
Tabla 12: Estimados de Diff-in-Diff de indicadores de consumo del hogar

\begin{tabular}{|c|c|c|c|c|c|c|c|c|c|c|c|c|}
\hline & \multicolumn{3}{|c|}{ Bolivia } & \multicolumn{3}{|c|}{ Solo hombres IAI } & \multicolumn{3}{|c|}{ Solo mujeres IAI } & \multicolumn{3}{|c|}{ Mujer y hombre IAI } \\
\hline & {$[55,60)$} & {$[60,65)$} & DID & {$[55,60)$} & {$[60,65)$} & DID & {$[55,60)$} & {$[60,65)$} & DID & {$[55,60)$} & {$[60,65)$} & DID \\
\hline \multicolumn{13}{|c|}{ consumo del hogar per-cápita (log Bs. 2012 al mes) } \\
\hline \multirow[t]{2}{*}{$\mathrm{T}=0$} & 6.44 & 6.45 & & 6.39 & 6.51 & & 6.50 & 6.43 & & 6.41 & 6.33 & \\
\hline & {$[0.03]$} & {$[0.03]$} & 0.00 & {$[0.04]$} & {$[0.04]$} & -0.10 & {$[0.04]$} & {$[0.05]$} & 0.11 & {$[0.05]$} & {$[0.06]$} & 0.02 \\
\hline \multirow[t]{2}{*}{$\mathrm{T}=1$} & 6.65 & 6.66 & {$[0.05]$} & 6.68 & 6.70 & {$[0.08]$} & 6.66 & 6.70 & {$[0.09]$} & 6.55 & 6.51 & {$[0.11]$} \\
\hline & {$[0.02]$} & [0.02] & & [0.03] & [0.03] & & {$[0.03]$} & {$[0.04]$} & & {$[0.05]$} & {$[0.05]$} & \\
\hline \multicolumn{13}{|c|}{ consumo alimentario del hogar per-cápita (log Bs. 2012 al mes) } \\
\hline \multirow[t]{2}{*}{$\mathrm{T}=0$} & 5.97 & 5.95 & & 5.94 & 6.01 & & 6.04 & 5.92 & & 5.95 & 5.86 & \\
\hline & {$[0.03]$} & [0.03] & 0.04 & {$[0.04]$} & {$[0.04]$} & -0.06 & {$[0.04]$} & {$[0.05]$} & 0.13 & {$[0.05]$} & {$[0.06]$} & 0.09 \\
\hline \multirow[t]{2}{*}{$\mathrm{T}=1$} & 6.18 & 6.20 & {$[0.05]$} & 6.21 & 6.23 & {$[0.07]$} & 6.19 & 6.21 & {$[0.08]$} & 6.10 & 6.10 & {$[0.10]$} \\
\hline & {$[0.02]$} & {$[0.02]$} & & {$[0.03]$} & {$[0.03]$} & & {$[0.03]$} & {$[0.04]$} & & {$[0.04]$} & {$[0.05]$} & \\
\hline \multicolumn{13}{|c|}{ consumo no alimentario del hogar per-cápita (log Bs. 2012 al mes) } \\
\hline \multirow[t]{2}{*}{$\mathrm{T}=0$} & 4.10 & 4.07 & & 4.11 & 4.20 & & 4.14 & 3.99 & & 4.03 & 3.89 & \\
\hline & {$[0.04]$} & {$[0.05]$} & 0.01 & {$[0.07]$} & {$[0.08]$} & -0.07 & {$[0.07]$} & {$[0.09]$} & 0.13 & {$[0.09]$} & {$[0.12]$} & -0.02 \\
\hline \multirow[t]{2}{*}{$\mathrm{T}=1$} & 4.35 & 4.33 & {$[0.09]$} & 4.40 & 4.42 & [0.13] & 4.38 & 4.36 & {$[0.14]$} & 4.18 & 4.03 & [0.19] \\
\hline & {$[0.03]$} & {$[0.04]$} & & {$[0.05]$} & {$[0.06]$} & & {$[0.06]$} & {$[0.06]$} & & {$[0.08]$} & {$[0.09]$} & \\
\hline
\end{tabular}

Fuente: Estimación de los autores basada en la serie armonizada por Fundación ARU de encuestas de hogares (INE).

Notas: Errores estándar entre corchetes (ver nota de figura 7). IAI=en el intervalo de edad (in age interval).

Figura 4: Función de distribución acumulada empírica del (log) consumo total del hogar percápita
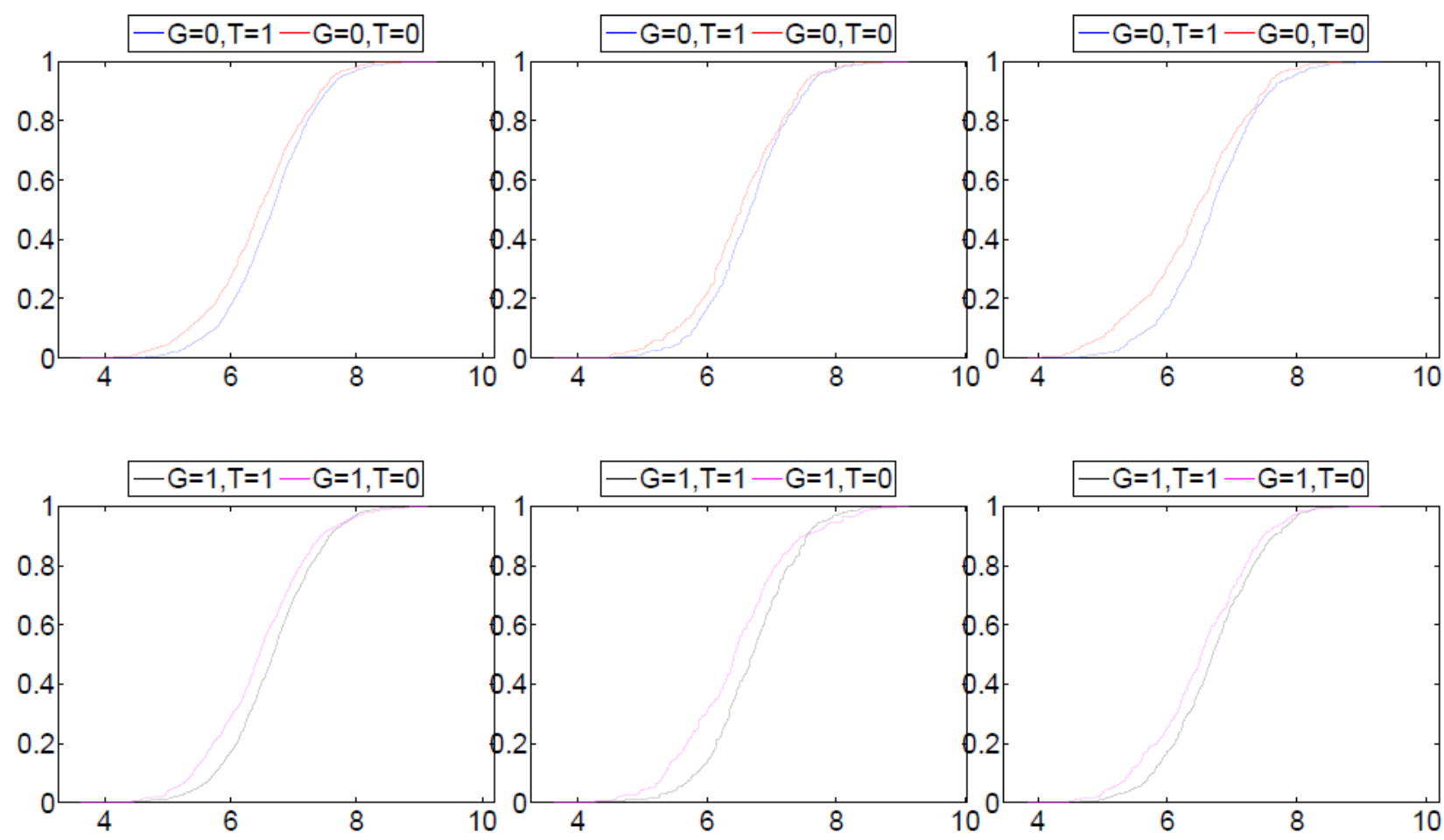

(a) Bolivia

(b) Mujeres

(c) Hombres

Fuente: Estimación de los autores basada en la serie armonizada por Fundación ARU de encuestas de hogares (INE). 
Tabla 13: Efectos sobre el (log) del consumo total del hogar per-cápita, estimador Changesin-Changes

\begin{tabular}{|c|c|c|c|c|c|c|}
\hline & $C I C_{\text {promedio }}$ & $C I C_{q 0,1}$ & $C I C_{q 0,2}$ & $C I C_{q 0,5}$ & $C I C_{q 0,8}$ & $C I C_{q 0,9}$ \\
\hline \multicolumn{7}{|c|}{ Bolivia } \\
\hline \multirow[t]{2}{*}{$\tau^{C I C}$} & -0.007 & -0.073 & 0.104 & -0.003 & 0.060 & 0.018 \\
\hline & {$[0.050]$} & {$[0.082]$} & {$[0.070]$} & {$[0.055]$} & {$[0.071]$} & [0.089] \\
\hline \multirow{2}{*}{$\tau_{w / \operatorname{cov}}^{C I C}$} & -0.017 & 0.048 & 0.005 & -0.018 & 0.005 & 0.066 \\
\hline & {$[0.083]$} & {$[0.144]$} & {$[0.114]$} & {$[0.100]$} & {$[0.131]$} & {$[0.114]$} \\
\hline \multicolumn{7}{|c|}{ Mujeres } \\
\hline \multirow[t]{2}{*}{$\tau^{C I C}$} & 0.089 & 0.172 & 0.239 & 0.118 & 0.173 & -0.050 \\
\hline & {$[0.082]$} & {$[0.148]$} & {$[0.098]^{* *}$} & {$[0.092]$} & {$[0.129]$} & {$[0.212]$} \\
\hline \multirow{2}{*}{$\tau_{w / c o v}^{C I C}$} & 0.148 & 0.417 & 0.342 & 0.104 & 0.128 & -0.165 \\
\hline & {$[0.140]$} & {$[0.269]$} & {$[0.176]^{*}$} & {$[0.172]$} & {$[0.244]$} & {$[0.244]$} \\
\hline \multicolumn{7}{|c|}{ Hombres } \\
\hline \multirow[t]{2}{*}{$\tau^{C I C}$} & -0.075 & -0.172 & -0.083 & -0.032 & 0.018 & 0.064 \\
\hline & {$[0.068]$} & {$[0.096]^{*}$} & {$[0.114]$} & {$[0.065]$} & {$[0.094]$} & {$[0.180]$} \\
\hline \multirow[t]{2}{*}{$\tau_{w / \operatorname{cov}}^{C I C}$} & -0.126 & -0.291 & -0.179 & -0.160 & -0.099 & 0.101 \\
\hline & [0.118] & {$[0.226]$} & {$[0.160]$} & {$[0.149]$} & {$[0.177]$} & [0.183] \\
\hline \multicolumn{7}{|c|}{ Mujer y hombre } \\
\hline \multirow[t]{2}{*}{$\tau^{C I C}$} & 0.031 & 0.018 & -0.036 & 0.008 & -0.018 & 0.166 \\
\hline & {$[0.110]$} & {$[0.263]$} & {$[0.183]$} & {$[0.144]$} & {$[0.155]$} & {$[0.180]$} \\
\hline \multirow[t]{2}{*}{$\tau_{w / c o v}^{C I C}$} & -0.021 & -0.044 & -0.093 & 0.001 & -0.011 & -0.017 \\
\hline & {$[0.183]$} & {$[0.376]$} & {$[0.248]$} & {$[0.209]$} & {$[0.233]$} & {$[0.319]$} \\
\hline
\end{tabular}

Fuente: Estimación de los autores basada en la serie armonizada por Fundación ARU de encuestas de hogares (INE). 
Tabla 14: Efectos sobre el (log) del consumo alimentario del hogar per-cápita, estimador Changes-in-Changes

\begin{tabular}{|c|c|c|c|c|c|c|}
\hline & $C I C_{\text {promedio }}$ & $C I C_{q 0,1}$ & $C I C_{q 0,2}$ & $C I C_{q 0,5}$ & $C I C_{q 0,8}$ & $C I C_{q 0,9}$ \\
\hline \multicolumn{7}{|c|}{ Bolivia } \\
\hline \multirow[t]{2}{*}{$\tau^{C I C}$} & 0.036 & 0.055 & 0.030 & 0.048 & 0.080 & 0.071 \\
\hline & {$[0.051]$} & {$[0.100]$} & {$[0.075]$} & {$[0.060]$} & {$[0.073]$} & {$[0.073]$} \\
\hline \multirow[t]{2}{*}{$\tau_{w / \operatorname{cov}}^{C I C}$} & 0.031 & -0.001 & 0.096 & -0.002 & 0.060 & 0.134 \\
\hline & {$[0.070]$} & {$[0.119]$} & {$[0.081]$} & {$[0.086]$} & {$[0.080]$} & [0.131] \\
\hline \multicolumn{7}{|c|}{ Mujeres } \\
\hline \multirow[t]{2}{*}{$\tau^{C I C}$} & 0.116 & 0.066 & 0.069 & 0.175 & 0.162 & 0.157 \\
\hline & {$[0.073]$} & {$[0.131]$} & {$[0.093]$} & {$[0.087]^{* *}$} & {$[0.126]$} & {$[0.172]$} \\
\hline \multirow{2}{*}{$\tau_{w / \operatorname{cov}}^{C I C}$} & 0.146 & 0.252 & 0.328 & 0.154 & 0.209 & 0.002 \\
\hline & {$[0.115]$} & {$[0.264]$} & {$[0.149]^{* *}$} & [0.138] & {$[0.108]^{*}$} & {$[0.202]$} \\
\hline \multicolumn{7}{|c|}{ Hombres } \\
\hline \multirow[t]{2}{*}{$\tau^{C I C}$} & -0.043 & -0.069 & -0.125 & -0.048 & -0.060 & -0.033 \\
\hline & {$[0.061]$} & {$[0.130]$} & {$[0.113]$} & {$[0.087]$} & {$[0.103]$} & {$[0.110]$} \\
\hline \multirow{2}{*}{$\tau_{w / c o v}^{C I C}$} & -0.075 & -0.220 & -0.200 & -0.098 & 0.035 & 0.036 \\
\hline & {$[0.101]$} & {$[0.168]$} & {$[0.161]$} & {$[0.109]$} & {$[0.095]$} & {$[0.206]$} \\
\hline \multicolumn{7}{|c|}{ Mujer y hombre } \\
\hline \multirow[t]{2}{*}{$\tau^{C I C}$} & 0.076 & 0.101 & 0.090 & 0.066 & 0.144 & 0.236 \\
\hline & {$[0.103]$} & {$[0.196]$} & {$[0.195]$} & {$[0.100]$} & {$[0.144]$} & {$[0.138]^{*}$} \\
\hline \multirow{2}{*}{$\tau_{w / \operatorname{cov}}^{C I C}$} & 0.066 & 0.180 & -0.069 & 0.035 & 0.219 & 0.201 \\
\hline & {$[0.157]$} & {$[0.273]$} & {$[0.335]$} & {$[0.163]$} & {$[0.224]$} & {$[0.222]$} \\
\hline
\end{tabular}

Fuente: Estimación de los autores basada en la serie armonizada por Fundación ARU de encuestas de hogares (INE). 
Tabla 15: Efectos sobre el (log) consumo no alimentario per-cápita, estimador Changes-inChanges

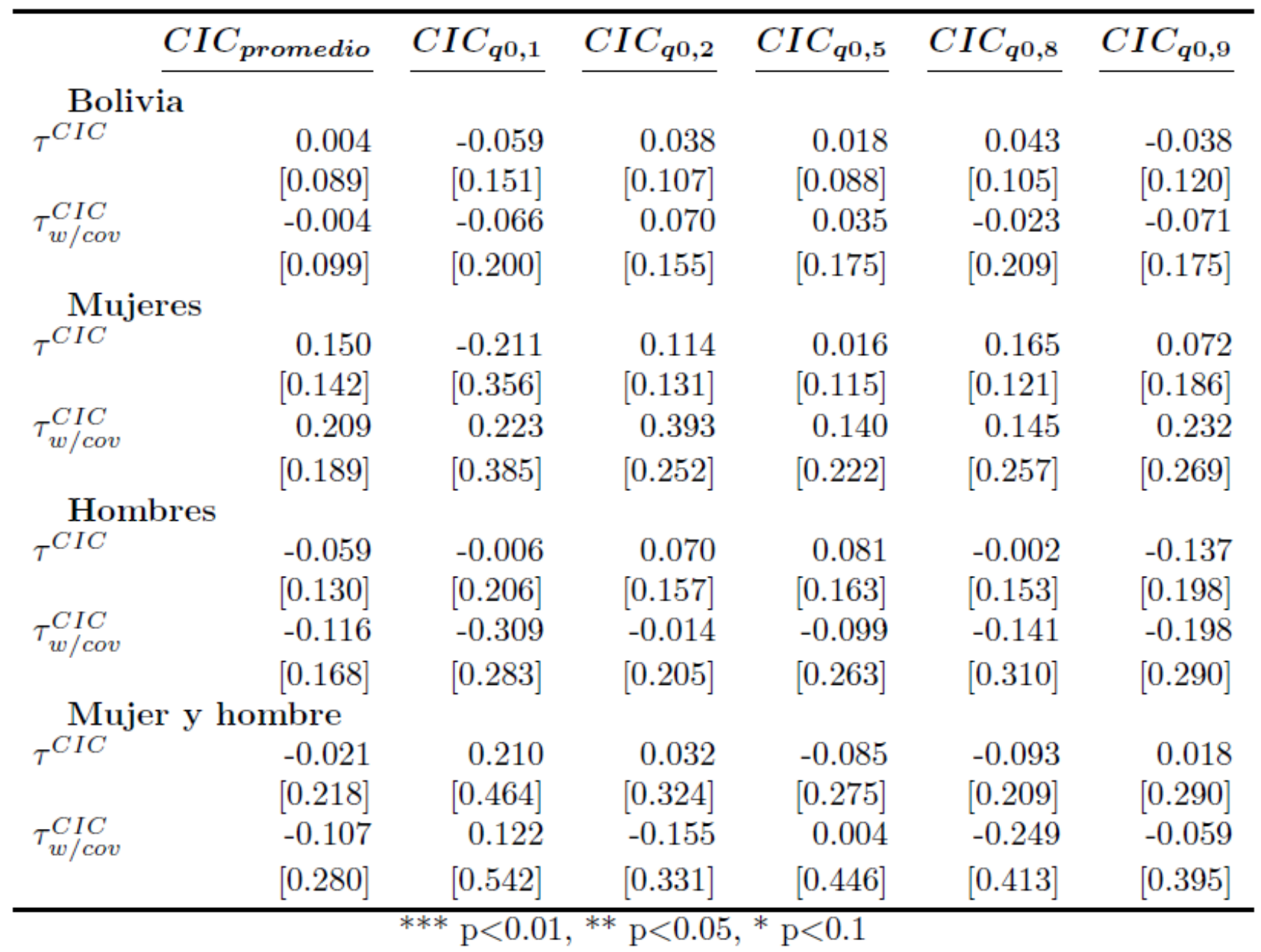

Fuente: Estimación de los autores basada en la serie armonizada por Fundación ARU de encuestas de hogares (INE). 
Tabla 16: Estimados de Diff-in-Diff de indicadores de inversión

\begin{tabular}{|c|c|c|c|c|c|c|c|c|c|c|c|c|}
\hline & \multicolumn{3}{|c|}{ Bolivia } & \multicolumn{3}{|c|}{ Solo hombres IAI } & \multicolumn{3}{|c|}{ Solo mujeres IAI } & \multicolumn{3}{|c|}{ Mujer y hombre IAI } \\
\hline & {$[55,60)$} & {$[60,65)$} & DID & {$[55,60)$} & {$[60,65)$} & DID & {$[55,60)$} & {$[60,65)$} & DID & {$[55,60)$} & {$[60,65)$} & DID \\
\hline \multicolumn{13}{|c|}{ Inversión en educación (log Bs. 2012 al mes) } \\
\hline \multirow[t]{2}{*}{$\mathrm{T}=0$} & 1.17 & 0.32 & & 1.51 & 0.71 & & 0.75 & 0.09 & & 1.14 & -0.29 & \\
\hline & {$[0.12]$} & {$[0.13]$} & -0.02 & {$[0.17]$} & {$[0.20]$} & -0.01 & {$[0.20]$} & {$[0.22]$} & 0.02 & {$[0.26]$} & {$[0.31]$} & -0.07 \\
\hline \multirow[t]{2}{*}{$\mathrm{T}=1$} & 0.98 & 0.12 & {$[0.24]$} & 1.26 & 0.46 & {$[0.34]$} & 0.63 & -0.00 & {$[0.40]$} & 0.94 & -0.57 & {$[0.53]$} \\
\hline & {$[0.10]$} & {$[0.11]$} & & {$[0.15]$} & {$[0.17]$} & & {$[0.18]$} & {$[0.20]$} & & {$[0.25]$} & {$[0.25]$} & \\
\hline \multicolumn{13}{|c|}{ Inversión en educación (log Bs. 2012 al mes) } \\
\hline \multirow[t]{2}{*}{$\mathrm{T}=0$} & -0.26 & -0.22 & & -0.40 & -0.36 & & -0.06 & 0.00 & & -0.32 & -0.29 & \\
\hline & {$[0.10]$} & {$[0.12]$} & 0.03 & {$[0.15]$} & {$[0.17]$} & -0.02 & {$[0.18]$} & {$[0.19]$} & -0.07 & {$[0.23]$} & {$[0.28]$} & 0.36 \\
\hline \multirow[t]{2}{*}{$\mathrm{T}=1$} & -0.18 & -0.10 & {$[0.21]$} & -0.20 & -0.18 & {$[0.30]$} & -0.06 & -0.07 & {$[0.36]$} & -0.38 & 0.02 & {$[0.48]$} \\
\hline & {$[0.09]$} & {$[0.10]$} & & {$[0.13]$} & {$[0.15]$} & & {$[0.16]$} & {$[0.18]$} & & {$[0.22]$} & {$[0.24]$} & \\
\hline \multicolumn{13}{|c|}{ Inversión en durables (el año pasado) (log Bs. 2012 al mes) } \\
\hline \multirow[t]{2}{*}{$\mathrm{T}=0$} & 1.78 & 1.36 & & 1.84 & 1.44 & & 1.68 & 1.24 & & 1.84 & 1.42 & \\
\hline & [0.09] & {$[0.10]$} & 0.09 & {$[0.14]$} & {$[0.15]$} & -0.05 & {$[0.15]$} & {$[0.16]$} & 0.32 & {$[0.22]$} & {$[0.23]$} & 0.01 \\
\hline \multirow[t]{2}{*}{$\mathrm{T}=1$} & 1.66 & 1.33 & {$[0.18]$} & 1.80 & 1.35 & {$[0.27]$} & 1.49 & 1.36 & {$[0.30]$} & 1.63 & 1.21 & {$[0.42]$} \\
\hline & {$[0.08]$} & {$[0.08]$} & & {$[0.12]$} & {$[0.12]$} & & {$[0.14]$} & {$[0.15]$} & & {$[0.19]$} & {$[0.18]$} & \\
\hline \multicolumn{13}{|c|}{ Inversión en la vivienda (log Bs. 2012 al mes) } \\
\hline \multirow[t]{2}{*}{$\mathrm{T}=0$} & -0.32 & -0.57 & & -0.45 & -0.64 & & -0.31 & -0.47 & & -0.01 & -0.59 & \\
\hline & {$[0.08]$} & {$[0.08]$} & 0.25 & {$[0.12]$} & {$[0.11]$} & 0.09 & {$[0.14]$} & [0.13] & 0.38 & {$[0.20]$} & {$[0.17]$} & 0.40 \\
\hline \multirow[t]{2}{*}{$\mathrm{T}=1$} & -0.40 & -0.41 & {$[0.15]^{*}$} & -0.37 & -0.47 & {$[0.21]$} & -0.58 & -0.36 & {$[0.25]$} & -0.15 & -0.34 & {$[0.36]$} \\
\hline & {$[0.07]$} & {$[0.07]$} & & {$[0.09]$} & {$[0.10]$} & & {$[0.10]$} & [0.13] & & {$[0.19]$} & {$[0.17]$} & \\
\hline
\end{tabular}

Fuente: Estimación de los autores basada en la serie armonizada por Fundación ARU de encuestas de hogares (INE).

Notas: Errores estándar entre corchetes (ver nota de figura 7). IAI=en el intervalo de edad (in age interval). 
Tabla 17: Efectos sobre la inversión en educación (log), estimador Changes-in-Changes

\begin{tabular}{|c|c|c|c|c|c|c|}
\hline & $C I C_{\text {promedio }}$ & $C I C_{q 0,1}$ & $C I C_{q 0,2}$ & $C I C_{q 0,5}$ & $C I C_{q 0,8}$ & $C I C_{q 0,9}$ \\
\hline \multicolumn{7}{|c|}{ Bolivia } \\
\hline \multirow{2}{*}{$\tau^{C I C}$} & -0.005 & 0.000 & 0.000 & 0.000 & -0.033 & 0.044 \\
\hline & {$[0.221]$} & {$[0.000]$} & {$[0.000]$} & [2.388] & {$[0.183]$} & {$[0.168]$} \\
\hline \multirow{2}{*}{$\tau_{w / \operatorname{cov}}^{C I C}$} & 0.068 & 0.085 & -0.043 & -0.017 & -0.036 & 0.160 \\
\hline & {$[0.353]$} & {$[0.113]$} & {$[0.195]$} & {$[1.587]$} & {$[0.386]$} & {$[0.375]$} \\
\hline \multicolumn{7}{|c|}{ Mujeres } \\
\hline \multirow[t]{2}{*}{$\tau^{C I C}$} & 0.116 & 0.000 & 0.000 & 0.000 & -0.085 & 0.191 \\
\hline & {$[0.416]$} & {$[0.000]$} & {$[0.000]$} & {$[2.317]$} & {$[0.322]$} & {$[0.250]$} \\
\hline \multirow{2}{*}{$\tau_{w / c o v}^{C I C}$} & 0.400 & 0.165 & 0.123 & 0.332 & 0.910 & -0.048 \\
\hline & [0.609] & {$[0.163]$} & {$[0.346]$} & {$[1.787]$} & {$[0.705]$} & {$[0.885]$} \\
\hline \multicolumn{7}{|c|}{ Hombres } \\
\hline \multirow[t]{2}{*}{$\tau^{C I C}$} & -0.131 & 0.000 & 0.000 & -0.770 & 0.070 & -0.011 \\
\hline & {$[0.295]$} & {$[0.000]$} & {$[0.000]$} & {$[2.857]$} & {$[0.253]$} & {$[0.295]$} \\
\hline \multirow{2}{*}{$\tau_{w / c o v}^{C I C}$} & -0.065 & -0.132 & 0.021 & -0.229 & -0.309 & 0.527 \\
\hline & {$[0.535]$} & {$[0.222]$} & [0.398] & [2.493] & {$[0.483]$} & {$[0.547]$} \\
\hline \multicolumn{7}{|c|}{ Mujer y hombre } \\
\hline \multirow[t]{2}{*}{$\tau^{C I C}$} & 0.055 & 0.000 & 0.000 & 0.000 & -0.618 & -0.161 \\
\hline & {$[0.514]$} & {$[0.000]$} & {$[0.000]$} & {$[1.166]$} & {$[0.431]$} & {$[0.394]$} \\
\hline \multirow{2}{*}{$\tau_{w / \operatorname{cov}}^{C I C}$} & -0.251 & -0.129 & 0.119 & -0.044 & -0.782 & -0.477 \\
\hline & {$[0.734]$} & {$[0.236]$} & {$[0.432]$} & {$[1.027]$} & [1.148] & {$[0.880]$} \\
\hline
\end{tabular}

Fuente: Estimación de los autores basada en la serie armonizada por Fundación ARU de encuestas de hogares (INE). 
Tabla 18: Efectos sobre la inversión en salud (log), estimador Changes-in-Changes

\begin{tabular}{|c|c|c|c|c|c|c|}
\hline & $C I C_{\text {promedio }}$ & $C I C_{q 0,1}$ & $C I C_{q 0,2}$ & $C I C_{q 0,5}$ & $C I C_{q 0,8}$ & $C I C_{q 0,9}$ \\
\hline \multicolumn{7}{|c|}{ Bolivia } \\
\hline \multirow[t]{2}{*}{$\tau^{C I C}$} & 0.040 & 0.000 & 0.000 & 0.182 & -0.292 & -0.014 \\
\hline & {$[0.221]$} & {$[0.000]$} & {$[0.000]$} & {$[1.242]$} & {$[0.182]$} & [0.213] \\
\hline \multirow[t]{2}{*}{$\tau_{w / \operatorname{cov}}^{C I C}$} & 0.025 & 0.027 & 0.027 & 0.167 & 0.248 & -0.123 \\
\hline & {$[0.245]$} & {$[0.148]$} & {$[0.155]$} & {$[0.737]$} & {$[0.346]$} & {$[0.277]$} \\
\hline \multicolumn{7}{|c|}{ Mujeres } \\
\hline \multirow[t]{2}{*}{$\tau^{C I C}$} & -0.069 & 0.000 & 0.000 & 0.267 & -0.292 & 0.306 \\
\hline & {$[0.380]$} & {$[0.000]$} & {$[0.000]$} & {$[2.088]$} & {$[0.331]$} & {$[0.311]$} \\
\hline \multirow{2}{*}{$\tau_{w / \operatorname{cov}}^{C I C}$} & -0.006 & 0.480 & -0.015 & -0.115 & 0.079 & 0.283 \\
\hline & {$[0.417]$} & {$[0.322]$} & {$[0.334]$} & {$[1.144]$} & {$[0.601]$} & {$[0.351]$} \\
\hline \multicolumn{7}{|c|}{ Hombres } \\
\hline \multirow[t]{2}{*}{$\tau^{C I C}$} & -0.020 & 0.000 & 0.000 & 0.046 & -0.318 & 0.023 \\
\hline & {$[0.320]$} & {$[0.000]$} & {$[0.000]$} & [1.388] & {$[0.265]$} & [0.336] \\
\hline \multirow{2}{*}{$\tau_{w / \operatorname{cov}}^{C I C}$} & -0.094 & -0.062 & -0.040 & 0.285 & -0.223 & 0.007 \\
\hline & {$[0.344]$} & {$[0.192]$} & {$[0.282]$} & {$[0.949]$} & {$[0.431]$} & {$[0.464]$} \\
\hline \multicolumn{7}{|c|}{ Mujer y hombre } \\
\hline \multirow[t]{2}{*}{$\tau^{C I C}$} & 0.354 & 0.000 & 0.000 & 4.449 & -0.554 & -0.608 \\
\hline & {$[0.487]$} & {$[0.000]$} & {$[0.000]$} & {$[1.467]^{* * *}$} & {$[0.628]$} & {$[0.711]$} \\
\hline \multirow[t]{2}{*}{$\tau_{w / c o v}^{C I C}$} & 0.392 & -0.115 & -0.018 & 2.345 & 0.745 & -0.504 \\
\hline & {$[0.542]$} & {$[0.275]$} & {$[0.318]$} & {$[1.404]^{*}$} & {$[0.752]$} & {$[0.791]$} \\
\hline
\end{tabular}

Fuente: Estimación de los autores basada en la serie armonizada por Fundación ARU de encuestas de hogares (INE). 
Tabla 19: Efectos sobre la adquisición de bienes durables adquiridos el último año (log), estimador Changes-in-Changes

\begin{tabular}{|c|c|c|c|c|c|c|}
\hline & $C I C_{\text {promedio }}$ & $C I C_{q 0,1}$ & $C I C_{q 0,2}$ & $C I C_{q 0,5}$ & $C I C_{q 0,8}$ & $C I C_{q 0,9}$ \\
\hline \multicolumn{7}{|c|}{ Bolivia } \\
\hline \multirow[t]{2}{*}{$\tau^{C I C}$} & 0.072 & 0.000 & 0.000 & 0.000 & 0.339 & -0.006 \\
\hline & {$[0.154]$} & {$[0.000]$} & {$[0.000]$} & {$[0.000]$} & {$[0.257]$} & {$[0.278]$} \\
\hline \multirow{2}{*}{$\tau_{w / c o v}^{C I C}$} & 0.136 & 0.193 & 0.131 & 0.007 & 0.398 & 0.013 \\
\hline & {$[0.188]$} & {$[0.108]^{*}$} & {$[0.114]$} & {$[0.165]$} & {$[0.377]$} & {$[0.327]$} \\
\hline \multicolumn{7}{|c|}{ Mujeres } \\
\hline \multirow[t]{2}{*}{$\tau^{C I C}$} & 0.219 & 0.000 & 0.000 & 0.000 & 0.232 & 0.410 \\
\hline & {$[0.262]$} & {$[0.000]$} & {$[0.000]$} & {$[0.000]$} & {$[0.460]$} & {$[0.436]$} \\
\hline \multirow[t]{2}{*}{$\tau_{w / c o v}^{C I C}$} & 0.400 & 0.267 & 0.318 & 0.301 & 0.464 & 0.404 \\
\hline & {$[0.311]$} & {$[0.166]$} & {$[0.182]^{*}$} & {$[0.228]$} & {$[0.673]$} & {$[0.544]$} \\
\hline \multicolumn{7}{|c|}{ Hombres } \\
\hline \multirow[t]{2}{*}{$\tau^{C I C}$} & -0.022 & 0.000 & 0.000 & 0.000 & 0.292 & -0.111 \\
\hline & {$[0.246]$} & {$[0.000]$} & {$[0.000]$} & {$[0.000]$} & {$[0.370]$} & {$[0.432]$} \\
\hline \multirow[t]{2}{*}{$\tau_{w / c o v}^{C I C}$} & -0.076 & -0.047 & 0.027 & -0.163 & 0.141 & -0.196 \\
\hline & {$[0.277]$} & {$[0.134]$} & {$[0.179]$} & {$[0.269]$} & {$[0.469]$} & {$[0.480]$} \\
\hline \multicolumn{7}{|c|}{ Mujer y hombre } \\
\hline$\tau^{C I C}$ & 0.023 & 0.000 & 0.000 & 0.000 & 0.274 & 0.149 \\
\hline \multirow{3}{*}{$\tau_{w / c o v}^{C I C}$} & {$[0.359]$} & {$[0.000]$} & {$[0.000]$} & {$[0.000]$} & {$[0.704]$} & {$[0.735]$} \\
\hline & 0.096 & 0.313 & 0.150 & -0.154 & 0.341 & 0.337 \\
\hline & {$[0.425]$} & {$[0.217]$} & {$[0.212]$} & {$[0.383]$} & [1.065] & {$[0.927]$} \\
\hline
\end{tabular}

Fuente: Estimación de los autores basada en la serie armonizada por Fundación ARU de encuestas de hogares (INE). 
Tabla 20: Efectos sobre la inversión en la vivienda (log), estimador Changes-in-Changes

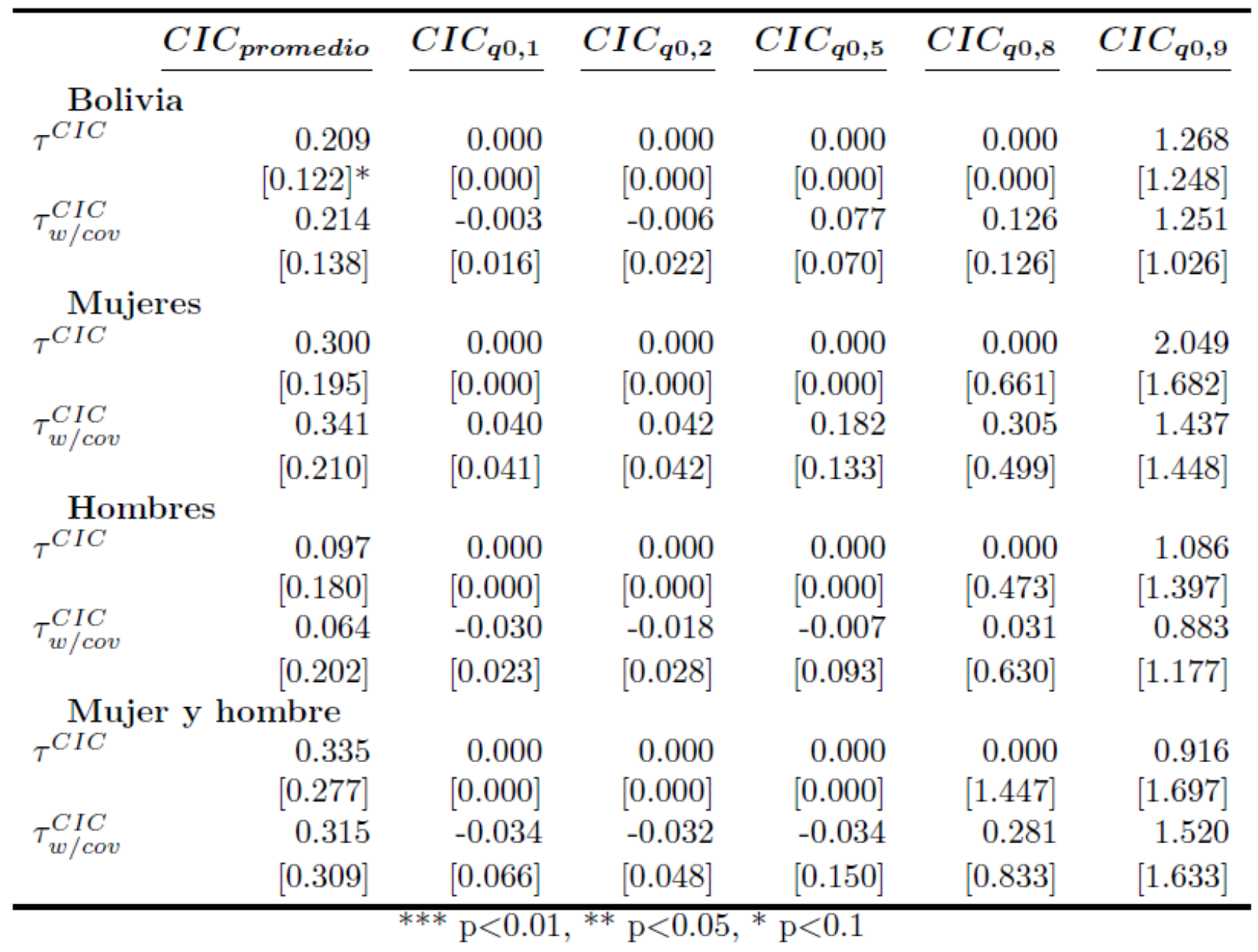

Fuente: Estimación de los autores basada en la serie armonizada por Fundación ARU de encuestas de hogares (INE). 
Tabla 21: Estimados de Diff-in-Diff de indicadores de ahorro

\begin{tabular}{|c|c|c|c|c|c|c|c|c|c|c|c|c|}
\hline & \multicolumn{3}{|c|}{ Bolivia } & \multicolumn{3}{|c|}{ Solo hombres IAI } & \multicolumn{3}{|c|}{ Solo mujeres IAI } & \multicolumn{3}{|c|}{ Mujer y hombre IAI } \\
\hline & {$[55,60)$} & {$[60,65)$} & DID & {$[55,60)$} & {$[60,65)$} & DID & {$[55,60)$} & {$[60,65)$} & DID & {$[55,60)$} & {$[60,65)$} & DID \\
\hline \multicolumn{13}{|c|}{ Ahorro del hogar per-cápita: (y-c) } \\
\hline \multirow[t]{2}{*}{$\mathrm{T}=0$} & 327.90 & 174.84 & & 515.13 & 223.97 & & 115.82 & 76.87 & & 280.16 & 247.72 & \\
\hline & {$[51.61]$} & {$[44.27]$} & 114.97 & [96.75] & {$[62.57]$} & 257.42 & {$[56.60]$} & {$[83.36]$} & 44.22 & {$[91.47]$} & {$[79.32]$} & -112.21 \\
\hline \multirow{2}{*}{$\mathrm{T}=1$} & 195.09 & 157.01 & {$[86.29]$} & 267.35 & 233.61 & {$[147.87]^{*}$} & 110.44 & 115.72 & {$[127.61]$} & 176.22 & 31.56 & [141.96] \\
\hline & {$[39.56]$} & {$[35.47]$} & & {$[72.00]$} & {$[58.35]$} & & {$[52.87]$} & {$[57.75]$} & & {$[56.72]$} & {$[47.70]$} & \\
\hline \multicolumn{13}{|c|}{ Tasa de ahorro $y:(y-c) / y$} \\
\hline \multirow[t]{2}{*}{$\mathrm{T}=0$} & -1.05 & -1.77 & & -1.05 & -1.53 & & -0.95 & -2.20 & & -1.22 & -1.53 & \\
\hline & {$[0.22]$} & {$[0.31]$} & 1.10 & {$[0.43]$} & {$[0.48]$} & 0.99 & {$[0.18]$} & {$[0.52]$} & 1.69 & {$[0.35]$} & {$[0.64]$} & 0.17 \\
\hline \multirow[t]{2}{*}{$\mathrm{T}=1$} & -1.08 & -0.70 & {$[0.42]^{* * * *}$} & -1.08 & -0.56 & {$[0.68]$} & -1.03 & -0.59 & {$[0.60]^{* * * *}$} & -1.17 & -1.31 & {$[0.96]$} \\
\hline & {$[0.15]$} & {$[0.09]$} & & {$[0.19]$} & {$[0.09]$} & & {$[0.20]$} & {$[0.10]$} & & {$[0.51]$} & {$[0.38]$} & \\
\hline \multicolumn{13}{|c|}{ Tasa de ahorro ${ }_{c}:(\mathrm{y}-\mathrm{c}) / \mathrm{c}$} \\
\hline \multirow[t]{2}{*}{$\mathrm{T}=0$} & 0.29 & 0.12 & & 0.46 & 0.15 & & 0.11 & 0.07 & & 0.23 & 0.15 & \\
\hline & {$[0.04]$} & {$[0.03]$} & 0.13 & {$[0.08]$} & {$[0.05]$} & 0.24 & {$[0.05]$} & {$[0.06]$} & 0.12 & {$[0.11]$} & {$[0.07]$} & -0.13 \\
\hline \multirow[t]{2}{*}{$\mathrm{T}=1$} & 0.21 & 0.18 & {$[0.07]^{*}$} & 0.28 & 0.21 & {$[0.12]^{* *}$} & 0.11 & 0.19 & {$[0.10]$} & 0.26 & 0.05 & {$[0.16]$} \\
\hline & {$[0.03]$} & {$[0.03]$} & & {$[0.06]$} & {$[0.04]$} & & {$[0.04]$} & {$[0.05]$} & & {$[0.07]$} & {$[0.06]$} & \\
\hline \multicolumn{13}{|c|}{ Tasa de ahorro aproximada $(\log y-\log c)$} \\
\hline \multirow[t]{2}{*}{$\mathrm{T}=0$} & -0.11 & -0.26 & & -0.00 & -0.22 & & -0.19 & -0.34 & & -0.22 & -0.21 & \\
\hline & {$[0.03]$} & {$[0.04]$} & 0.17 & {$[0.05]$} & {$[0.05]$} & 0.23 & {$[0.05]$} & {$[0.06]$} & 0.25 & {$[0.08]$} & {$[0.09]$} & -0.14 \\
\hline \multirow[t]{2}{*}{$\mathrm{T}=1$} & -0.14 & -0.11 & {$[0.06]^{* * * *}$} & -0.11 & -0.09 & {$[0.09]^{* * *}$} & -0.20 & -0.10 & {$[0.10]^{* *}$} & -0.10 & -0.23 & {$[0.15]$} \\
\hline & {$[0.03]$} & {$[0.03]$} & & {$[0.04]$} & {$[0.04]$} & & {$[0.04]$} & {$[0.04]$} & & {$[0.06]$} & {$[0.06]$} & \\
\hline
\end{tabular}

Fuente: Estimación de los autores basada en la serie armonizada por Fundación ARU de encuestas de hogares (INE).

Notas: Errores estándar entre corchetes (ver nota de figura 7). IAI=en el intervalo de edad (in age interval). 
Tabla 22: Efectos sobre el ahorro del hogar per-cápita (Bs.), estimador Changes-in-Changes

\begin{tabular}{|c|c|c|c|c|c|c|}
\hline & $C I C_{\text {promedio }}$ & $C I C_{q 0,1}$ & $C I C_{q 0,2}$ & $C I C_{q 0,5}$ & $C I C_{q 0,8}$ & $C I C_{q 0,9}$ \\
\hline \multicolumn{7}{|c|}{ Bolivia } \\
\hline \multirow[t]{2}{*}{$\tau^{C I C}$} & 105.430 & 96.122 & 13.964 & 68.058 & 67.159 & 159.030 \\
\hline & {$[75.127]$} & {$[86.366]$} & {$[50.272]$} & {$[28.037]^{* *}$} & {$[102.090]$} & [172.340] \\
\hline \multirow{2}{*}{$\tau_{w / \text { cov }}^{C I C}$} & 86.307 & 64.167 & 71.803 & 51.242 & 135.330 & 227.270 \\
\hline & {$[90.483]$} & {$[84.724]$} & {$[50.909]$} & {$[57.748]$} & {$[143.050]$} & {$[140.770]$} \\
\hline \multicolumn{7}{|c|}{ Mujeres } \\
\hline \multirow[t]{2}{*}{$\tau^{C I C}$} & 90.146 & -59.229 & 5.971 & 118.210 & 162.990 & 209.820 \\
\hline & {$[125.700]$} & {$[161.590]$} & {$[91.207]$} & {$[48.020]^{* *}$} & {$[123.820]$} & {$[346.270]$} \\
\hline \multirow{2}{*}{$\tau_{w / \operatorname{cov}}^{C I C}$} & 115.760 & -66.394 & 14.589 & 166.510 & 230.650 & 210.470 \\
\hline & {$[134.300]$} & {$[116.400]$} & {$[84.548]$} & {$[89.190]^{*}$} & {$[168.210]$} & {$[570.140]$} \\
\hline \multicolumn{7}{|c|}{ Hombres } \\
\hline \multirow[t]{2}{*}{$\tau^{C I C}$} & 219.110 & 301.450 & 64.440 & 97.342 & 72.304 & 260.340 \\
\hline & {$[129.080]^{*}$} & {$[160.580]^{*}$} & {$[84.820]$} & {$[46.704]^{* *}$} & {$[129.760]$} & [186.930] \\
\hline \multirow{2}{*}{$\tau_{w / \operatorname{cov}}^{C I C}$} & 176.920 & 120.930 & 155.310 & 31.495 & 212.440 & 401.210 \\
\hline & {$[144.210]$} & {$[127.420]$} & {$[86.138]^{*}$} & {$[85.544]$} & {$[182.800]$} & {$[243.150]^{*}$} \\
\hline \multicolumn{7}{|c|}{ Mujer y hombre } \\
\hline \multirow[t]{2}{*}{$\tau^{C I C}$} & -179.950 & -50.954 & -58.849 & -180.940 & -298.040 & -257.770 \\
\hline & {$[119.230]$} & {$[182.470]$} & {$[102.790]$} & {$[116.120]$} & {$[203.770]$} & [294.630] \\
\hline \multirow{2}{*}{$\tau_{w / \operatorname{cov}}^{C I C}$} & -175.730 & -116.600 & -35.186 & -183.520 & -441.590 & -387.160 \\
\hline & {$[132.580]$} & {$[130.920]$} & {$[81.547]$} & {$[144.960]$} & {$[296.580]$} & {$[476.160]$} \\
\hline
\end{tabular}

Fuente: Estimación de los autores basada en la serie armonizada por Fundación ARU de encuestas de hogares (INE). 
Tabla 23: Efectos sobre la tasa de ahorro como proporción del ingreso, estimador Changes-inChanges

\begin{tabular}{|c|c|c|c|c|c|c|}
\hline & $C I C_{\text {promedio }}$ & $C I C_{q 0,1}$ & $C I C_{q 0,2}$ & $C I C_{q 0,5}$ & $C I C_{q 0,8}$ & $C I C_{q 0,9}$ \\
\hline \multicolumn{7}{|c|}{ Bolivia } \\
\hline \multirow[t]{2}{*}{$\tau^{C I C}$} & 1.214 & 1.951 & 0.448 & 0.125 & 0.006 & 0.040 \\
\hline & {$[0.610]^{* *}$} & {$[1.170]^{*}$} & {$[0.281]$} & {$[0.068]^{*}$} & {$[0.034]$} & [0.033] \\
\hline \multirow[t]{2}{*}{$\tau_{w / \operatorname{cov}}^{C I C}$} & 1.110 & 1.086 & 0.483 & -0.148 & -0.129 & -0.004 \\
\hline & {$[0.646]^{*}$} & {$[1.021]$} & {$[0.377]$} & {$[0.179]$} & {$[0.074]^{*}$} & {$[0.077]$} \\
\hline \multicolumn{7}{|c|}{ Mujeres } \\
\hline \multirow[t]{2}{*}{$\tau^{C I C}$} & 2.138 & 2.309 & 0.615 & 0.203 & 0.066 & 0.015 \\
\hline & {$[1.022]^{* *}$} & {$[2.218]$} & {$[0.468]$} & {$[0.134]$} & {$[0.063]$} & {$[0.056]$} \\
\hline \multirow[t]{2}{*}{$\tau_{w / \operatorname{cov}}^{C I C}$} & 2.243 & 3.209 & 0.557 & 0.156 & 0.041 & 0.169 \\
\hline & {$[1.163]^{*}$} & {$[2.437]$} & {$[0.816]$} & {$[0.316]$} & {$[0.143]$} & {$[0.127]$} \\
\hline \multicolumn{7}{|c|}{ Hombres } \\
\hline \multirow[t]{2}{*}{$\tau^{C I C}$} & 1.212 & 2.171 & 0.582 & 0.184 & 0.063 & 0.100 \\
\hline & {$[0.606]^{* *}$} & [2.183] & {$[0.448]$} & {$[0.096]^{*}$} & {$[0.055]$} & {$[0.050]^{* *}$} \\
\hline \multirow[t]{2}{*}{$\tau_{w / \operatorname{cov}}^{C I C}$} & 1.076 & 0.703 & 0.592 & -0.221 & -0.177 & -0.023 \\
\hline & {$[0.693]$} & [1.931] & {$[0.703]$} & {$[0.236]$} & {$[0.118]$} & {$[0.116]$} \\
\hline \multicolumn{7}{|c|}{ Mujer y hombre } \\
\hline$\tau^{C I C}$ & 0.294 & -0.138 & -0.265 & -0.238 & -0.179 & -0.085 \\
\hline \multirow{3}{*}{$\tau_{w / \operatorname{cov}}^{C I C}$} & {$[1.548]$} & {$[1.941]$} & {$[0.545]$} & {$[0.142]^{*}$} & {$[0.064]^{* * *}$} & [0.081] \\
\hline & 0.023 & -0.802 & 0.560 & -0.830 & -0.310 & -0.263 \\
\hline & [1.918] & {$[2.490]$} & {$[0.850]$} & {$[0.648]$} & {$[0.211]$} & {$[0.212]$} \\
\hline
\end{tabular}

Fuente: Estimación de los autores basada en la serie armonizada por Fundación ARU de encuestas de hogares (INE). 
Tabla 24: Efectos sobre la tasa de ahorro como proporción del consumo, estimador Changesin-Changes

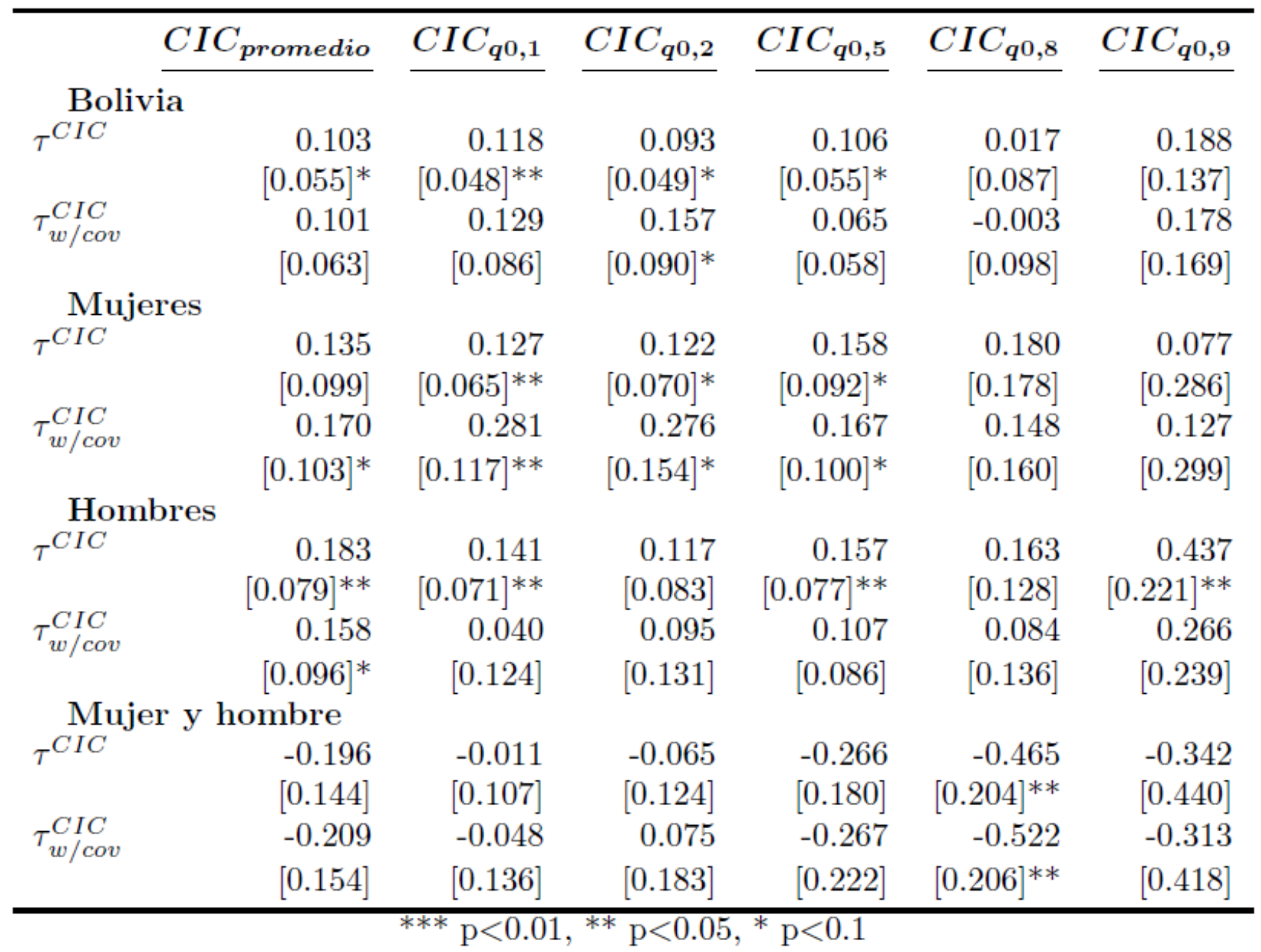

Fuente: Estimación de los autores basada en la serie armonizada por Fundación ARU de encuestas de hogares (INE). 
Tabla 25: Efectos sobre la tasa de ahorro aproximada $(\log y-\log c)$, estimador Changes-inChanges

\begin{tabular}{|c|c|c|c|c|c|c|}
\hline & $C I C_{\text {promedio }}$ & $C I C_{q 0,1}$ & $C I C_{q 0,2}$ & $C I C_{q 0,5}$ & $C I C_{q 0,8}$ & $C I C_{q 0,9}$ \\
\hline \multicolumn{7}{|c|}{ Bolivia } \\
\hline \multirow[t]{2}{*}{$\tau^{C I C}$} & 0.172 & 0.476 & 0.204 & 0.115 & 0.010 & 0.087 \\
\hline & {$[0.070]^{* *}$} & {$[0.211]^{* *}$} & {$[0.103]^{* *}$} & {$[0.058]^{* *}$} & {$[0.062]$} & {$[0.067]$} \\
\hline \multirow[t]{2}{*}{$\tau_{w / c o v}^{C I C}$} & 0.166 & 0.330 & 0.332 & 0.025 & -0.022 & 0.032 \\
\hline & {$[0.108]$} & {$[0.302]$} & {$[0.170]^{*}$} & {$[0.087]$} & {$[0.063]$} & {$[0.084]$} \\
\hline \multicolumn{7}{|c|}{ Mujeres } \\
\hline \multirow[t]{2}{*}{$\tau^{C I C}$} & 0.261 & 0.536 & 0.273 & 0.179 & 0.109 & 0.034 \\
\hline & {$[0.126]^{* *}$} & {$[0.343]$} & {$[0.161] *$} & {$[0.114]$} & {$[0.115]$} & [0.119] \\
\hline \multirow{2}{*}{$\tau_{w / c o v}^{C I C}$} & 0.321 & 0.796 & 0.385 & 0.144 & 0.109 & 0.137 \\
\hline & {$[0.134]^{* *}$} & {$[0.483]^{*}$} & {$[0.275]$} & {$[0.144]$} & {$[0.112]$} & {$[0.150]$} \\
\hline \multicolumn{7}{|c|}{ Hombres } \\
\hline \multirow[t]{2}{*}{$\tau^{C I C}$} & 0.235 & 0.547 & 0.260 & 0.170 & 0.102 & 0.209 \\
\hline & {$[0.116]^{* *}$} & {$[0.349]$} & {$[0.246]$} & {$[0.085]^{* *}$} & {$[0.094]$} & {$[0.103]^{* *}$} \\
\hline \multirow{2}{*}{$\tau_{w / c o v}^{C I C}$} & 0.198 & 0.389 & 0.226 & 0.128 & -0.026 & 0.036 \\
\hline & {$[0.116]^{*}$} & {$[0.403]$} & {$[0.290]$} & {$[0.105]$} & {$[0.078]$} & {$[0.101]$} \\
\hline \multicolumn{7}{|c|}{ Mujer y hombre } \\
\hline \multirow[t]{2}{*}{$\tau^{C I C}$} & -0.134 & -0.039 & -0.131 & -0.251 & -0.287 & -0.170 \\
\hline & {$[0.140]$} & {$[0.441]$} & {$[0.290]$} & {$[0.147]^{*}$} & {$[0.115]^{* *}$} & {$[0.178]$} \\
\hline \multirow[t]{2}{*}{$\tau_{w / \operatorname{cov}}^{C I C}$} & -0.178 & 0.141 & 0.158 & -0.205 & -0.442 & -0.255 \\
\hline & {$[0.199]$} & {$[0.467]$} & {$[0.331]$} & {$[0.220]$} & {$[0.154]^{* * *}$} & {$[0.167]$} \\
\hline
\end{tabular}

Fuente: Estimación de los autores basada en la serie armonizada por Fundación ARU de encuestas de hogares (INE). 
Tabla 26: Estimados de Diff-in-Diff de indicadores de nivel y sectores de participación

\begin{tabular}{|c|c|c|c|c|c|c|c|c|c|}
\hline & \multicolumn{3}{|c|}{ Bolivia } & \multicolumn{3}{|c|}{ Hombres } & \multicolumn{3}{|c|}{ Mujeres } \\
\hline & {$[55,60)$} & {$[60,65)$} & DID & {$[55,60)$} & {$[60,65)$} & DID & {$[55,60)$} & {$[60,65)$} & DID \\
\hline \multicolumn{10}{|c|}{ Participación } \\
\hline \multirow[t]{2}{*}{$\mathrm{T}=0$} & 0.80 & 0.74 & & 0.92 & 0.82 & & 0.69 & 0.66 & \\
\hline & {$[0.01]$} & {$[0.01]$} & -0.04 & {$[0.01]$} & {$[0.02]$} & 0.02 & {$[0.02]$} & {$[0.02]$} & -0.10 \\
\hline \multirow{2}{*}{$\mathrm{T}=1$} & 0.85 & 0.75 & {$[0.02]^{*}$} & 0.96 & 0.88 & {$[0.02]$} & 0.74 & 0.62 & {$[0.03]^{* * * *}$} \\
\hline & {$[0.01]$} & {$[0.01]$} & & {$[0.01]$} & {$[0.01]$} & & {$[0.01]$} & {$[0.02]$} & \\
\hline \multicolumn{10}{|c|}{ Trabajador familiar } \\
\hline \multirow[t]{2}{*}{$\mathrm{T}=0$} & 0.13 & 0.16 & & 0.02 & 0.03 & & 0.23 & 0.27 & \\
\hline & {$[0.01]$} & {$[0.01]$} & -0.01 & {$[0.01]$} & {$[0.01]$} & -0.01 & {$[0.02]$} & {$[0.02]$} & -0.02 \\
\hline \multirow[t]{2}{*}{$\mathrm{T}=1$} & 0.13 & 0.14 & {$[0.02]$} & 0.02 & 0.02 & {$[0.01]$} & 0.23 & 0.25 & {$[0.03]$} \\
\hline & {$[0.01]$} & {$[0.01]$} & & {$[0.00]$} & {$[0.00]$} & & {$[0.01]$} & {$[0.02]$} & \\
\hline \multicolumn{10}{|c|}{ Informal $\mathrm{n}$ /asalariado } \\
\hline \multirow[t]{2}{*}{$\mathrm{T}=0$} & 0.45 & 0.45 & & 0.57 & 0.62 & & 0.34 & 0.31 & \\
\hline & {$[0.01]$} & {$[0.01]$} & -0.02 & {$[0.02]$} & {$[0.02]$} & -0.02 & {$[0.02]$} & {$[0.02]$} & -0.03 \\
\hline \multirow[t]{2}{*}{$\mathrm{T}=1$} & 0.49 & 0.47 & {$[0.03]$} & 0.61 & 0.64 & {$[0.04]$} & 0.37 & 0.30 & {$[0.03]$} \\
\hline & {$[0.01]$} & {$[0.01]$} & & {$[0.02]$} & {$[0.02]$} & & {$[0.02]$} & {$[0.02]$} & \\
\hline \multicolumn{10}{|c|}{ Informal asalariado } \\
\hline \multirow[t]{2}{*}{$\mathrm{T}=0$} & 0.10 & 0.07 & & 0.17 & 0.10 & & 0.04 & 0.05 & \\
\hline & {$[0.01]$} & {$[0.01]$} & -0.01 & {$[0.01]$} & {$[0.01]$} & 0.02 & {$[0.01]$} & {$[0.01]$} & -0.03 \\
\hline \multirow[t]{2}{*}{$\mathrm{T}=1$} & 0.10 & 0.07 & {$[0.01]$} & 0.16 & 0.11 & {$[0.03]$} & 0.05 & 0.03 & {$[0.01]^{* * *}$} \\
\hline & {$[0.01]$} & {$[0.01]$} & & {$[0.01]$} & {$[0.01]$} & & {$[0.01]$} & {$[0.01]$} & \\
\hline \multicolumn{10}{|c|}{ Formal asalariado } \\
\hline \multirow[t]{2}{*}{$\mathrm{T}=0$} & 0.10 & 0.04 & & 0.15 & 0.07 & & 0.06 & 0.02 & \\
\hline & {$[0.01]$} & {$[0.01]$} & 0.00 & {$[0.01]$} & {$[0.01]$} & 0.02 & {$[0.01]$} & {$[0.01]$} & -0.02 \\
\hline \multirow[t]{2}{*}{$\mathrm{T}=1$} & 0.12 & 0.07 & {$[0.01]$} & 0.16 & 0.11 & {$[0.02]$} & 0.09 & 0.03 & {$[0.02]$} \\
\hline & {$[0.01]$} & {$[0.01]$} & & {$[0.01]$} & {$[0.01]$} & & {$[0.01]$} & {$[0.01]$} & \\
\hline
\end{tabular}

Fuente: Estimación de los autores basada en la serie armonizada por Fundación ARU de encuestas de hogares (INE).

Notas: Errores estándar entre corchetes (ver nota de figura 7). IAI=en el intervalo de edad (in age interval). 
Tabla 27: Efectos sobre la participación en el mercado laboral, estimador Changes-inChanges

\begin{tabular}{|c|c|c|c|}
\hline & $C I C_{\text {disc }}$ & $C I C_{d i s c} \mathrm{LB}$ & $C I C_{d i s c} \mathrm{UB}$ \\
\hline \multicolumn{4}{|c|}{ Bolivia } \\
\hline \multirow[t]{2}{*}{$\tau^{C I C}$} & -0.06 & -0.25 & 0.01 \\
\hline & {$[0.021]^{* * *}$} & {$[0.011]^{* * *}$} & {$[0.017]$} \\
\hline \multirow{2}{*}{$\tau_{w / c o v}^{C I C}$} & -0.07 & -0.07 & -0.07 \\
\hline & {$[0.028]^{* *}$} & {$[0.028]^{* *}$} & {$[0.028]^{* *}$} \\
\hline \multicolumn{4}{|c|}{ Mujeres } \\
\hline \multirow[t]{2}{*}{$\tau^{C I C}$} & -0.10 & -0.38 & -0.04 \\
\hline & {$[0.034]^{* * *}$} & {$[0.018]^{* * *}$} & {$[0.027]$} \\
\hline \multirow{2}{*}{$\tau_{w / \operatorname{cov}}^{C I C}$} & -0.12 & -0.12 & -0.12 \\
\hline & {$[0.039]^{* * *}$} & {$[0.039]^{* * *}$} & {$[0.039]^{* * *}$} \\
\hline \multicolumn{4}{|c|}{ Hombres } \\
\hline$\tau^{C I C}$ & -0.02 & -0.12 & 0.06 \\
\hline \multirow{3}{*}{$\tau_{w / \operatorname{cov}}^{C I C}$} & {$[0.023]$} & {$[0.011]^{* * *}$} & {$[0.019]^{* * *}$} \\
\hline & -0.01 & -0.01 & -0.01 \\
\hline & {$[0.033]$} & {$[0.033]$} & [0.033] \\
\hline
\end{tabular}

Fuente: Estimación de los autores basada en la serie armonizada por Fundación ARU de encuestas de hogares (INE). LB=límite inferior (lower bound), UB=límite superior (upper bound). 
Tabla 28: Efectos sobre sectores de inserción laboral, estimador Changes-in-Changes

(a) Trabajador familiar

\begin{tabular}{|c|c|c|c|}
\hline & $C I C_{d i s c}$ & $C I C_{d i s c} \mathrm{LB}$ & $C I C_{d i s c} \mathrm{UB}$ \\
\hline Bol & & & \\
\hline$\tau^{C I C}$ & -0.01 & -0.02 & 0.14 \\
\hline & {$[0.018]$} & {$[0.225]$} & {$[0.048]^{* * *}$} \\
\hline$\tau_{w \psi}^{C I C}$ & -0.02 & -0.02 & -0.02 \\
\hline & {$[0.026]$} & {$[0.026]$} & {$[0.026]$} \\
\hline $\mathrm{Mu}$ & eres & & \\
\hline$\tau^{C I C}$ & -0.02 & -0.02 & 0.25 \\
\hline & [0.031] & {$[0.203]$} & {$[0.086]^{* * * *}$} \\
\hline$\tau_{w / \text { cov }}^{C I C}$ & -0.03 & -0.04 & -0.03 \\
\hline & [0.040] & {$[0.040]$} & {$[0.040]$} \\
\hline Hol & bres & & \\
\hline$\tau^{C I C}$ & -0.00 & -0.01 & 0.02 \\
\hline & {$[0.010]$} & {$[0.254]$} & {$[0.012]$} \\
\hline$\tau_{w / c o v}^{C I C}$ & 0.01 & 0.01 & 0.01 \\
\hline & [0.015] & {$[0.015]$} & [0.015] \\
\hline
\end{tabular}

(c) Informal asalariado

\begin{tabular}{|c|c|c|c|}
\hline & $C I C_{d i s c}$ & $C I C_{d i s c} \mathrm{LB}$ & $C I C_{d i s c}$ UB \\
\hline \multicolumn{4}{|c|}{ Bolivia } \\
\hline \multirow[t]{2}{*}{$\tau^{C I C}$} & -0.01 & -0.93 & -0.00 \\
\hline & {$[0.014]$} & {$[0.244]^{* * *}$} & {$[0.025]$} \\
\hline \multirow{2}{*}{$\tau_{w / c o v}^{C I C}$} & -0.00 & -0.00 & -0.00 \\
\hline & {$[0.017]$} & {$[0.017]$} & {$[0.017]$} \\
\hline \multicolumn{4}{|c|}{ Mujeres } \\
\hline \multirow{2}{*}{$\tau^{C I C}$} & -0.03 & -0.97 & -0.02 \\
\hline & {$[0.014]^{* *}$} & {$[0.249]^{* * *}$} & {$[0.019]$} \\
\hline \multirow[t]{2}{*}{$\tau_{w / \operatorname{cov}}^{C I C}$} & -0.02 & -0.02 & -0.02 \\
\hline & {$[0.017]$} & {$[0.017]$} & {$[0.016]$} \\
\hline \multicolumn{4}{|c|}{ Hombres } \\
\hline \multirow[t]{2}{*}{$\tau^{C I C}$} & 0.02 & 0.01 & 0.11 \\
\hline & {$[0.021]$} & {$[0.242]$} & {$[0.036]^{* * *}$} \\
\hline \multirow[t]{2}{*}{$\tau_{w / c o v}^{C I C}$} & 0.01 & 0.01 & 0.01 \\
\hline & {$[0.025]$} & {$[0.025]$} & {$[0.025]$} \\
\hline
\end{tabular}

(b) Informal no asalariado

\begin{tabular}{|c|c|c|c|}
\hline & $C I C_{\text {disc }}$ & $C I C_{d i s c} \mathrm{LB}$ & $C I C_{d i s c}$ UB \\
\hline \multicolumn{4}{|c|}{ Bolivia } \\
\hline \multirow[t]{2}{*}{$\tau^{C I C}$} & -0.02 & -0.53 & 0.01 \\
\hline & {$[0.025]$} & {$[0.014]^{* * *}$} & {$[0.022]$} \\
\hline \multirow{2}{*}{$\tau_{w / c o v}^{C l C}$} & -0.02 & -0.02 & -0.02 \\
\hline & {$[0.030]$} & {$[0.030]$} & {$[0.030]$} \\
\hline \multicolumn{4}{|c|}{ Mujeres } \\
\hline \multirow[t]{2}{*}{$\tau^{C I C}$} & -0.03 & -0.70 & -0.01 \\
\hline & {$[0.035]$} & {$[0.188]^{* * *}$} & {$[0.095]$} \\
\hline \multirow{2}{*}{$\tau_{w / \text { cov }}^{C I C}$} & -0.06 & -0.06 & -0.06 \\
\hline & {$[0.040]$} & {$[0.040]$} & {$[0.040]$} \\
\hline \multicolumn{4}{|c|}{ Hombres } \\
\hline \multirow[t]{2}{*}{$\tau^{C I C}$} & -0.02 & -0.36 & 0.02 \\
\hline & {$[0.035]$} & {$[0.107]^{* * * *}$} & {$[0.171]$} \\
\hline \multirow{2}{*}{$\tau_{w / \text { cov }}^{C I C}$} & -0.01 & -0.01 & -0.01 \\
\hline & {$[0.037]$} & {$[0.037]$} & {$[0.037]$} \\
\hline
\end{tabular}

(d) Formal asalariado

\begin{tabular}{|c|c|c|c|}
\hline \multirow{4}{*}{$\underset{\tau^{C I C}}{\text { Boli }}$} & $C I C_{d i s c}$ & $C I C_{d i s c} \mathrm{LB}$ & $C I C_{d i s c}$ UB \\
\hline & & & \\
\hline & 0.00 & -0.93 & 0.02 \\
\hline & {$[0.014]$} & {$[0.246]^{* * *}$} & {$[0.015]$} \\
\hline \multirow{2}{*}{$\tau_{w / C o v}^{C I C}$} & 0.01 & 0.01 & 0.01 \\
\hline & {$[0.019]$} & {$[0.019]$} & {$[0.019]$} \\
\hline \multicolumn{4}{|c|}{ Mujeres } \\
\hline \multirow[t]{2}{*}{$\tau^{C I C}$} & -0.02 & -0.97 & 0.01 \\
\hline & {$[0.015]$} & {$[0.253]^{* * *}$} & [0.008] \\
\hline \multirow[t]{2}{*}{$\tau_{w / \text { cov }}^{C I C}$} & -0.00 & -0.00 & -0.00 \\
\hline & [0.022] & {$[0.022]$} & [0.021] \\
\hline \multicolumn{4}{|c|}{ Hombres } \\
\hline \multirow[t]{2}{*}{$\tau^{C I C}$} & 0.02 & -0.89 & 0.03 \\
\hline & {$[0.022]$} & {$[0.248]^{* * *}$} & {$[0.030]$} \\
\hline \multirow[t]{2}{*}{$\tau_{w / \text { cov }}^{C I C}$} & 0.02 & 0.02 & 0.02 \\
\hline & {$[0.031]$} & {$[0.031]$} & {$[0.030]$} \\
\hline
\end{tabular}

$$
* * * \mathrm{p}<0.01, * * \mathrm{p}<0.05, * \mathrm{p}<0.1
$$

Fuente: Estimación de los autores basada en la serie armonizada por Fundación ARU de encuestas de hogares (INE). $\mathrm{LB}=$ límite inferior (lower bound), $\mathrm{UB}=$ límite superior (upper bound). 
Tabla 29: Estimados de Diff-in-Diff de las horas trabajadas, salario por hora, e ingresos laborales mensuales

\begin{tabular}{|c|c|c|c|c|c|c|c|c|c|}
\hline & \multicolumn{3}{|c|}{ Bolivia } & \multicolumn{3}{|c|}{ Hombres } & \multicolumn{3}{|c|}{ Mujeres } \\
\hline & {$[55,60)$} & {$[60,65)$} & DID & {$[55,60)$} & {$[60,65)$} & DID & {$[55,60)$} & {$[60,65)$} & DID \\
\hline \multicolumn{10}{|c|}{ Intensidad de oferta total (log de horas por mes) } \\
\hline \multirow[t]{2}{*}{$\mathrm{T}=0$} & 2.78 & 2.50 & & 3.38 & 2.93 & & 2.25 & 2.11 & \\
\hline & {$[0.05]$} & {$[0.06]$} & -0.18 & {$[0.06]$} & {$[0.08]$} & 0.06 & {$[0.08]$} & {$[0.09]$} & -0.40 \\
\hline \multirow[t]{2}{*}{$\mathrm{T}=1$} & 3.01 & 2.56 & {$[0.10]^{*}$} & 3.58 & 3.19 & {$[0.12]$} & 2.47 & 1.93 & {$[0.16]^{* *}$} \\
\hline & {$[0.04]$} & {$[0.05]$} & & {$[0.04]$} & {$[0.06]$} & & {$[0.07]$} & {$[0.08]$} & \\
\hline \multicolumn{10}{|c|}{ Intensidad de oferta en la actividad primaria (log de horas por mes) } \\
\hline \multirow[t]{2}{*}{$\mathrm{T}=0$} & 2.74 & 2.46 & & 3.33 & 2.89 & & 2.22 & 2.07 & \\
\hline & {$[0.05]$} & {$[0.06]$} & -0.17 & {$[0.06]$} & {$[0.08]$} & 0.06 & {$[0.08]$} & {$[0.09]$} & -0.39 \\
\hline \multirow[t]{2}{*}{$\mathrm{T}=1$} & 2.97 & 2.52 & {$[0.10]^{*}$} & 3.52 & 3.15 & {$[0.12]$} & 2.44 & 1.90 & {$[0.16]^{* *}$} \\
\hline & {$[0.04]$} & {$[0.05]$} & & {$[0.04]$} & {$[0.06]$} & & {$[0.07]$} & {$[0.08]$} & \\
\hline \multicolumn{10}{|c|}{ Salario (log de Bs. 2012 por hora) } \\
\hline \multirow[t]{2}{*}{$\mathrm{T}=0$} & -1.23 & -1.97 & & 0.56 & -0.40 & & -2.79 & -3.40 & \\
\hline & {$[0.10]$} & {$[0.11]$} & -0.32 & {$[0.12]$} & {$[0.15]$} & 0.03 & {$[0.14]$} & {$[0.14]$} & -0.67 \\
\hline \multirow[t]{2}{*}{$\mathrm{T}=1$} & -0.48 & -1.54 & {$[0.20]$} & 1.26 & 0.34 & {$[0.23]$} & -2.12 & -3.39 & {$[0.27]^{* *}$} \\
\hline & {$[0.09]$} & {$[0.10]$} & & {$[0.08]$} & {$[0.11]$} & & {$[0.13]$} & {$[0.13]$} & \\
\hline \multicolumn{10}{|c|}{ Ingreso laboral mensual (log de Bs. 2012 por mes) } \\
\hline \multirow[t]{2}{*}{$\mathrm{T}=0$} & 3.67 & 2.73 & & 5.76 & 4.60 & & 1.83 & 1.05 & \\
\hline & {$[0.12]$} & {$[0.13]$} & -0.32 & {$[0.13]$} & {$[0.17]$} & 0.12 & {$[0.16]$} & {$[0.17]$} & -0.75 \\
\hline \multirow[t]{2}{*}{$\mathrm{T}=1$} & 4.44 & 3.19 & {$[0.23]$} & 6.49 & 5.45 & {$[0.26]$} & 2.51 & 0.97 & {$[0.31]^{* *}$} \\
\hline & {$[0.10]$} & {$[0.11]$} & & {$[0.09]$} & {$[0.13]$} & & {$[0.15]$} & {$[0.15]$} & \\
\hline
\end{tabular}

Fuente: Estimación de los autores basada en la serie armonizada por Fundación ARU de encuestas de hogares (INE).

Notas: Errores estándar entre corchetes (ver nota de figura 7). IAI=en el intervalo de edad (in age interval). 
Tabla 30: Efectos sobre las (log) horas trabajadas en total, estimador Changes-in-Changes

\begin{tabular}{|c|c|c|c|c|c|c|}
\hline & $C I C_{\text {promedio }}$ & $C I C_{q 0,1}$ & $C I C_{q 0,2}$ & $C I C_{q 0,5}$ & $C I C_{q 0,8}$ & $C I C_{q 0,9}$ \\
\hline \multicolumn{7}{|c|}{ Bolivia } \\
\hline \multirow[t]{2}{*}{$\tau^{C I C}$} & -0.226 & 0.000 & 0.000 & 0.000 & 0.000 & 0.000 \\
\hline & {$[0.101]^{* *}$} & {$[0.000]$} & {$[0.811]$} & {$[0.054]$} & {$[0.035]$} & {$[0.022]$} \\
\hline \multirow{2}{*}{$\tau_{w / \operatorname{cov}}^{C I C}$} & -0.245 & -0.268 & -1.462 & -0.094 & 0.096 & -0.101 \\
\hline & {$[0.134]^{*}$} & {$[0.160]^{*}$} & {$[0.668]^{* *}$} & [0.098] & {$[0.105]$} & {$[0.075]$} \\
\hline \multicolumn{7}{|c|}{ Mujeres } \\
\hline \multirow[t]{2}{*}{$\tau^{C I C}$} & -0.416 & 0.000 & 0.000 & -0.357 & -0.036 & 0.105 \\
\hline & {$[0.147]^{* * *}$} & {$[0.000]$} & {$[0.000]$} & {$[0.136]^{* * *}$} & {$[0.059]$} & {$[0.065]$} \\
\hline \multirow{2}{*}{$\tau_{w / c o v}^{C I C}$} & -0.495 & -0.102 & -0.267 & -0.298 & -0.179 & 0.014 \\
\hline & {$[0.177]^{* * *}$} & {$[0.089]$} & {$[0.172]$} & {$[0.184]$} & {$[0.109]^{*}$} & {$[0.071]$} \\
\hline \multicolumn{7}{|c|}{ Hombres } \\
\hline \multirow[t]{2}{*}{$\tau^{C I C}$} & -0.087 & 0.000 & 0.000 & 0.000 & 0.000 & -0.028 \\
\hline & {$[0.123]$} & {$[0.868]$} & {$[0.223]$} & {$[0.033]$} & {$[0.057]$} & {$[0.035]$} \\
\hline \multirow[t]{2}{*}{$\tau_{w / c o v}^{C I C}$} & 0.016 & 0.183 & 0.062 & 0.156 & -0.105 & 0.000 \\
\hline & {$[0.156]$} & {$[0.935]$} & {$[0.316]$} & {$[0.107]$} & {$[0.069]$} & {$[0.052]$} \\
\hline
\end{tabular}

Fuente: Estimación de los autores basada en la serie armonizada por Fundación ARU de encuestas de hogares (INE). 
Tabla 31: Efectos sobre las (log) horas trabajadas en la actividad primaria, estimador Changes-in-Changes

\begin{tabular}{|c|c|c|c|c|c|c|}
\hline & $C I C_{\text {promedio }}$ & $C I C_{q 0,1}$ & $C I C_{q 0,2}$ & $C I C_{q 0,5}$ & $C I C_{q 0,8}$ & $C I C_{q 0,9}$ \\
\hline \multicolumn{7}{|c|}{ Bolivia } \\
\hline \multirow[t]{2}{*}{$\tau^{C I C}$} & -0.224 & 0.000 & 0.000 & -0.054 & 0.000 & 0.000 \\
\hline & {$[0.095]^{* *}$} & {$[0.000]$} & {$[0.764]$} & {$[0.061]$} & {$[0.018]$} & {$[0.066]$} \\
\hline \multirow{2}{*}{$\tau_{w / c o v}^{C I C}$} & -0.245 & -0.221 & -1.570 & -0.090 & 0.069 & -0.065 \\
\hline & {$[0.131]^{*}$} & [0.155] & {$[0.654]^{* *}$} & {$[0.117]$} & {$[0.100]$} & {$[0.084]$} \\
\hline \multicolumn{7}{|c|}{ Mujeres } \\
\hline \multirow[t]{2}{*}{$\tau^{C I C}$} & -0.418 & 0.000 & 0.000 & -0.405 & -0.134 & 0.000 \\
\hline & {$[0.142]^{* * *}$} & {$[0.000]$} & {$[0.000]$} & {$[0.144]^{* * *}$} & {$[0.050]^{* * *}$} & {$[0.066]$} \\
\hline \multirow{2}{*}{$\tau_{w / c o v}^{C I C}$} & -0.488 & -0.067 & -0.257 & -0.267 & -0.212 & -0.028 \\
\hline & {$[0.169]^{* * *}$} & [0.088] & {$[0.157]$} & {$[0.189]$} & {$[0.111]^{*}$} & {$[0.085]$} \\
\hline \\
\hline \multicolumn{7}{|c|}{$\begin{array}{l}\text { Hombres } \\
\tau^{C I C}\end{array}$} \\
\hline \multirow{3}{*}{$\tau_{w / \operatorname{cov}}^{C I C}$} & {$[0.120]$} & [0.811] & {$[0.246]$} & {$[0.068]$} & {$[0.038]^{*}$} & [0.046] \\
\hline & 0.027 & 0.189 & 0.102 & 0.119 & -0.036 & -0.059 \\
\hline & {$[0.149]$} & {$[0.897]$} & {$[0.399]$} & {$[0.102]$} & {$[0.076]$} & {$[0.066]$} \\
\hline
\end{tabular}

Fuente: Estimación de los autores basada en la serie armonizada por Fundación ARU de encuestas de hogares (INE). 
Tabla 32: Efectos sobre el (log) salario por hora, estimador Changes-in-Changes

\begin{tabular}{|c|c|c|c|c|c|c|}
\hline & $C I C_{\text {promedio }}$ & $C I C_{q 0,1}$ & $C I C_{q 0,2}$ & $C I C_{q 0,5}$ & $C I C_{q 0,8}$ & $C I C_{q 0,9}$ \\
\hline \multicolumn{7}{|c|}{ Bolivia } \\
\hline \multirow[t]{2}{*}{$\tau^{C I C}$} & -0.426 & 0.000 & 0.000 & -0.590 & -0.090 & -0.153 \\
\hline & {$[0.209]^{* *}$} & {$[0.000]$} & {$[0.000]$} & {$[0.280]^{* *}$} & {$[0.109]$} & [0.101] \\
\hline \multirow{2}{*}{$\tau_{w / c o v}^{C I C}$} & -0.437 & 0.119 & 0.045 & -0.742 & 0.074 & 0.242 \\
\hline & {$[0.270]$} & {$[0.100]$} & {$[0.136]$} & {$[0.391]^{*}$} & [0.199] & {$[0.179]$} \\
\hline \multicolumn{7}{|c|}{ Mujeres } \\
\hline \multirow[t]{2}{*}{$\tau^{C I C}$} & -0.714 & 0.000 & 0.000 & 0.000 & -0.554 & -0.310 \\
\hline & {$[0.281]^{* *}$} & {$[0.000]$} & {$[0.000]$} & [1.061] & {$[0.202]^{* * *}$} & {$[0.222]$} \\
\hline \multirow{2}{*}{$\tau_{w / \operatorname{cov}}^{C I C}$} & -0.689 & 0.020 & 0.137 & -0.166 & -0.828 & -0.439 \\
\hline & {$[0.315]^{* *}$} & {$[0.073]$} & {$[0.097]$} & [1.099] & {$[0.304]^{* * *}$} & {$[0.264]^{*}$} \\
\hline \multicolumn{7}{|c|}{ Hombres } \\
\hline \multirow[t]{2}{*}{$\tau^{C I C}$} & -0.220 & 0.000 & -0.576 & 0.015 & 0.024 & 0.053 \\
\hline & {$[0.224]$} & [0.948] & {$[0.677]$} & {$[0.143]$} & [0.128] & [0.133] \\
\hline \multirow[t]{2}{*}{$\tau_{w / \operatorname{cov}}^{C I C}$} & -0.367 & -2.807 & -0.916 & -0.175 & 0.203 & 0.034 \\
\hline & {$[0.246]$} & {$[1.080]^{* * *}$} & {$[0.774]$} & {$[0.204]$} & {$[0.158]$} & {$[0.203]$} \\
\hline
\end{tabular}

Fuente: Estimación de los autores basada en la serie armonizada por Fundación ARU de encuestas de hogares (INE). 
Tabla 33: Efectos sobre el (log) ingreso laboral mensual, estimador Changes-in-Changes

\begin{tabular}{|c|c|c|c|c|c|c|}
\hline & CI $C_{\text {promedio }}$ & $C I C_{q 0,1}$ & $C I C_{q 0,2}$ & $C I C_{q 0,5}$ & $C I C_{q 0,8}$ & $C I C_{q 0,9}$ \\
\hline \multicolumn{7}{|c|}{ Bolivia } \\
\hline \multirow{2}{*}{$\tau^{C I C}$} & -0.454 & 0.000 & 0.000 & -0.472 & 0.026 & -0.068 \\
\hline & {$[0.234]^{*}$} & {$[0.000]$} & {$[0.000]$} & {$[0.253]^{*}$} & {$[0.099]$} & {$[0.082]$} \\
\hline \multirow[t]{2}{*}{$\tau_{w / c o v}^{C I C}$} & -0.461 & 0.202 & 0.015 & -0.668 & 0.020 & 0.116 \\
\hline & {$[0.330]$} & {$[0.090]^{* *}$} & {$[0.154]$} & {$[0.464]$} & {$[0.248]$} & {$[0.174]$} \\
\hline \multicolumn{7}{|c|}{ Mujeres } \\
\hline \multirow[t]{2}{*}{$\tau^{C I C}$} & -0.812 & 0.000 & 0.000 & 0.000 & -0.614 & -0.265 \\
\hline & {$[0.320]^{* *}$} & {$[0.000]$} & {$[0.000]$} & [1.459] & {$[0.238]^{* * *}$} & {$[0.154]^{*}$} \\
\hline \multirow{2}{*}{$\tau_{w / \operatorname{cov}}^{C I C}$} & -0.742 & 0.056 & 0.190 & -0.266 & -0.985 & -0.486 \\
\hline & {$[0.327]^{* *}$} & {$[0.125]$} & {$[0.086]^{* *}$} & {$[1.256]$} & {$[0.265]^{* * *}$} & {$[0.198]^{* *}$} \\
\hline \multicolumn{7}{|c|}{ Hombres } \\
\hline \multirow[t]{2}{*}{$\tau^{C I C}$} & -0.216 & 0.000 & -0.473 & -0.033 & -0.076 & 0.092 \\
\hline & {$[0.258]$} & {$[1.349]$} & {$[0.641]$} & {$[0.175]$} & {$[0.112]$} & [0.138] \\
\hline \multirow{2}{*}{$\tau_{w / \operatorname{cov}}^{C I C}$} & -0.394 & -4.143 & -0.825 & -0.116 & 0.175 & 0.164 \\
\hline & {$[0.277]$} & {$[1.417]^{* * *}$} & {$[0.602]$} & {$[0.183]$} & {$[0.162]$} & {$[0.151]$} \\
\hline
\end{tabular}

Fuente: Estimación de los autores basada en la serie armonizada por Fundación ARU de encuestas de hogares (INE). 
Tabla 34: Estimados de Diff-in-Diff de indicadores de nivel y sector de participación de futuros beneficiarios

\begin{tabular}{|c|c|c|c|c|c|c|c|c|c|}
\hline & \multicolumn{3}{|c|}{ Bolivia } & \multicolumn{3}{|c|}{ Hombres } & \multicolumn{3}{|c|}{ Mujeres } \\
\hline & {$[50,54)$} & {$[55,60)$} & DID & {$[50,54)$} & {$[55,60)$} & DID & {$[50,54)$} & {$[55,60)$} & DID \\
\hline \multicolumn{10}{|c|}{ Participación } \\
\hline \multirow[t]{2}{*}{$\mathrm{T}=0$} & 0.85 & 0.81 & & 0.97 & 0.92 & & 0.75 & 0.70 & \\
\hline & {$[0.01]$} & {$[0.01]$} & 0.03 & {$[0.01]$} & {$[0.01]$} & 0.02 & {$[0.02]$} & {$[0.02]$} & 0.03 \\
\hline \multirow[t]{2}{*}{$\mathrm{T}=1$} & 0.88 & 0.87 & {$[0.02]^{*}$} & 0.98 & 0.96 & {$[0.02]$} & 0.78 & 0.76 & {$[0.03]$} \\
\hline & {$[0.01]$} & {$[0.01]$} & & {$[0.00]$} & {$[0.01]$} & & {$[0.01]$} & {$[0.02]$} & \\
\hline \multicolumn{10}{|c|}{ Trabajador familiar } \\
\hline \multirow[t]{2}{*}{$\mathrm{T}=0$} & 0.11 & 0.13 & & 0.01 & 0.02 & & 0.21 & 0.23 & \\
\hline & {$[0.01]$} & {$[0.01]$} & -0.04 & {$[0.00]$} & {$[0.01]$} & -0.00 & {$[0.01]$} & {$[0.02]$} & -0.06 \\
\hline \multirow[t]{2}{*}{$\mathrm{T}=1$} & 0.11 & 0.08 & {$[0.02]^{* *}$} & 0.01 & 0.01 & {$[0.01]$} & 0.20 & 0.17 & {$[0.03]^{* *}$} \\
\hline & {$[0.01]$} & {$[0.01]$} & & {$[0.00]$} & {$[0.00]$} & & {$[0.01]$} & {$[0.01]$} & \\
\hline \multicolumn{10}{|c|}{ Informal $\mathrm{n}$ /asalariado } \\
\hline \multirow{2}{*}{$\mathrm{T}=0$} & 0.46 & 0.46 & & 0.59 & 0.57 & & 0.34 & 0.35 & \\
\hline & {$[0.01]$} & {$[0.01]$} & 0.06 & {$[0.02]$} & {$[0.02]$} & 0.06 & {$[0.02]$} & {$[0.02]$} & 0.05 \\
\hline \multirow[t]{2}{*}{$\mathrm{T}=1$} & 0.47 & 0.53 & {$[0.03]^{* *}$} & 0.57 & 0.62 & {$[0.04]^{*}$} & 0.37 & 0.43 & {$[0.04]$} \\
\hline & {$[0.01]$} & {$[0.01]$} & & {$[0.02]$} & {$[0.02]$} & & {$[0.02]$} & {$[0.02]$} & \\
\hline \multicolumn{10}{|c|}{ Informal asalariado } \\
\hline \multirow[t]{2}{*}{$\mathrm{T}=0$} & 0.11 & 0.11 & & 0.16 & 0.18 & & 0.07 & 0.05 & \\
\hline & {$[0.01]$} & {$[0.01]$} & -0.01 & {$[0.01]$} & {$[0.02]$} & -0.04 & {$[0.01]$} & {$[0.01]$} & 0.00 \\
\hline \multirow[t]{2}{*}{$\mathrm{T}=1$} & 0.13 & 0.12 & {$[0.02]$} & 0.18 & 0.16 & {$[0.03]$} & 0.08 & 0.06 & {$[0.02]$} \\
\hline & {$[0.01]$} & {$[0.01]$} & & {$[0.01]$} & {$[0.01]$} & & {$[0.01]$} & {$[0.01]$} & \\
\hline \multicolumn{10}{|c|}{ Formal asalariado } \\
\hline \multirow[t]{2}{*}{$\mathrm{T}=0$} & 0.15 & 0.10 & & 0.19 & 0.14 & & 0.11 & 0.06 & \\
\hline & {$[0.01]$} & {$[0.01]$} & 0.02 & {$[0.01]$} & {$[0.01]$} & -0.00 & {$[0.01]$} & {$[0.01]$} & 0.04 \\
\hline \multirow[t]{2}{*}{$\mathrm{T}=1$} & 0.15 & 0.13 & {$[0.02]$} & 0.21 & 0.16 & {$[0.03]$} & 0.10 & 0.09 & {$[0.02]^{*}$} \\
\hline & {$[0.01]$} & {$[0.01]$} & & {$[0.01]$} & {$[0.01]$} & & {$[0.01]$} & {$[0.01]$} & \\
\hline
\end{tabular}

Fuente: Estimación de los autores basada en la serie armonizada por Fundación ARU de encuestas de hogares (INE).

Notas: Errores estándar entre corchetes (ver nota de figura 7). IAI=en el intervalo de edad (in age interval). 
Tabla 35: Efectos sobre la partipación en el mercado laboral de futuros beneficiarios, estimador Changes-in-Changes

\begin{tabular}{|c|c|c|c|}
\hline & $C I C_{d i s c}$ & $C I C_{d i s c} \mathrm{LB}$ & $C I C_{d i s c} \mathrm{UB}$ \\
\hline \multicolumn{4}{|c|}{ Bolivia } \\
\hline \multirow[t]{2}{*}{$\tau^{C I C}$} & 0.03 & -0.13 & 0.06 \\
\hline & {$[0.020]$} & {$[0.009]^{* * *}$} & {$[0.019]^{* * *}$} \\
\hline \multirow{2}{*}{$\tau_{w / \operatorname{cov}}^{C I C}$} & 0.02 & 0.02 & 0.02 \\
\hline & {$[0.024]$} & {$[0.024]$} & {$[0.024]$} \\
\hline \multicolumn{4}{|c|}{ Mujeres } \\
\hline \multirow[t]{2}{*}{$\tau^{C I C}$} & 0.03 & -0.24 & 0.06 \\
\hline & {$[0.030]$} & {$[0.087]^{* * *}$} & [0.193] \\
\hline \multirow{2}{*}{$\tau_{w / c o v}^{C I C}$} & 0.01 & 0.01 & 0.01 \\
\hline & {$[0.036]$} & {$[0.036]$} & {$[0.036]$} \\
\hline \multicolumn{4}{|c|}{ Hombres } \\
\hline$\tau^{C I C}$ & 0.00 & -0.04 & 0.04 \\
\hline \multirow{3}{*}{$\tau_{w / \operatorname{cov}}^{C I C}$} & {$[0.019]$} & {$[0.026]$} & {$[0.243]$} \\
\hline & 0.01 & 0.01 & 0.01 \\
\hline & {$[0.020]$} & {$[0.020]$} & {$[0.020]$} \\
\hline
\end{tabular}

Fuente: Estimación de los autores basada en la serie armonizada por Fundación ARU de encuestas de hogares (INE). $\mathrm{LB}=$ límite inferior (lower bound), UB=límite superior (upper bound). 
Tabla 36: Efectos sobre sectores de participación laboral de futuros beneficiarios, estimador Changes-in-Changes

\section{(a) Trabajador familiar}

\begin{tabular}{|c|c|c|c|}
\hline & $C I C_{d i s c}$ & $C I C_{d i s c} \mathrm{LB}$ & $C I C_{d i s c} \mathrm{UB}$ \\
\hline \multicolumn{4}{|c|}{ Bolivia } \\
\hline \multirow{2}{*}{$\tau^{C I C}$} & -0.03 & -0.04 & 0.08 \\
\hline & {$[0.015]^{* *}$} & {$[0.230]$} & {$[0.040]^{* *}$} \\
\hline \multirow[t]{2}{*}{$\tau_{w / c o v}^{C I C}$} & -0.04 & -0.04 & -0.04 \\
\hline & {$[0.022]^{*}$} & {$[0.022]^{*}$} & {$[0.022]^{*}$} \\
\hline \multicolumn{4}{|c|}{ Mujeres } \\
\hline \multirow[t]{2}{*}{$\tau^{C I C}$} & -0.06 & -0.07 & 0.17 \\
\hline & {$[0.030]^{* *}$} & {$[0.209]$} & {$[0.075]^{* *}$} \\
\hline \multirow[t]{2}{*}{$\tau_{w / C o v}^{C I C}$} & -0.06 & -0.06 & -0.06 \\
\hline & {$[0.030]^{* *}$} & {$[0.030]^{* *}$} & {$[0.029]^{* *}$} \\
\hline \multicolumn{4}{|c|}{ Hombres } \\
\hline \multirow[t]{2}{*}{$\tau^{C I C}$} & 0.00 & -0.00 & 0.01 \\
\hline & {$[0.006]$} & {$[0.255]$} & {$[0.008]^{*}$} \\
\hline \multirow[t]{2}{*}{$\tau_{w / c o v}^{C I C}$} & 0.00 & 0.00 & 0.00 \\
\hline & {$[0.014]$} & {$[0.014]$} & {$[0.014]$} \\
\hline
\end{tabular}

\section{(c) Informal asalariado}

\begin{tabular}{|c|c|c|c|}
\hline & $C I C_{d i s c}$ & $C I C_{d i s c} \mathrm{LB}$ & $C I C_{d i s c} \mathrm{UB}$ \\
\hline \multicolumn{4}{|c|}{ Bolivia } \\
\hline \multirow[t]{2}{*}{$\tau^{C I C}$} & -0.01 & -0.88 & 0.01 \\
\hline & {$[0.016]$} & {$[0.230]^{* * *}$} & {$[0.034]$} \\
\hline \multirow{2}{*}{$\tau_{w / \operatorname{cov}}^{C I C}$} & -0.01 & -0.01 & -0.01 \\
\hline & {$[0.020]$} & {$[0.020]$} & {$[0.020]$} \\
\hline \multicolumn{4}{|c|}{ Mujeres } \\
\hline \multirow[t]{2}{*}{$\tau^{C I C}$} & 0.00 & -0.94 & 0.02 \\
\hline & {$[0.019]$} & {$[0.250]^{* * *}$} & {$[0.020]$} \\
\hline \multirow{2}{*}{$\tau_{w / \text { cov }}^{C I C}$} & 0.01 & 0.01 & 0.01 \\
\hline & {$[0.019]$} & {$[0.019]$} & {$[0.019]$} \\
\hline \multicolumn{4}{|c|}{ Hombres } \\
\hline \multirow[t]{2}{*}{$\tau^{C I C}$} & -0.03 & -0.84 & -0.01 \\
\hline & {$[0.026]$} & {$[0.216]^{* * *}$} & {$[0.053]$} \\
\hline \multirow{2}{*}{$\tau_{w / \text { cov }}^{C I C}$} & -0.05 & -0.05 & -0.05 \\
\hline & {$[0.029]^{*}$} & {$[0.029]^{*}$} & {$[0.029]^{*}$} \\
\hline
\end{tabular}

(b) Informal no asalariado

\begin{tabular}{|c|c|c|c|}
\hline & $C I C_{d i s c}$ & $C I C_{d i s c} \mathrm{LB}$ & $C I C_{d i s c} \mathrm{UB}$ \\
\hline \multicolumn{4}{|c|}{ Bolivia } \\
\hline \multirow[t]{2}{*}{$\tau^{C I C}$} & 0.06 & -0.47 & 0.07 \\
\hline & {$[0.027]^{* *}$} & {$[0.152]^{* * *}$} & [0.132] \\
\hline \multirow[t]{2}{*}{$\tau_{w / \text { cov }}^{C I C}$} & 0.06 & 0.06 & 0.06 \\
\hline & {$[0.037]^{*}$} & {$[0.037]^{*}$} & {$[0.037]^{*}$} \\
\hline \multicolumn{4}{|c|}{ Mujeres } \\
\hline \multirow[t]{2}{*}{$\tau^{C I C}$} & 0.05 & -0.57 & 0.08 \\
\hline & {$[0.036]$} & {$[0.184]^{* * *}$} & [0.111] \\
\hline \multirow[t]{2}{*}{$\tau_{w / c o v}^{C I C}$} & 0.03 & 0.03 & 0.03 \\
\hline & {$[0.047]$} & {$[0.047]$} & {$[0.047]$} \\
\hline \multicolumn{4}{|c|}{ Hombres } \\
\hline \multirow[t]{2}{*}{$\tau^{C I C}$} & 0.06 & 0.05 & 0.62 \\
\hline & {$[0.035]^{*}$} & [0.128] & {$[0.168]^{* *}$} \\
\hline \multirow[t]{2}{*}{$\tau_{w / c o v}^{C I C}$} & 0.06 & 0.06 & 0.06 \\
\hline & {$[0.038]$} & {$[0.038]$} & {$[0.038$} \\
\hline
\end{tabular}

\section{(d) Formal asalariado}

\begin{tabular}{|c|c|c|c|}
\hline & $C I C_{\text {disc }}$ & $C I C_{d i s c} \mathrm{LB}$ & $C I C_{d i s c} \mathrm{UB}$ \\
\hline \multicolumn{4}{|c|}{ Bolivia } \\
\hline \multirow[t]{2}{*}{$\tau^{C I C}$} & 0.02 & -0.87 & 0.03 \\
\hline & {$[0.016]$} & {$[0.238]^{* * *}$} & {$[0.035]$} \\
\hline \multirow[t]{2}{*}{$\tau_{w / c o v}^{C I C}$} & 0.02 & 0.02 & 0.02 \\
\hline & {$[0.023]$} & {$[0.023]$} & {$[0.023]$} \\
\hline \multicolumn{4}{|c|}{ Mujeres } \\
\hline \multirow[t]{2}{*}{$\tau^{C I C}$} & 0.03 & 0.03 & 0.09 \\
\hline & {$[0.016]^{* *}$} & {$[0.249]$} & {$[0.024] * * *$} \\
\hline \multirow[t]{2}{*}{$\tau_{w / c o v}^{C I C}$} & 0.03 & 0.03 & 0.03 \\
\hline & [0.026] & {$[0.026]$} & {$[0.026]$} \\
\hline \multicolumn{4}{|c|}{ Hombres } \\
\hline \multirow[t]{2}{*}{$\tau^{C I C}$} & -0.00 & -0.84 & 0.02 \\
\hline & {$[0.034]$} & {$[0.231]^{* * *}$} & {$[0.052]$} \\
\hline \multirow{2}{*}{$\tau_{w / c o v}^{C I C}$} & 0.00 & 0.00 & 0.00 \\
\hline & {$[0.031]$} & {$[0.031]$} & [0.031] \\
\hline
\end{tabular}

$$
* * * \mathrm{p}<0.01, * * \mathrm{p}<0.05, * \mathrm{p}<0.1
$$

Fuente: Estimación de los autores basada en la serie armonizada por Fundación ARU de encuestas de hogares (INE). LB=límite inferior (lower bound), UB=límite superior (upper bound). 
Tabla 37: Estimados de Diff-in-Diff de las horas trabajadas, salario por hora, e ingresos laborales mensuales de futuros beneficiarios

\begin{tabular}{|c|c|c|c|c|c|c|c|c|c|}
\hline & \multicolumn{3}{|c|}{ Bolivia } & \multicolumn{3}{|c|}{ Hombres } & \multicolumn{3}{|c|}{ Mujeres } \\
\hline & {$[50,54)$} & {$[55,60)$} & DID & {$[50,54)$} & {$[55,60)$} & DID & {$[50,54)$} & {$[55,60)$} & DID \\
\hline \multicolumn{10}{|c|}{ Intensidad de oferta total (log de horas por mes) } \\
\hline \multirow[t]{2}{*}{$\mathrm{T}=0$} & 3.00 & 2.82 & & 3.62 & 3.38 & & 2.41 & 2.27 & \\
\hline & {$[0.04]$} & {$[0.05]$} & 0.15 & {$[0.04]$} & {$[0.06]$} & 0.18 & {$[0.07]$} & {$[0.08]$} & 0.05 \\
\hline \multirow[t]{2}{*}{$\mathrm{T}=1$} & 3.15 & 3.12 & {$[0.09]$} & 3.68 & 3.61 & {$[0.09]^{* *}$} & 2.62 & 2.53 & {$[0.15]$} \\
\hline & {$[0.04]$} & {$[0.04]$} & & {$[0.03]$} & {$[0.04]$} & & {$[0.07]$} & {$[0.08]$} & \\
\hline \multicolumn{10}{|c|}{ Intensidad de oferta en la actividad primaria (log de horas por mes) } \\
\hline \multirow[t]{2}{*}{$\mathrm{T}=0$} & 2.96 & 2.77 & & 3.58 & 3.32 & & 2.37 & 2.23 & \\
\hline & {$[0.04]$} & {$[0.05]$} & 0.16 & {$[0.04]$} & {$[0.06]$} & 0.19 & {$[0.07]$} & {$[0.08]$} & 0.05 \\
\hline \multirow[t]{2}{*}{$\mathrm{T}=1$} & 3.10 & 3.07 & {$[0.09]^{*}$} & 3.62 & 3.56 & {$[0.09]^{* *}$} & 2.57 & 2.49 & {$[0.15]$} \\
\hline & {$[0.04]$} & {$[0.04]$} & & {$[0.03]$} & {$[0.04]$} & & {$[0.07]$} & {$[0.08]$} & \\
\hline \multicolumn{10}{|c|}{ Salario (log de Bs. 2012 por hora) } \\
\hline \multirow[t]{2}{*}{$\mathrm{T}=0$} & -0.47 & -1.06 & & 1.23 & 0.63 & & -2.08 & -2.73 & \\
\hline & {$[0.09]$} & {$[0.11]$} & 0.64 & {$[0.09]$} & {$[0.12]$} & 0.37 & {$[0.14]$} & {$[0.15]$} & 0.71 \\
\hline \multirow[t]{2}{*}{$\mathrm{T}=1$} & -0.03 & 0.01 & {$[0.19]^{* * * *}$} & 1.58 & 1.35 & {$[0.19]^{* * *}$} & -1.67 & -1.60 & {$[0.29]^{* * *}$} \\
\hline & {$[0.08]$} & {$[0.09]$} & & {$[0.07]$} & {$[0.08]$} & & {$[0.13]$} & {$[0.16]$} & \\
\hline \multicolumn{10}{|c|}{ Ingreso laboral mensual (log de Bs. 2012 por mes) } \\
\hline \multirow[t]{2}{*}{$\mathrm{T}=0$} & 4.47 & 3.86 & & 6.49 & 5.85 & & 2.56 & 1.90 & \\
\hline & {$[0.11]$} & {$[0.12]$} & 0.61 & {$[0.10]$} & {$[0.13]$} & 0.21 & {$[0.16]$} & {$[0.17]$} & 0.78 \\
\hline \multirow[t]{2}{*}{$\mathrm{T}=1$} & 5.02 & 5.02 & {$[0.22]^{* * *}$} & 7.01 & 6.58 & {$[0.20]$} & 3.01 & 3.12 & {$[0.33]^{* * *}$} \\
\hline & {$[0.10]$} & {$[0.10]$} & & {$[0.07]$} & {$[0.09]$} & & {$[0.15]$} & {$[0.18]$} & \\
\hline
\end{tabular}

Fuente: Estimación de los autores basada en la serie armonizada por Fundación ARU de encuestas de hogares (INE).

Notas: Errores estándar entre corchetes (ver nota de figura 7). IAI=en el intervalo de edad (in age interval). 
Tabla 38: Efectos sobre las $(\log )$ horas trabajadas en total de futuros beneficiarios, estimador Changes-in-Changes

\begin{tabular}{|c|c|c|c|c|c|c|}
\hline & $C I C_{\text {promedio }}$ & $C I C_{q 0,1}$ & $C I C_{q 0,2}$ & $C I C_{q 0,5}$ & $C I C_{q 0,8}$ & $C I C_{q 0,9}$ \\
\hline \multicolumn{7}{|c|}{ Bolivia } \\
\hline \multirow[t]{2}{*}{$\tau^{C I C}$} & 0.131 & 0.000 & 0.405 & 0.000 & -0.049 & -0.028 \\
\hline & {$[0.103]$} & {$[0.000]$} & {$[0.971]$} & {$[0.056]$} & {$[0.032]$} & {$[0.014]^{*}$} \\
\hline \multirow{2}{*}{$\tau_{w / c o v}^{C I C}$} & 0.112 & 0.114 & 0.462 & 0.050 & 0.091 & 0.093 \\
\hline & {$[0.129]$} & {$[0.298]$} & {$[0.747]$} & {$[0.059]$} & {$[0.067]$} & {$[0.062]$} \\
\hline \multicolumn{7}{|c|}{ Mujeres } \\
\hline \multirow[t]{2}{*}{$\tau^{C I C}$} & 0.043 & 0.000 & 0.000 & 0.000 & -0.069 & 0.000 \\
\hline & {$[0.156]$} & {$[0.000]$} & {$[0.000]$} & {$[0.122]$} & {$[0.056]$} & {$[0.047]$} \\
\hline \multirow{2}{*}{$\tau_{w / \operatorname{cov}}^{C I C}$} & -0.032 & -0.011 & -0.061 & -0.077 & -0.103 & -0.057 \\
\hline & {$[0.168]$} & {$[0.046]$} & {$[0.265]$} & {$[0.100]$} & {$[0.065]$} & {$[0.067]$} \\
\hline \multicolumn{7}{|c|}{ Hombres } \\
\hline$\tau^{C I C}$ & 0.147 & 0.357 & 0.000 & 0.000 & -0.080 & 0.000 \\
\hline \multirow{3}{*}{$\tau_{w / \operatorname{cov}}^{C I C}$} & {$[0.091]$} & {$[0.988]$} & {$[0.123]$} & {$[0.005]$} & {$[0.052]$} & {$[0.014]$} \\
\hline & 0.139 & 0.577 & -0.009 & 0.003 & 0.067 & 0.013 \\
\hline & {$[0.121]$} & {$[0.975]$} & {$[0.098]$} & {$[0.046]$} & {$[0.054]$} & {$[0.057]$} \\
\hline
\end{tabular}

Fuente: Estimación de los autores basada en la serie armonizada por Fundación ARU de encuestas de hogares (INE). 
Tabla 39: Efectos sobre las $(\log )$ horas trabajadas en la actividad primaria de futuros beneficiarios, estimador Changes-in-Changes

\begin{tabular}{|c|c|c|c|c|c|c|c|}
\hline \multirow{2}{*}{\multicolumn{2}{|c|}{ Bolivia }} & $C I C_{\text {promedio }}$ & \multirow{3}{*}{$\begin{array}{r}C I C_{q 0,1} \\
0.000\end{array}$} & \multirow{3}{*}{$\begin{array}{r}C I C_{q 0,2} \\
0.470\end{array}$} & \multirow{3}{*}{$\begin{array}{r}C I C_{q 0,5} \\
0.000\end{array}$} & \multirow{3}{*}{$\begin{array}{r}C I C_{q 0,8} \\
0.000\end{array}$} & \multirow{2}{*}{$C I C_{q 0,9}$} \\
\hline & & & & & & & \\
\hline$\tau^{C I C}$ & & 0.142 & & & & & 0.000 \\
\hline & & {$[0.082]^{*}$} & {$[0.000]$} & {$[0.931]$} & {$[0.049]$} & {$[0.026]$} & {$[0.007]$} \\
\hline \multirow{2}{*}{$\tau_{w / \mathrm{cov}}^{C I C}$} & & 0.128 & 0.114 & 0.405 & 0.034 & 0.092 & 0.119 \\
\hline & & {$[0.119]$} & {$[0.248]$} & {$[0.718]$} & {$[0.096]$} & {$[0.066]$} & {$[0.050]^{* *}$} \\
\hline \multirow{3}{*}{\multicolumn{2}{|c|}{$\tau^{\text {Mujeres }}$}} & & & & & & \\
\hline & & 0.055 & 0.000 & 0.000 & 0.000 & 0.000 & 0.000 \\
\hline & & {$[0.149]$} & {$[0.000]$} & {$[0.000]$} & {$[0.121]$} & {$[0.027]$} & {$[0.066]$} \\
\hline \multirow{2}{*}{$\tau_{w / \mathrm{cov}}^{C I C}$} & & -0.024 & -0.007 & -0.029 & -0.075 & -0.045 & -0.015 \\
\hline & & {$[0.155]$} & [0.043] & [0.209] & [0.091] & {$[0.085]$} & {$[0.071]$} \\
\hline \multirow{2}{*}{$\tau^{\text {Hol }}$} & bres & & & & & & \\
\hline & & 0.163 & 0.405 & 0.182 & 0.000 & 0.000 & -0.028 \\
\hline \multirow{3}{*}{$\tau_{w / \mathrm{cov}}^{C I C}$} & & {$[0.105]$} & {$[0.971]$} & [0.143] & {$[0.022]$} & {$[0.052]$} & {$[0.014]^{*}$} \\
\hline & & 0.172 & 0.662 & 0.021 & -0.033 & 0.110 & 0.027 \\
\hline & & {$[0.103]^{*}$} & {$[0.946]$} & {$[0.112]$} & {$[0.039]$} & {$[0.061]^{*}$} & {$[0.062]$} \\
\hline
\end{tabular}

Fuente: Estimación de los autores basada en la serie armonizada por Fundación ARU de encuestas de hogares (INE). 
Tabla 40: Efectos sobre el $(\log )$ salario por hora de futuros beneficiarios, estimador Changesin-Changes

\begin{tabular}{|c|c|c|c|c|c|c|}
\hline & $C I C_{\text {promedio }}$ & $C I C_{q 0,1}$ & $C I C_{q 0,2}$ & $C I C_{q 0,5}$ & $C I C_{q 0,8}$ & $C I C_{q 0,9}$ \\
\hline Boli & & & & & & \\
\hline$\tau^{C I C}$ & 0.554 & 0.000 & 0.000 & 0.396 & 0.157 & 0.112 \\
\hline & {$[0.199]^{* * *}$} & {$[0.000]$} & {$[0.000]$} & {$[0.185]^{* *}$} & {$[0.082]^{*}$} & {$[0.082]$} \\
\hline$\tau_{w / \operatorname{cov}}^{C I C}$ & 0.650 & 0.341 & 1.975 & 0.772 & 0.174 & 0.326 \\
\hline & {$[0.271]^{* *}$} & [0.239] & {$[0.638]^{* * *}$} & {$[0.317]^{* *}$} & {$[0.201]$} & [0.199] \\
\hline Muj & res & & & & & \\
\hline$\tau^{C I C}$ & 0.644 & 0.000 & 0.000 & 5.746 & 0.288 & 0.435 \\
\hline & {$[0.298]^{* *}$} & {$[0.000]$} & {$[0.000]$} & {$[1.671]^{* * *}$} & {$[0.161]^{*}$} & {$[0.183]^{* *}$} \\
\hline$\tau_{w / \operatorname{cov} v}^{C I C}$ & 0.663 & 0.031 & 0.254 & 3.345 & 0.445 & 0.732 \\
\hline & {$[0.317]^{* *}$} & {$[0.122]$} & {$[0.221]$} & {$[1.406]^{* *}$} & {$[0.304]$} & {$[0.344]^{* *}$} \\
\hline Hor & bres & & & & & \\
\hline$\tau^{C I C}$ & 0.258 & 0.642 & 0.604 & 0.112 & 0.092 & -0.000 \\
\hline & {$[0.190]$} & [1.608] & {$[0.306]^{* *}$} & {$[0.104]$} & {$[0.129]$} & {$[0.158]$} \\
\hline$\tau_{w / \operatorname{cov}}^{C I C}$ & 0.267 & 2.425 & 0.450 & 0.043 & 0.225 & 0.155 \\
\hline & {$[0.201]$} & {$[1.051]^{* *}$} & {$[0.504]$} & {$[0.200]$} & {$[0.164]$} & {$[0.142]$} \\
\hline
\end{tabular}

Fuente: Estimación de los autores basada en la serie armonizada por Fundación ARU de encuestas de hogares (INE). 
Tabla 41: Efectos sobre el (log) ingreso laboral mensual de futuros beneficiarios, estimador Changes-in-Changes

\begin{tabular}{|c|c|c|c|c|c|c|}
\hline & $C I C_{\text {promedio }}$ & $C I C_{q 0,1}$ & $C I C_{q 0,2}$ & $C I C_{q 0,5}$ & $C I C_{q 0,8}$ & $C I C_{q 0,9}$ \\
\hline \multicolumn{7}{|c|}{ Bolivia } \\
\hline \multirow[t]{2}{*}{$\tau^{C I C}$} & 0.520 & 0.000 & 0.000 & 0.266 & 0.046 & 0.056 \\
\hline & {$[0.225]^{* *}$} & {$[0.000]$} & {$[0.000]$} & {$[0.159]^{*}$} & {$[0.063]$} & {$[0.078]$} \\
\hline \multirow{2}{*}{$\tau_{w / c o v}^{C I C}$} & 0.633 & 0.427 & 3.198 & 0.710 & 0.124 & -0.155 \\
\hline & {$[0.282]^{* *}$} & {$[0.260]$} & {$[0.899]^{* * *}$} & {$[0.271]^{* * *}$} & {$[0.196]$} & {$[0.150]$} \\
\hline \multicolumn{7}{|c|}{ Mujeres } \\
\hline \multirow[t]{2}{*}{$\tau^{C I C}$} & 0.734 & 0.000 & 0.000 & 1.242 & 0.223 & 0.143 \\
\hline & {$[0.354]^{* *}$} & {$[0.000]$} & {$[0.000]$} & [1.995] & {$[0.178]$} & {$[0.159]$} \\
\hline \multirow{2}{*}{$\tau_{w / \operatorname{cov}}^{C I C}$} & 0.739 & 0.064 & 0.368 & 1.609 & 0.217 & 0.350 \\
\hline & {$[0.327]^{* *}$} & {$[0.109]$} & {$[0.252]$} & [1.661] & {$[0.267]$} & {$[0.242]$} \\
\hline \multicolumn{7}{|c|}{ Hombres } \\
\hline$\tau^{C I C}$ & 0.021 & 0.056 & 0.389 & -0.057 & -0.024 & -0.050 \\
\hline \multirow{3}{*}{$\tau_{w / \operatorname{cov}}^{C I C}$} & {$[0.216]$} & [1.889] & {$[0.304]$} & {$[0.101]$} & {$[0.068]$} & [0.103] \\
\hline & 0.033 & 0.443 & 0.217 & -0.029 & -0.211 & 0.032 \\
\hline & {$[0.230]$} & {$[1.432]$} & {$[0.484]$} & {$[0.178]$} & {$[0.143]$} & {$[0.168]$} \\
\hline
\end{tabular}

Fuente: Estimación de los autores basada en la serie armonizada por Fundación ARU de encuestas de hogares (INE). 
Tabla 42: Estimados de Diff-in-Diff de indicadores de nivel y sectores de participación de beneficiarios indirectos

\begin{tabular}{|c|c|c|c|c|c|c|c|c|c|}
\hline & \multicolumn{3}{|c|}{ Bolivia } & \multicolumn{3}{|c|}{ Hombres } & \multicolumn{3}{|c|}{ Mujeres } \\
\hline & $I C$ & $I T$ & DID & $I C$ & $I T$ & DID & $I C$ & $I T$ & DID \\
\hline \multicolumn{10}{|c|}{ Participación } \\
\hline \multirow[t]{2}{*}{$\mathrm{T}=0$} & 0.77 & 0.83 & & 0.86 & 0.90 & & 0.68 & 0.76 & \\
\hline & {$[0.02]$} & {$[0.02]$} & -0.04 & {$[0.02]$} & {$[0.02]$} & -0.02 & {$[0.03]$} & {$[0.03]$} & -0.04 \\
\hline \multirow[t]{2}{*}{$\mathrm{T}=1$} & 0.81 & 0.83 & {$[0.03]$} & 0.88 & 0.90 & {$[0.04]$} & 0.74 & 0.77 & [0.05] \\
\hline & {$[0.01]$} & {$[0.01]$} & & {$[0.02]$} & {$[0.02]$} & & {$[0.02]$} & {$[0.02]$} & \\
\hline \multicolumn{10}{|c|}{ Trabajador familiar } \\
\hline \multirow[t]{2}{*}{$\mathrm{T}=0$} & 0.15 & 0.18 & & 0.13 & 0.15 & & 0.16 & 0.21 & \\
\hline & {$[0.02]$} & {$[0.02]$} & 0.01 & {$[0.02]$} & {$[0.02]$} & 0.01 & {$[0.02]$} & {$[0.02]$} & -0.00 \\
\hline \multirow[t]{2}{*}{$\mathrm{T}=1$} & 0.13 & 0.17 & {$[0.03]$} & 0.10 & 0.13 & {$[0.04]$} & 0.17 & 0.21 & {$[0.04]$} \\
\hline & {$[0.01]$} & {$[0.02]$} & & {$[0.02]$} & {$[0.02]$} & & {$[0.02]$} & {$[0.02]$} & \\
\hline \multicolumn{10}{|c|}{ Informal n/asalariado } \\
\hline \multirow[t]{2}{*}{$\mathrm{T}=0$} & 0.19 & 0.22 & & 0.23 & 0.26 & & 0.15 & 0.18 & \\
\hline & {$[0.02]$} & {$[0.02]$} & 0.00 & {$[0.03]$} & {$[0.03]$} & 0.00 & {$[0.02]$} & {$[0.02]$} & 0.01 \\
\hline \multirow[t]{2}{*}{$\mathrm{T}=1$} & 0.18 & 0.21 & {$[0.03]$} & 0.21 & 0.24 & {$[0.05]$} & 0.15 & 0.19 & [0.04] \\
\hline & {$[0.01]$} & {$[0.02]$} & & {$[0.02]$} & {$[0.02]$} & & {$[0.02]$} & {$[0.02]$} & \\
\hline \multicolumn{10}{|c|}{ Informal asalariado } \\
\hline \multirow[t]{2}{*}{$\mathrm{T}=0$} & 0.25 & 0.21 & & 0.33 & 0.26 & & 0.16 & 0.17 & \\
\hline & {$[0.02]$} & {$[0.02]$} & -0.02 & {$[0.03]$} & {$[0.03]$} & 0.00 & {$[0.02]$} & {$[0.02]$} & -0.02 \\
\hline \multirow[t]{2}{*}{$\mathrm{T}=1$} & 0.26 & 0.21 & {$[0.04]$} & 0.33 & 0.26 & {$[0.05]$} & 0.19 & 0.18 & {$[0.04]$} \\
\hline & {$[0.02]$} & {$[0.02]$} & & {$[0.03]$} & {$[0.03]$} & & {$[0.02]$} & {$[0.02]$} & \\
\hline \multicolumn{10}{|c|}{ Formal asalariado } \\
\hline \multirow[t]{2}{*}{$\mathrm{T}=0$} & 0.13 & 0.16 & & 0.12 & 0.16 & & 0.13 & 0.15 & \\
\hline & {$[0.01]$} & {$[0.02]$} & -0.00 & {$[0.02]$} & {$[0.02]$} & 0.02 & {$[0.02]$} & {$[0.02]$} & -0.02 \\
\hline \multirow[t]{2}{*}{$\mathrm{T}=1$} & 0.18 & 0.20 & {$[0.03]$} & 0.18 & 0.24 & {$[0.04]$} & 0.18 & 0.18 & {$[0.04]$} \\
\hline & {$[0.01]$} & {$[0.02]$} & & {$[0.02]$} & {$[0.02]$} & & {$[0.02]$} & {$[0.02]$} & \\
\hline
\end{tabular}

Fuente: Estimación de los autores basada en la serie armonizada por Fundación ARU de encuestas de hogares (INE).

Notas: Errores estándar entre corchetes (ver nota de figura 7). IC=control indirecto son las personas en el intervalo $[25,44)$ de edad que viven con personas en el rango $[55,60)$, IT=tratamiento indirecto son las personas en el intervalo $[25,44)$ de edad que viven con personas en el rango $[60,65)$. 
Tabla 43: Efectos sobre la participación en el mercado laboral de beneficiarios indirectos, estimador Changes-in-Changes

\begin{tabular}{|c|c|c|c|}
\hline & $C I C_{\text {disc }}$ & $C I C_{d i s c} \mathrm{LB}$ & $C I C_{d i s c} \mathrm{UB}$ \\
\hline \multicolumn{4}{|c|}{ Bolivia } \\
\hline \multirow[t]{2}{*}{$\tau^{C I C}$} & -0.03 & -0.17 & 0.01 \\
\hline & {$[0.030]$} & {$[0.053]^{* * *}$} & {$[0.221]$} \\
\hline \multirow{2}{*}{$\tau_{w / \operatorname{cov}}^{C I C}$} & -0.03 & -0.03 & -0.03 \\
\hline & [0.028] & {$[0.028]$} & [0.028] \\
\hline \multicolumn{4}{|c|}{ Mujeres } \\
\hline \multirow[t]{2}{*}{$\tau^{C I C}$} & -0.03 & -0.23 & 0.02 \\
\hline & {$[0.042]$} & {$[0.068]^{* * *}$} & [0.209] \\
\hline \multirow{2}{*}{$\tau_{w / \operatorname{cov}}^{C I C}$} & -0.03 & -0.03 & -0.03 \\
\hline & {$[0.052]$} & {$[0.052]$} & {$[0.052]$} \\
\hline \multicolumn{4}{|c|}{ Hombres } \\
\hline$\tau^{C I C}$ & -0.02 & -0.10 & 0.00 \\
\hline \multirow{3}{*}{$\tau_{w / \operatorname{cov}}^{C I C}$} & {$[0.032]$} & {$[0.046]^{* *}$} & {$[0.245]$} \\
\hline & -0.01 & -0.01 & -0.01 \\
\hline & {$[0.030]$} & {$[0.030]$} & {$[0.030]$} \\
\hline
\end{tabular}

Fuente: Estimación de los autores basada en la serie armonizada por Fundación ARU de encuestas de hogares (INE). $\mathrm{LB}=$ límite inferior (lower bound), UB=límite superior (upper bound). 
Tabla 44: Efectos sobre los sectores de participación laboral de beneficiarios indirectos, estimador Changes-in-Changes

\section{(a) Trabajador familiar}

\begin{tabular}{|c|c|c|c|}
\hline & $C I C_{d i s c}$ & $C I C_{d i s c} \mathrm{LB}$ & $C I C_{d i s c} \mathrm{UB}$ \\
\hline \multicolumn{4}{|c|}{ Bolivia } \\
\hline \multirow[t]{2}{*}{$\tau^{C I C}$} & 0.01 & -0.01 & 0.17 \\
\hline & [0.030] & {$[0.228]$} & {$[0.063]^{* * *}$} \\
\hline \multirow{2}{*}{$\tau_{w / c o v}^{C I C}$} & 0.01 & 0.01 & 0.01 \\
\hline & [0.044] & {$[0.044]$} & {$[0.044]$} \\
\hline \multicolumn{4}{|c|}{ Mujeres } \\
\hline \multirow{2}{*}{$\tau^{C I C}$} & -0.00 & -0.79 & -0.00 \\
\hline & [0.041] & {$[0.228]^{* * * *} *$} & {$[0.072]$} \\
\hline \multirow{2}{*}{$\tau_{w / c o v}^{C I C}$} & -0.02 & -0.02 & -0.02 \\
\hline & [0.071] & {$[0.071]$} & {$[0.071]$} \\
\hline \multicolumn{4}{|c|}{ Hombres } \\
\hline \multirow[t]{2}{*}{$\tau^{C I C}$} & 0.01 & -0.03 & 0.13 \\
\hline & {$[0.034]$} & {$[0.227]$} & {$[0.050]^{* *}$} \\
\hline \multirow{2}{*}{$\tau_{w / c o v}^{C I C}$} & 0.04 & 0.04 & 0.04 \\
\hline & {$[0.061]$} & {$[0.061]$} & {$[0.061]$} \\
\hline
\end{tabular}

\section{(c) Informal asalariado}

\begin{tabular}{|c|c|c|c|}
\hline & $C I C_{d i s c}$ & $C I C_{d i s c} \mathrm{LB}$ & $C I C_{d i s c} \mathrm{UB}$ \\
\hline \multicolumn{4}{|c|}{ Bolivia } \\
\hline \multirow{2}{*}{$\tau^{C I C}$} & -0.02 & -0.79 & 0.00 \\
\hline & {$[0.038]$} & {$[0.218]^{* * *}$} & {$[0.068]$} \\
\hline \multirow[t]{2}{*}{$\tau_{w / c o v}^{C I C}$} & -0.02 & -0.02 & -0.02 \\
\hline & {$[0.036]$} & {$[0.036]$} & {$[0.036]$} \\
\hline \multicolumn{4}{|c|}{ Mujeres } \\
\hline \multirow[t]{2}{*}{$\tau^{C I C}$} & -0.02 & -0.82 & 0.00 \\
\hline & {$[0.041]$} & {$[0.229]^{* * *}$} & {$[0.065]$} \\
\hline \multirow[t]{2}{*}{$\tau_{w / c o v}^{C I C}$} & -0.02 & -0.02 & -0.02 \\
\hline & {$[0.047]$} & {$[0.047]$} & {$[0.047]$} \\
\hline \multicolumn{4}{|c|}{ Hombres } \\
\hline \multirow[t]{2}{*}{$\tau^{C I C}$} & 0.00 & -0.00 & 0.26 \\
\hline & {$[0.047]$} & {$[0.216]$} & {$[0.091] * * *$} \\
\hline \multirow{2}{*}{$\tau_{w / c o v}^{C I C}$} & -0.01 & -0.01 & -0.01 \\
\hline & {$[0.060]$} & {$[0.060]$} & {$[0.059]$} \\
\hline
\end{tabular}

\section{(b) Informal no asalariado}

\begin{tabular}{|c|c|c|c|}
\hline & $C I C_{\text {disc }}$ & $C I C_{d i s c} \mathrm{LB}$ & $C I C_{d i s c}$ UB \\
\hline \multicolumn{4}{|c|}{ Bolivia } \\
\hline \multirow[t]{2}{*}{$\tau^{C I C}$} & 0.01 & -0.01 & 0.21 \\
\hline & [0.035] & {$[0.216]$} & {$[0.072] * * *$} \\
\hline \multirow{2}{*}{$\tau_{w / c o v}^{C I C}$} & 0.01 & 0.01 & 0.01 \\
\hline & [0.044] & {$[0.044]$} & [0.044] \\
\hline \multicolumn{4}{|c|}{ Mujeres } \\
\hline \multirow[t]{2}{*}{$\tau^{C I C}$} & 0.01 & 0.01 & 0.19 \\
\hline & {$[0.049]$} & {$[0.229]$} & {$[0.075]^{* *}$} \\
\hline \multirow{2}{*}{$\tau_{w / c o v}^{C I C}$} & 0.03 & 0.03 & 0.03 \\
\hline & {$[0.050]$} & {$[0.050]$} & {$[0.050]$} \\
\hline \multicolumn{4}{|c|}{ Hombres } \\
\hline \multirow[t]{2}{*}{$\tau^{C I C}$} & 0.01 & -0.02 & 0.24 \\
\hline & {$[0.058]$} & {$[0.218]$} & {$[0.099]^{* *}$} \\
\hline \multirow{2}{*}{$\tau_{w / c o v}^{C I C}$} & 0.01 & 0.01 & 0.01 \\
\hline & {$[0.055]$} & {$[0.055]$} & {$[0.055]$} \\
\hline
\end{tabular}

(d) Formal asalariado

\begin{tabular}{|c|c|c|c|}
\hline & $C I C_{\text {disc }}$ & $C I C_{d i s c} \mathrm{LB}$ & $C I C_{d i s c} \mathrm{UB}$ \\
\hline \multicolumn{4}{|c|}{ Bolivia } \\
\hline \multirow[t]{2}{*}{$\tau^{C I C}$} & -0.00 & -0.80 & 0.05 \\
\hline & {$[0.038]$} & {$[0.020]^{* * *}$} & {$[0.033]$} \\
\hline \multirow{2}{*}{$\tau_{w / c o v}^{C I C}$} & -0.01 & -0.01 & -0.01 \\
\hline & [0.034] & {$[0.035]$} & {$[0.034]$} \\
\hline \multicolumn{4}{|c|}{ Mujeres } \\
\hline \multirow[t]{2}{*}{$\tau^{C I C}$} & -0.02 & -0.82 & 0.02 \\
\hline & {$[0.042]$} & {$[0.222]^{* * *}$} & {$[0.050]$} \\
\hline \multirow[t]{2}{*}{$\tau_{w / \text { cov }}^{C I C}$} & -0.03 & -0.03 & -0.03 \\
\hline & [0.056] & {$[0.058]$} & {$[0.056]$} \\
\hline \multicolumn{4}{|c|}{ Hombres } \\
\hline \multirow[t]{2}{*}{$\tau^{C I C}$} & 0.02 & -0.76 & 0.07 \\
\hline & [0.045] & {$[0.022]^{* * *}$} & {$[0.033]^{* *}$} \\
\hline \multirow[t]{2}{*}{$\tau_{w / \text { cov }}^{C I C}$} & 0.01 & 0.01 & 0.01 \\
\hline & [0.048] & {$[0.049]$} & {$[0.048]$} \\
\hline
\end{tabular}

$* * * \mathrm{p}<0.01, * * \mathrm{p}<0.05, * \mathrm{p}<0.1$

Fuente: Estimación de los autores basada en la serie armonizada por Fundación ARU de encuestas de hogares (INE). $\mathrm{LB}=$ límite inferior (lower bound), $\mathrm{UB}=$ límite superior (upper bound). 
Tabla 45: Estimados de Diff-in-Diff de las horas trabajadas, salario por hora, e ingresos laborales mensuales de beneficiarios indirectos

\begin{tabular}{|c|c|c|c|c|c|c|c|c|c|}
\hline & \multicolumn{3}{|c|}{ Bolivia } & \multicolumn{3}{|c|}{ Hombres } & \multicolumn{3}{|c|}{ Mujeres } \\
\hline & $I C$ & $I T$ & DID & $I C$ & $I T$ & DID & $I C$ & $I T$ & DID \\
\hline \multicolumn{10}{|c|}{ Intensidad de oferta total (log de horas por mes) } \\
\hline \multirow[t]{2}{*}{$\mathrm{T}=0$} & 2.39 & 2.72 & & 2.89 & 3.03 & & 1.90 & 2.44 & \\
\hline & {$[0.09]$} & {$[0.09]$} & -0.18 & {$[0.11]$} & {$[0.11]$} & 0.02 & {$[0.13]$} & {$[0.12]$} & -0.31 \\
\hline \multirow[t]{2}{*}{$\mathrm{T}=1$} & 2.65 & 2.81 & {$[0.16]$} & 2.99 & 3.16 & {$[0.21]$} & 2.27 & 2.50 & {$[0.24]$} \\
\hline & {$[0.08]$} & {$[0.07]$} & & {$[0.09]$} & {$[0.10]$} & & {$[0.12]$} & {$[0.11]$} & \\
\hline \multicolumn{10}{|c|}{ Intensidad de oferta en la actividad primaria (log de horas por mes) } \\
\hline \multirow[t]{2}{*}{$\mathrm{T}=0$} & 2.37 & 2.70 & & 2.88 & 3.00 & & 1.88 & 2.41 & \\
\hline & {$[0.09]$} & {$[0.08]$} & -0.18 & {$[0.11]$} & {$[0.11]$} & 0.02 & {$[0.13]$} & {$[0.12]$} & -0.30 \\
\hline \multirow[t]{2}{*}{$\mathrm{T}=1$} & 2.63 & 2.78 & {$[0.16]$} & 2.97 & 3.11 & {$[0.21]$} & 2.25 & 2.49 & {$[0.24]$} \\
\hline & {$[0.08]$} & {$[0.07]$} & & {$[0.09]$} & {$[0.09]$} & & {$[0.12]$} & {$[0.11]$} & \\
\hline \multicolumn{10}{|c|}{ Salario (log de Bs. 2012 por hora) } \\
\hline \multirow[t]{2}{*}{$\mathrm{T}=0$} & -1.57 & -1.25 & & -0.66 & -0.42 & & -2.44 & -2.01 & \\
\hline & {$[0.18]$} & {$[0.18]$} & -0.37 & {$[0.24]$} & {$[0.25]$} & -0.27 & {$[0.24]$} & {$[0.25]$} & -0.31 \\
\hline \multirow[t]{2}{*}{$\mathrm{T}=1$} & -0.87 & -0.92 & {$[0.33]$} & -0.09 & -0.12 & {$[0.45]$} & -1.73 & -1.61 & {$[0.47]$} \\
\hline & {$[0.15]$} & {$[0.16]$} & & {$[0.19]$} & {$[0.22]$} & & {$[0.23]$} & {$[0.23]$} & \\
\hline \multicolumn{10}{|c|}{ Ingreso laboral mensual (log de Bs. 2012 por mes) } \\
\hline \multirow[t]{2}{*}{$\mathrm{T}=0$} & 3.08 & 3.47 & & 4.14 & 4.45 & & 2.07 & 2.57 & \\
\hline & {$[0.20]$} & {$[0.21]$} & -0.50 & {$[0.27]$} & {$[0.29]$} & -0.42 & {$[0.28]$} & {$[0.28]$} & -0.37 \\
\hline \multirow[t]{2}{*}{$\mathrm{T}=1$} & 3.92 & 3.81 & {$[0.38]$} & 4.90 & 4.80 & {$[0.52]$} & 2.83 & 2.96 & {$[0.54]$} \\
\hline & {$[0.17]$} & {$[0.19]$} & & {$[0.22]$} & {$[0.26]$} & & {$[0.26]$} & {$[0.26]$} & \\
\hline
\end{tabular}

Fuente: Estimación de los autores basada en la serie armonizada por Fundación ARU de encuestas de hogares (INE).

Notas: Errores estándar entre corchetes (ver nota de figura 7). IC=control indirecto son las personas en el intervalo $[25,44)$ de edad que viven con personas en el rango $[55,60)$, IT=tratamiento indirecto son las personas en el intervalo $[25,44)$ de edad que viven con personas en el rango $[60,65)$. 
Tabla 46: Efectos sobre las (log) horas trabajadas en total de los beneficiarios indirectos, estimador Changes-in-Changes

\begin{tabular}{|c|c|c|c|c|c|c|}
\hline & $C I C_{\text {promedio }}$ & $C I C_{q 0,1}$ & $C I C_{q 0,2}$ & $C I C_{q 0,5}$ & $C I C_{q 0,8}$ & $C I C_{q 0,9}$ \\
\hline \multicolumn{7}{|c|}{ Bolivia } \\
\hline \multirow[t]{2}{*}{$\tau^{C I C}$} & -0.133 & 0.000 & -2.079 & -0.095 & -0.069 & -0.134 \\
\hline & {$[0.134]$} & {$[0.000]$} & {$[1.798]$} & {$[0.047]^{* *}$} & {$[0.027]^{* *}$} & {$[0.075]^{*}$} \\
\hline \multirow[t]{2}{*}{$\tau_{w / \operatorname{cov}}^{C I C}$} & -0.198 & -0.035 & -0.236 & -0.231 & -0.091 & -0.162 \\
\hline & {$[0.176]$} & {$[0.200]$} & {$[1.330]$} & {$[0.124]^{*}$} & {$[0.078]$} & {$[0.124]$} \\
\hline \multicolumn{7}{|c|}{ Mujeres } \\
\hline \multirow[t]{2}{*}{$\tau^{C I C}$} & -0.234 & 0.000 & 0.000 & -0.134 & -0.163 & -0.154 \\
\hline & {$[0.153]$} & {$[0.000]$} & {$[0.884]$} & {$[0.098]$} & {$[0.091]^{*}$} & {$[0.103]$} \\
\hline \multirow{2}{*}{$\tau_{w / \operatorname{cov}}^{C I C}$} & -0.284 & -0.031 & 0.061 & -0.384 & -0.041 & -0.024 \\
\hline & {$[0.179]$} & {$[0.149]$} & {$[1.083]$} & {$[0.125]^{* * *}$} & {$[0.158]$} & {$[0.130]$} \\
\hline \multicolumn{7}{|c|}{ Hombres } \\
\hline \multirow[t]{2}{*}{$\tau^{C I C}$} & 0.044 & 0.000 & -0.182 & 0.000 & -0.069 & -0.028 \\
\hline & {$[0.190]$} & {$[0.000]$} & {$[1.255]$} & {$[0.039]$} & {$[0.057]$} & {$[0.086]$} \\
\hline \multirow{2}{*}{$\tau_{w / c o v}^{C I C}$} & 0.034 & 0.269 & 0.067 & -0.052 & -0.122 & -0.168 \\
\hline & {$[0.219]$} & {$[0.310]$} & {$[1.209]$} & {$[0.115]$} & {$[0.160]$} & [0.133] \\
\hline
\end{tabular}

Fuente: Estimación de los autores basada en la serie armonizada por Fundación ARU de encuestas de hogares (INE). 
Tabla 47: Efectos sobre las (log) horas trabajadas en la actividad primaria de beneficiarios indirectos, estimador Changes-in-Changes

\begin{tabular}{|c|c|c|c|c|c|c|}
\hline & CIC $C_{\text {promedio }}$ & $C I C_{q 0,1}$ & $C I C_{q 0,2}$ & $C I C_{q 0,5}$ & $C I C_{q 0,8}$ & $C I C_{q 0,9}$ \\
\hline \multicolumn{7}{|c|}{ Bolivia } \\
\hline \multirow[t]{2}{*}{$\tau^{C I C}$} & -0.132 & 0.000 & -1.386 & -0.095 & -0.036 & -0.154 \\
\hline & {$[0.138]$} & {$[0.000]$} & [1.798] & {$[0.047]^{* *}$} & {$[0.047]$} & {$[0.050]^{* * *}$} \\
\hline \multirow[t]{2}{*}{$\tau_{w / c o v}^{C I C}$} & -0.196 & -0.015 & -0.106 & -0.208 & -0.159 & -0.201 \\
\hline & {$[0.144]$} & {$[0.155]$} & {$[1.231]$} & {$[0.107]^{*}$} & {$[0.077]^{* *}$} & {$[0.123]$} \\
\hline \multicolumn{7}{|c|}{ Mujeres } \\
\hline \multirow[t]{2}{*}{$\tau^{C I C}$} & -0.229 & 0.000 & 0.000 & -0.288 & -0.113 & -0.154 \\
\hline & {$[0.251]$} & {$[0.000]$} & {$[1.091]$} & {$[0.120]^{* *}$} & {$[0.076]$} & [0.103] \\
\hline \multirow[t]{2}{*}{$\tau_{w / \operatorname{cov}}^{C I C}$} & -0.281 & -0.070 & 0.028 & -0.379 & -0.097 & -0.004 \\
\hline & {$[0.205]$} & {$[0.167]$} & [1.034] & {$[0.170]^{* *}$} & {$[0.143]$} & [0.149] \\
\hline \multicolumn{7}{|c|}{ Hombres } \\
\hline \multirow[t]{2}{*}{$\tau^{C I C}$} & 0.032 & 0.000 & -0.405 & 0.000 & 0.000 & -0.087 \\
\hline & {$[0.183]$} & {$[0.811]$} & {$[1.248]$} & {$[0.069]$} & {$[0.066]$} & [0.106] \\
\hline \multirow[t]{2}{*}{$\tau_{w / \operatorname{cov}}^{C I C}$} & 0.032 & 0.292 & -0.017 & -0.066 & -0.055 & -0.063 \\
\hline & {$[0.172]$} & {$[0.268]$} & {$[1.145]$} & {$[0.111]$} & {$[0.116]$} & {$[0.115]$} \\
\hline
\end{tabular}

Fuente: Estimación de los autores basada en la serie armonizada por Fundación ARU de encuestas de hogares (INE). 
Tabla 48: Efectos sobre el $(\log )$ salario por hora de beneficiarios indirectos, estimador Changes-in-Changes

\begin{tabular}{|c|c|c|c|c|c|c|}
\hline & $C I C_{\text {promedio }}$ & $C I C_{q 0,1}$ & $C I C_{q 0,2}$ & $C I C_{q 0,5}$ & $C I C_{q 0,8}$ & $C I C_{q 0,9}$ \\
\hline \multicolumn{7}{|c|}{ Bolivia } \\
\hline \multirow[t]{2}{*}{$\tau^{C I C}$} & -0.337 & 0.000 & 0.000 & -0.285 & -0.088 & -0.023 \\
\hline & {$[0.311]$} & {$[0.000]$} & {$[0.000]$} & {$[0.189]$} & {$[0.125]$} & [0.113] \\
\hline \multirow[t]{2}{*}{$\tau_{w / \operatorname{cov}}^{C I C}$} & -0.425 & -0.113 & -0.359 & -0.259 & -0.287 & -0.258 \\
\hline & {$[0.347]$} & {$[0.572]$} & {$[0.437]$} & {$[0.510]$} & {$[0.262]$} & {$[0.209]$} \\
\hline \multicolumn{7}{|c|}{ Mujeres } \\
\hline \multirow[t]{2}{*}{$\tau^{C I C}$} & -0.291 & 0.000 & 0.000 & -0.582 & -0.079 & 0.150 \\
\hline & {$[0.520]$} & {$[0.000]$} & {$[0.000]$} & {$[2.413]$} & {$[0.233]$} & [0.191] \\
\hline \multirow{2}{*}{$\tau_{w / \operatorname{cov}}^{C I C}$} & -0.054 & 0.123 & -0.164 & -0.626 & -0.440 & -0.218 \\
\hline & {$[0.489]$} & {$[0.440]$} & {$[0.542]$} & {$[2.386]$} & {$[0.289]$} & {$[0.315]$} \\
\hline \multicolumn{7}{|c|}{ Hombres } \\
\hline \multirow[t]{2}{*}{$\tau^{C I C}$} & -0.223 & 0.000 & 0.000 & -0.096 & -0.162 & -0.022 \\
\hline & {$[0.417]$} & {$[0.000]$} & {$[1.478]$} & {$[0.222]$} & {$[0.121]$} & {$[0.140]$} \\
\hline \multirow[t]{2}{*}{$\tau_{w / \operatorname{cov}}^{C I C}$} & -0.417 & -1.321 & -0.324 & 0.211 & -0.102 & -0.211 \\
\hline & {$[0.457]$} & {$[0.790]^{*}$} & {$[0.999]$} & {$[0.423]$} & {$[0.224]$} & {$[0.215]$} \\
\hline
\end{tabular}

Fuente: Estimación de los autores basada en la serie armonizada por Fundación ARU de encuestas de hogares (INE). 
Tabla 49: Efectos sobre el (log) ingreso laboral mensual de beneficiarios indirectos, estimador Changes-in-Changes

\begin{tabular}{|c|c|c|c|c|c|c|}
\hline & $C I C_{\text {promedio }}$ & $C I C_{q 0,1}$ & $C I C_{q 0,2}$ & $C I C_{q 0,5}$ & $C I C_{q 0,8}$ & $C I C_{q 0,9}$ \\
\hline \multicolumn{7}{|c|}{ Bolivia } \\
\hline \multirow[t]{2}{*}{$\tau^{C I C}$} & -0.425 & 0.000 & 0.000 & -0.355 & -0.153 & -0.164 \\
\hline & {$[0.345]$} & {$[0.000]$} & {$[0.000]$} & {$[0.166]^{* *}$} & {$[0.079]^{*}$} & {$[0.100]^{*}$} \\
\hline \multirow{2}{*}{$\tau_{w / c o v}^{C I C}$} & -0.551 & -0.058 & -0.515 & -0.440 & -0.353 & -0.222 \\
\hline & {$[0.416]$} & {$[0.789]$} & {$[0.528]$} & {$[0.481]$} & {$[0.310]$} & {$[0.272]$} \\
\hline \multicolumn{7}{|c|}{ Mujeres } \\
\hline \multirow[t]{2}{*}{$\tau^{C I C}$} & -0.314 & 0.000 & 0.000 & -0.877 & -0.098 & 0.091 \\
\hline & {$[0.503]$} & {$[0.000]$} & {$[0.000]$} & {$[4.287]$} & {$[0.155]$} & [0.159] \\
\hline \multirow[t]{2}{*}{$\tau_{w / \operatorname{cov}}^{C I C}$} & -0.110 & -0.072 & -0.080 & -0.343 & -0.572 & -0.193 \\
\hline & {$[0.559]$} & {$[0.470]$} & {$[0.585]$} & {$[2.842]$} & {$[0.297]^{*}$} & {$[0.343]$} \\
\hline \multicolumn{7}{|c|}{ Hombres } \\
\hline \multirow[t]{2}{*}{$\tau^{C I C}$} & -0.329 & 0.000 & 0.000 & -0.206 & -0.238 & -0.110 \\
\hline & {$[0.437]$} & {$[0.000]$} & [1.916] & {$[0.159]$} & {$[0.130]^{*}$} & [0.154] \\
\hline \multirow[t]{2}{*}{$\tau_{w / c o v}^{C I C}$} & -0.536 & -1.473 & 0.319 & -0.096 & -0.183 & -0.454 \\
\hline & {$[0.476]$} & {$[0.597]^{* *}$} & {$[0.559]$} & {$[0.442]$} & {$[0.250]$} & {$[0.237]^{*}$} \\
\hline
\end{tabular}

Fuente: Estimación de los autores basada en la serie armonizada por Fundación ARU de encuestas de hogares (INE). 


\section{A Datos y definiciones de variables}

A lo largo del documento trabajamos con variables en logaritmos y para no perder observaciones debido a los valores de cero en determinadas variables, recodificamos el cero con la mitad del mínimo valor observado (no cero). Véase por ejemplo Meyer, Viscusi, and Durbin (1995, pp330).

\section{A.1 Bienestar, inversiones y ahorro}

Aproximamos el bienestar a través del ingreso y consumo total del hogar per-cápita.

Ingreso Definimos el ingreso del hogar per-cápita disponible como la suma del ingreso laboral de la actividad primaria y secundaria, el ingreso por transferencias del gobierno (renta dignidad), el ingreso por propiedad, el ingreso por seguridad social, el ingreso por transferencias de otros hogares y otras fuentes de ingreso. El ingreso laboral ya excluye los impuesto de ley ergo es el ingreso disponible. El ingreso total es dividido por el número de miembros del hogar para obtener el equivalente per-cápita, se encuentra expresado en términos mensuales y es deflactado con el índice de precios al consumidor base diciembre 2012.

Consumo Definimos el consumo total del hogar como la suma de los bienes alimentarios consumidos dentro del hogar (compras, donaciones y auto-consumo) y fuera del hogar, el consumo de bienes no alimentarios (periódicos, transporte, tabaco, revistas, etc.), el consumo de servicios básicos (agua, luz, combustible para cocinar), el alquiler estimado de la vivienda.10 El consumo se expresa en valores mensuales y es deflactado por el número de miembros del hogar y con el índice de precios al consumidor base diciembre 2012.

Educación El gasto en educación se mide como el gasto total en pensiones y matrículas, gasto en uniforme escolar, material de escritorio, transporte a la escuela y otros (contribuciones a la junta de padres, recreo, etc.).

Salud El gasto en salud se mide como el gasto en consultas médicas, medicinas, servicios de internación médica y otros.

Adquisición de durables El gasto en bienes durables se mide como el gasto en adquisición durante el último año en bienes durables. Específicamente: juego de living, cocina, refrigerador, moto, auto, cama, ropero, juego de comedor, reproductor de video, máquina de coser, bicicleta, horno a gas, microondas, televisor, computadora, radiograbador, juegos de video, minicomponente, aire acondicionado, ventilador, estufa, lavadora y secadora.

Inversión en vivienda Esta inversión se mide como el gasto en reparación de techos, paredes, pisos, servicios sanitario, tuberías, sistema eléctrico del hogar y otros. Además se

10 Los resultados son similares si incluimos el consumo de bienes duraderos, educación y salud y se encuentren disponibles de los autores a petición. 
incluyen los gastos en construcción y ampliación de cuartos, construcción de cercas y muros, colocación de machimbre, colocación de cortinas y otros.

\section{A.2 Indicadores del mercado laboral}

Participación Es un indicador dicótomo que indica 1 si la participa o no en el mercado laboral (está empleada o desempleada) o no.

Horas trabajadas, intensidad de oferta Son las horas dedicadas al trabajo ya sea en todos las actividades o sola en la actividad primaria.

Sectores de empleo Definimos cuatro sectores de empleo: trabajadores familiares no remunerados, trabajadores informales no asalariados (i.e., cuenta propia con o sin empleados a cargo), trabajadores informales asalariados y trabajadores formales asalariados. Definimos el estatus de formalidad en términos de si el trabajador contribuye o no al sistema de pensión de largo plazo (AFP).

\section{B Extensiones del modelo básico de Cambios-en-Cambios}

Es posible incluir covariables en las estimaciones del modelo de CIC, que en el caso de variables discretas ayuda a cerrar los límites y mejorar la estimación puntual. El procedimiento es el siguiente:

1. Seã $Y_{g t, i}=Y_{g t, i}{ }^{-}{ }^{\prime}{ }_{g t, i}{ }^{\beta} \quad$ y $D=((1-T)(1-G), T(1-G),(1-T) G, T G)^{\prime}$

2. Estimar la regresión (MCO, sin constante) $Y_{i}=D^{\prime} \delta+X_{i}^{\prime} \beta+\varepsilon_{i}$

3. Obtener los residuos aumentados $\hat{Y}=Y_{i}-X_{i}^{\prime} \beta=D^{\prime} \delta+\varepsilon_{i}$, y aplicarles el estimador de CIC.

En el documento incluimos las siguientes covariables cuando así se indica:

1. Controles individuales

- Logro educativo (años de educación)

- Sexo (para la muestra de Bolivia)

- Etnicidad, medida a partir de un índice multidimensional que se basa en tres indicadores: i) habla o no una lengua indígena, ii) declaración de autopertenencia, y iii) si habla o no una lengua indígena. A partir de estos 3 indicadores se tienen las categorías no indígenas (si no responde positivamente a ningún indicador), mestizo (si responde positivamente a uno $o$ dos indicadores) e indígena (si responde positivamente a los 3 indicadores). 
- Variables dummy si hay individuos en el hogar en los siguientes intervalos de edad: $[0,3],[4,6],[7,15],[16,18]$

- Quintiles del índice de riqueza (véase Rutstein and Johnson (2004))

2. Otros controles

- Indicador para el área rural

- Indicadores de efectos fijos para los departamentos (consideramos a Pando y Beni un sólo departamento)

En el caso de variables del hogar utilizamos los datos correspondientes del jefe del hogar. 